
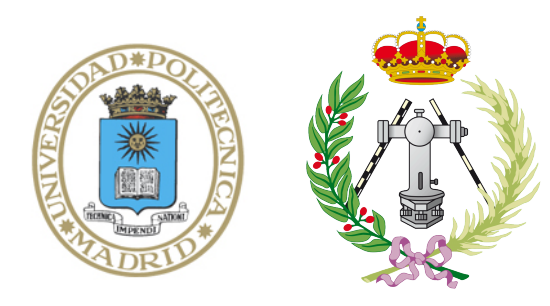

UNIVERSIDAD POLITÉCNICA DE MADRID

Escuela Técnica Superior de Ingenieros en

Topografía, Geodesia y Cartografía

\title{
Analysis of earthquake sequences and activity rates: implications for seismic hazard.
}

PhD Dissertation

POUYE YAZDI

MSc. in Geophysics

Advisors:

Dr. Jorge Miguel Gaspar Escribano

Dr. Miguel Ángel Santoyo García Galiano 

Tribunal nombrado por el Magfco. Y Excmo. Sr. Rector de la Universidad Politécnica de Madrid, el día. de. de 2020 .

Presidente D.

Vocal D.

Vocal D.

Vocal D.

Secretario D

Realizado el acto de defensa y lectura de la tesis el día. de. de 2020 .

Calificación:

EL PRESIDENTE LOS VOCALES

EL SECRETARIO 

She is complete, but never finished As she works now, so can she always work

Everyone sees her in his own fashion

She hides under a thousand names and phrases, and is always the same

She has brought me here and will also lead me away

I trust her. She may scold me, but she will not hate her work

It was not I who spoke of her. No!

What is false and what is true, she has spoken it all

The fault, the merit, is all hers

Aphorisms on NAture by Goethe (Huxley, 1869) 
TO MY PARENTS, AND TO MEHDI 


\section{Acknowledgements}

First and foremost, I wish to express my sincere gratitude to Dr. Jorge Gaspar-Escribano. Undeniably, without his invaluable guidance, this work would never have been made possible. I have been fortunate to be his student from the first year of my PhD program, have been introduced to the topic of main research in his class and benefited from his expertise during these years. I am profoundly indebted to his precious advise, his patience and his humane mentorship.

My utmost appreciation to Dr. Miguel Ángel Santoyo, from whom not only have I learned how to look into the earthquake phenomenon but he has also intrigued me with his integrity as a righteous and humble researcher always and specifically during my two months stay at Geophysics Institute of National Autonomous University of Mexico, Morelia, Mexico. I would like to thank him for generously providing me with the data in the fifth and sixth chapters of this dissertation.

I extend my sincere thanks to Dr. Sebastian Hainzl, who also generously provided me with the ETAS code I applied in third, fourth and sixth chapters of this dissertation, and for his very kind support and inspiring collaboration and co-authorship during my three months stay at German Geoscience Research Centre, Potsdam, Germany.

I am thankful to all professors at UPM from whom I learned the essential knowledge for conducting my research, and benefited from their influential feedback on my works. I would like to mention in particular, Dr. Bélen Benito, Dr. Alejandra Staller, Dr. José Luis García Pallero and Dr. Sandra Martínez.

I would like to thank Dr. Mehdi Zare and Dr. Allen Husker for kindly accepting to review this dissertation and for their time and invaluable advice.

During the doctoral program I enjoyed the companionship of close friends, among other, I would like to name Yolanda with whom I shared moments of trust and comfort, and also Ligia and Laura for all those distressing conversations and carefree laughter. More specially, I thank dearest Diego, for uncountable moments of joy and memories, his loyal friendship and emotional support.

Last but not least, my immeasurable gratitude to my parents for being an absolute shelter and believing in me. And my infinite gratefulness to Mehdi, for unconditional love, unfailing presence, pure friendship and cordiality, and most of all, never stop motivating me. 
THIS DOCUMENT IS EDITED IN LTTE 


\section{Abstract}

Earthquakes are natural phenomena that release enormous amounts of energy travelling in all directions and cause ground shaking when they reach to the Earth's surface. To mitigate their adverse effects on human lives and the environment, geophysicists investigate their causes. This involves studying earthquakes, not as isolated phenomena, but in relation and interaction with a series of earthquakes that share same time and/or space windows, and display common or similar features. This dissertation deals with the study of such series or earthquake sequences. I consider two different methodologies: statistical seismology, and physics-based methods.

The statistical approach considers earthquakes as objects with determined attributes: origin time, hypocentral coordinates and magnitude, which are available in earthquake catalogs. These attributes are treated as random values that have some probability distributions. Thus, the quality of earthquake catalogs directly affects the performance and validity of statistical models, which describe the probability distribution of the earthquake attributes. Here, I apply several statistical models in finite spatiotemporal windows and examine their application in individual or combined manners. For instance, the earthquake magnitude-frequency relation is modelled using the Gutenberg-Richter law, and for the analysing the temporal variation of earthquake occurrence, the Omori-Utsu and epidemic type aftershock sequence (ETAS) models are applied. The ETAS model is a point process in which each earthquake represents a point with the mentioned attributes. This model assumes that all the earthquakes can potentially trigger aftershocks, and display a branching process. Applying the ETAS model provides separate estimates of background and aftershock rates, which are associated with two distinct source-types, respectively: (1) aseismic sources such as tectonic loading, rapid fluid or magma intrusions and transient slow-slip events, and (2) seismic source (i.e., earthquake-earthquake elastic triggering) which controls the branching ratio. The respective contributions of seismic and aseismic sources, and their spatiotemporal variations are strongly determinative in our understanding about the nature of earthquake sequences. Overall, the result from statistical modelling indicates the nature of the branching processes via quantitative parameters. Additionally, these parameters characterize changes in earthquake rates (in time domain) and earthquake densities (in space domain), which together constitute an important component of a probabilistic seismic hazard assessment (PSHA).

The physics-based approach in turn requires information about other seismological attributes such as seismic phases, and also geophysical parameters such as the slip distribution over the ruptured areas, regional stresses, etc. The applied model in this dissertation computes the changes in Coulomb static stress ( $\triangle \mathrm{CFS}$ ) to analyse the interaction between earthquakes, or between earthquakes and the potential rupture planes in the Earth's crust. To analyse the aftershock triggering, I study static stress changes due to (1) earthquakes with a constrained slip distribution on a fault plane, and (2) slip processes over an extended area of a subduction interface. This 
approach involves modelling the mentioned slips and also improving the earthquake hypocentral location in order to obtain reliable results. The mentioned approaches, are implemented solely or together in the study of four earthquake sequences with different nature and in different locations of the world:

(I) A swarm sequence of low magnitude earthquakes in southern Spain is modelled using the statistical approach, and during different periods. I characterize this swarm with a predominant and continuous aseismic forcing starting at the pre-activity period, that it accelerates during the main-activity period. I propose the presence of an additional aseismic source in the area, that induce the swarm activity together with the tectonic loading and seismic triggering.

(II) A mainshock-aftershock sequence that starts with a large earthquake doublet in northwestern Iran is studied via both statistical and $\triangle \mathrm{CFS}$ modellings. We found less energetic events associated to the aseismic forcing. The coseismic $\triangle \mathrm{CFS}$ triggering of the doublet agrees with the hypocentral location of deeper aftershocks.

(III) The possibility that a shallow crustal mainshock-aftershock sequence in southern Mexico is triggered by the aseismic subduction processes (tectonic loading and slow-slip event) is studied through $\triangle \mathrm{CFS}$ modelling. The result shows that the triggering impact of the tectonic forcing before the slow-slip events is not evident but cannot be ruled out. On the contrary, the early phase of the slow-slip event presents a clear favouring influence, though small on triggering this sequence.

(IV) A thrust earthquake in southern Mexico is studied using both statistical and $\triangle \mathrm{CFS}$ modellings. The spatiotemporal variation of earthquake rate in the subduction interface, prior to and after this earthquake is modelled and incorporated into PSHA. A small rise in the annual exceedance rate of the strong ground motion highlights the importance of updating earthquake rates for seismic hazard assessments.

The studies that I carry out along this thesis, provide a more insightful description of some specific earthquake sequences and the aspects of their origin. However, the achieved conclusions are of use in the study of other earthquake sequences with similar seismotectonic conditions. It is also of great importance for the seismic hazard assessments, to include the parametrized spatiotemporal variation of the earthquake frequencies in the updating methods. 


\section{Resumen}

Los terremotos son fenómenos naturales que liberan una gran cantidad de energía, la cual viaja en forma de ondas sísmicas que se propagan en todas las direcciones desde el interior de la Tierra. Cuando estas ondas alcanzan la superficie terrestre, se genera una sacudida sísmica del suelo cuyas consecuencias son a menudo perjudiciales para el ser humano, el medio ambiente y el medio construido. Los geofísicos estudian las causas de los terremotos para conocer mejor cómo se producen y mitigar esos efectos adversos. Para ello, consideran que los terremotos no son eventos aislados, sino que se relacionan e interaccionan con una serie de terremotos con los que comparten la misma ventana espaciotemporal y presentan características comunes o similares. Esta tesis trata del estudio de series sísmicas. Considero dos metodologías diferentes: la sismología estadística y los métodos basados en procesos físicos.

El enfoque estadístico considera que un terremoto es un objeto puntual, con atributos que aparecen en los catálogos sísmicos, tales como las coordenadas hipocentrales, el tiempo origen y la magnitud. Estos atributos se tratan como variables aleatorias que siguen distribuciones de probabilidad. Por tanto, la calidad y homogeneidad del catálogo sísmico disponible afecta directamente a la validez e idoneidad de los modelos estadísticos que describen las distribuciones de probabilidad de los atributos de los terremotos. En esta tesis utilizo varios modelos estadísticos en ventanas espaciotemporales finitas y los aplico de forma individual o combinada, según cada caso estudiado. Por ejemplo, para estudiar la frecuencia de terremotos de una magnitud dada se usa la ley de Gutenberg-Richter y para analizar la variación temporal de ocurrencia de terremotos se aplica el modelo de Omori-Utsu y el modelo de secuencia de terremotos de tipo epidémico (en inglés epidemic type aftershock sequence, ETAS). El modelo ETAS es un proceso puntual en el cada sismo representa un punto con los atributos mencionados. Este modelo asume que todos los eventos pueden disparar réplicas potencialmente, como en un proceso de ramificación (en inglés, branching process). La aplicación del modelo ETAS proporciona estimaciones separadas de las tasas de actividad sísmica de fondo y de réplicas, que se asocian a diferentes tipos de fuentes, que son, respectivamente: (1) fuentes asísmicas como la carga tectónica, intrusiones rápidas de magma o fluidos in eventos de deslizamiento lento transitorios; y (2) fuentes sísmicas, como el disparo elástico entre dos terremotos, lo cual controla la ratio de ramificación. Las respectivas contribuciones de fuentes sísmicas y asísmicas, así como sus variaciones espaciotemporales, son determinantes para nuestro conocimiento de la naturaleza de series sísmicas. En suma, el resultado de la modelización estadística indica la naturaleza de los procesos de ramificación mediante parámetros cuantitativos. Además, estos parámetros caracterizan los cambios de tasas sísmicas (en el dominio temporal) y de densidad de terremotos (en el domino espacial), que son dos ingredientes importantes de la evaluación de la peligrosidad sísmica probabilista (en inglés probabilistic seismic hazard assessment, PSHA). 
El enfoque basado en procesos físicos, en cambio, requiere disponer de información sobre otros atributos sismológicos como fases sísmicas de sismogramas (para el proceso de relocalización previo), la distribución de deslizamientos sobre el plano de ruptura, el patrón regional de esfuerzos, etc. En esta tesis aplico el modelo de transferencia de esfuerzos estáticos de Coulomb (del inglés, Coulomb failure stress $\triangle \mathrm{CFS}$ ) para analizar la interacción entre terremotos, o entre un terremoto y posibles planos de ruptura en la corteza terrestre. Con objeto de analizar el disparo de réplicas (en inglés, aftershock triggering), estudio los canmbios de esfuerzos estáticos debidos a (1) eventos con una distribución de deslizamiento en el plano de ruptura determinada; y (2) procesos de deslizamiento sobre áreas extensas del interfaz de subducción (en inglés, subduction interface). Este enfoque supone modelizar los deslizamientos mencionados y mejorar la localización hipocentral para llegar a obtener resultados fiables. Los métodos empleados se aplican de manera individual o conjunta en el estudio de cuatro secuencias sísmicas, de distinta naturaleza que tienen lugar en diferentes zonas del mundo:

(I) una serie sísmica de tipo enjambre, formada por muchos sismos de baja magnitud, que ocurre en el sur de España. La serie se divide en tres partes y se estudia con métodos estadísticos. Caracterizo este enjambre principalmente como resultado de un agente generador asísmico continuado, que se acelera en la fase de mayor actividad de la serie. Propongo la presencia de una fuente adicional en la zona, además de la carga tectónica y de disparo entre terremotos, que induce la actividad de tipo enjambre.

(II) una secuencia de sismo principal y réplicas que se inicia con un doblete de terremotos grandes en el noroeste de Irán. En este caso se usa, de forma combinada, el método estadístico y la modelización de cambio de esfuerzos. Encuentro que los eventos menos energéticos están asociados a un agente generador asísmico. La secuencia de réplicas disparada por el doblete está de acuerdo con la localización hipocentral de las réplicas más profundas.

(III) una serie cortical de tipo sismo principal y réplicas que ocurre en el sur de México. En este caso se investiga la hipótesis de que la serie sea disparada por un proceso de subducción asísmico (carga tectónica y eventos de deslizamiento lentos, o en inglés slow-slip events, SSEs) se estudia mediante modelización de $\triangle \mathrm{CFS}$. Los resultados muestran que el impacto del disparo de la carga tectónica antes del evento de deslizamiento lento no es evidente, pero tampoco se puede descartar. Por contra, la fase inicial del evento de deslizamiento lento presenta una influencia favorable leve pero clara para el disparo de esta serie sísmica.

(IV) otra serie asociada a un terremoto grande en la zona de subducción de México. En este caso se modelizan los esfuerzos de Coulomb para determinar el posible disparo de las dos réplicas principales, que al parecer rompen diferentes asperezas cercanas a la ruptura principal. Seguidamente se aplica el método estadístico para determinar la variación espaciotemporal de tasas sísmicas en la zona la interfaz de la subducción antes y después de la ocurrencia de la serie sísmica. Los correspondientes mapas de tasas sísmicas se usan en sendos cálculo probabilistas de peligrosidad sísmica para calcular las tasas de excedencia de determinados niveles de movimiento fuerte, observándose un pequeño aumento de la aceleración esperada con las tasas actualizadas después de la serie. 
Los estudios desarrollados en esta tesis proporcionan una descripción más esclarecedora de determinados aspectos específicos de las series analizadas. Sin embargo, los procedimientos y conclusiones alcanzados se pueden utilizar en el estudio de otras series sísmicas en todo el mundo. Asimismo, se apunta el interés de incorporar las variaciones espaciotemporales de parámetros de sismicidad (tasas de actividad sísmica) para los métodos de estimación de peligrosidad que incorporan actualizaciones temporales de dichos parámetros. 



\section{Contents}

1 Introduction 1

1.1 Preface . . . . . . . . . . . . . . . . . . . . 1

1.2 Theoretical framework . . . . . . . . . . . . . . . 3

1.2.1 Types of earthquake sequences . . . . . . . . . . . . . . 4

Mainshock-aftershock .............. . 4

Swarm . . . . . . . . . . . . . . 5

1.2.2 Natural origin of earthquake sequences . . . . . . . . . . 5

1.2.3 Earthquake rate . . . . . . . . . . . . . . . 6

1.2.4 Exceedance rate of the ground motion . . . . . . . . . . . . 7

1.3 Research context . . . . . . . . . . . . . . . . . 11

1.3.1 Swarm-type sequence . . . . . . . . . . . . . . . . 11

The 2012-2013 Torreperogil-Sabiote sequence, southern Spain 11

1.3.2 Mainshock-aftershock sequence; crustal faulting . . . . . . . . . 12

The 2012 Ahar-Varzeghan sequence, north-western Iran . . . . 12

The 2001 Coyuca sequence, southern Guerrero, Mexico . . . . 13

1.3.3 Subduction sequence; large thrust earthquake . . . . . . . . . . . . . 14

The 2014 Papanoa sequence, southern Guerrero, Mexico . . . 14

2 Methodology 15

2.1 Statistical approach . . . . . . . . . . . . . . . . 15

2.1.1 Magnitude-frequency distribution . . . . . . . . . . . . . . . . . . 15

2.1.2 Seismicity rate modelling . . . . . . . . . . . . . . 21

Hazard function . . . . . . . . . . . . . . 22

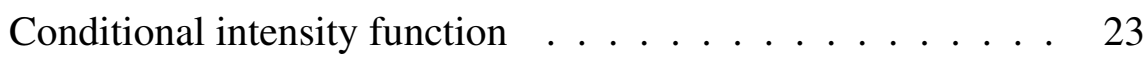

Stationary rate . . . . . . . . . . . . . . . 24

2.1.3 Omori-Utsu aftershock rate . . . . . . . . . . . . . . 26

2.1 .4 Temporal ETAS modelling . . . . . . . . . . . . . . . . 28

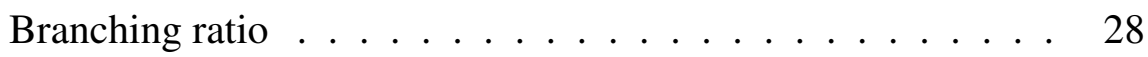

2.1.5 Seismicity density modelling . . . . . . . . . . . . . . 31

Kernel function . . . . . . . . . . . . . 31

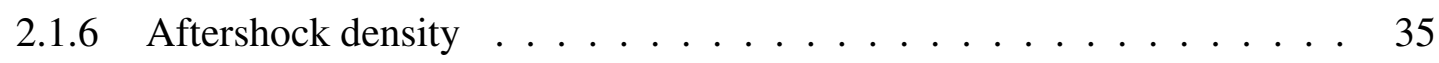

2.1.7 Spatiotemporal ETAS modelling . . . . . . . . . . . 36 
2.2 Earthquake re-location $\ldots \ldots \ldots \ldots \ldots \ldots$

2.3 Static stress modelling . . . . . . . . . . . . . . . . . . 39

2.3.1 Earthquake source modelling . . . . . . . . . . . . . . . . 42

2.3.2 Coulomb failure stress change . . . . . . . . . . . . . . . . . . 44

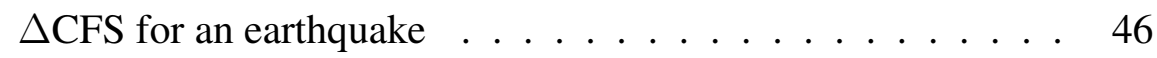

$\triangle \mathrm{CFS}$ for optimum ruptures $\ldots \ldots \ldots \ldots$

2.4 Probabilistic seismic hazard estimation . . . . . . . . . . . . . . . 51

2.4.1 Earthquake epicentre density rate estimation . . . . . . . . . . . . . . . 52

2.4.2 Exceedance rate of the strong ground motion . . . . . . . . . . 53

3 Swarm-Type Sequence $\quad 57$

"Statistical analysis of the 2012-2013 Torreperogil-Sabiote seismic series, Spain." ............................ 59

4 Mainshock-aftershock sequence; crustal faulting(I) 61

"Analysis of the 2012 Ahar-Varzeghan (Iran) seismic sequence: Insights from statistical and stress transfer modeling." . . . . . . . . . . . . 63

5 Mainshock-aftershock sequence; crustal faulting (II) 65

"The 2001 Coyuca seismic sequence." . . . . . . . . . . . . . . . . . . . . 67

5.1 Introduction . . . . . . . . . . . . . . . . . 67

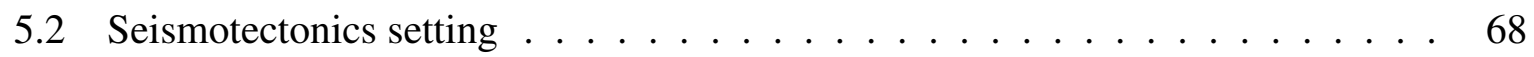

5.2 .1 Guerrero seismic gap . . . . . . . . . . . . . . . . . . . 68

5.2.2 Slow-slip events in Guerrero . . . . . . . . . . . . . . . . . . . . . . 69

5.2.3 2001-2002 slow-slip event . . . . . . . . . . . . . . . . 69

5.3 Data . . . . . . . . . . . . . . . . . . . . . 70

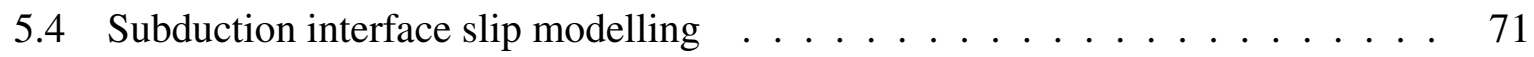

5.4.1 Phase-I: back-slip modelling . . . . . . . . . . . . . . . . . 71

5.4 .2 Phase-II: slow-slip modelling . . . . . . . . . . . . . . . . . 72

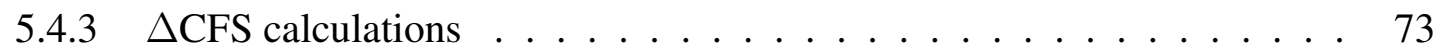

5.5 Discussion and conclusions . . . . . . . . . . . 76

6 Subduction sequence; large thrust earthquake $\quad 81$

"Analysis of the 2014 Mw 7.3 Papanoa (Mexico) earthquake: Implication for seismic hazard assessment." . . . . . . . . . . . . . . . . . 83

7 Summary and Conclusions $\quad 85$

$\begin{array}{ll}\text { References } & 93\end{array}$ 


\section{List of Figures}

1.1 Temporal (left) and geographical (right) distribution of magnitudes, related to the 2012 Ahar-Varzeghan earthquake sequence in north-western Iran. The red and orange crosses refer to the doublet. . . . . . . . . . . . . . . . . .

1.2 Temporal (left) and geographical (right) distribution of magnitudes, related to the earthquake sequence of 2014 Papanoa in Guerrero, Mexico. The red cross shows the mainshock.

1.3 Temporal (left) and geographical (right) distribution of magnitudes, related to the earthquake sequence of 2012-2013 Torreperogil-Sabiote in southern Spain. . . . . .

1.4 The surface projection of the density rate of subduction earthquakes for magnitude increments within $m \in[3.8,8.2]$, and between 1992 and 2018 in the researched zone of chapter 6

2.1 Up: the black line shows an example for the standard form of the Gutenberg-Richter law. The $a$-value and $b$-value are calculated using maximum likelihood estimation method (see Box 2.1) for the earthquakes in southern Spain (Figure 2.2) covering magnitudes within the catalog or [3.0,6.8]. The red line presents the low-limit truncated Gutenberg-Richter law, where a $m_{c}=4.0$ is applied to the same database. The blue triangles stands for the observed annual number of events $\dot{n}_{m}$ and the empty circles show the annual cumulative number of events $\dot{\mathcal{N}}(m)$. Down: the double-truncated form with two different maximum limits for comparison, where $m_{c}=4.0$ in both estimations $: m_{\max }=7.0$ (left) and $m_{\max }=6.5$ (right). . . . .

2.2 Earthquakes with magnitude $M_{\mathrm{w}} \geq 3.0$ in south-eastern of Spain (IGN \& UPM, 2013) between years 1048 and 2011 . . . . . . . . . . . . . . . . 20

2.3 The user interface of the designed application tool for spatial estimation of $b$-value. 20

2.4 The spatial distribution of Gutenberg-Richter parameters. . . . . . . . . . . . . . 21

2.5 The annual number of earthquakes in unit of area $\left(\mathrm{km}^{2}\right) \ldots \ldots \ldots 21$

2.6 Comparison between the shapes of two most commonly used Kernel functions; Gaussian function in dashed lines and IPL function in solid lines. Influence of the bandwidth $\mathrm{H}$ in smoothing of both functions (a), and the influence of the $q$-value in the IPL function $(b) \ldots \ldots \ldots \ldots \ldots$

2.7 Black dots show the mean values $\overline{\mathrm{DTN}}_{\text {min }}$, and are accompanied by blue lines that represent the estimated $\pm \sigma$ for the normal distribution associate to each magnitude class $m$. The red line is the best exponential fit for $\overline{\mathrm{DTN}}_{m i n}(m)$. This figure is estimated applying the earthquakes in the catalog of National Seismological Service of Mexico along the southern coast with epicentre between longitudes $96^{\circ} \mathrm{W}$ and $105^{\circ} W$ (see Figure 6 in chapter 6$) \ldots \ldots \ldots \ldots$

2.8 A comparison between the spatial probability density values in equation (2.51) for weighting parameters $0,0.5,1.0$ and 5.0 and different $\Theta$, with $\mathrm{H}=1$ and $q=1.5$. . 
2.9 Surface projection of the annual density of subduction interface earthquakes for magnitude increments $m=4.0$ with $\pm 2 \sigma_{m}$ between 1992 and 2018 in the research zone of chapter $6 . \ldots \ldots \ldots \ldots \ldots$

2.10 Graph of travel times of seismic phases identified in the IASPE 1991 Seismological Tables. This figure is fromStacey et al. (1977), pp 274, and the coloured features are added later. The red dashed curves highlight the $\mathrm{P}$ and $\mathrm{S}$ travel times. The two-sides arrow shows the difference between arrival time of seismic phases $\mathrm{P}$ and $\mathrm{S}$ for radial distance of 60 degrees. . . . . . . . . . . . . . . . . . .

2.11 The stress component $\sigma_{i j}$ showing the force acting in the direction of $i$ on an unit area of a plane with normal $j$ for positive side of volume increment (a). An unit of area with normal $\hat{\mathbf{n}}$ and its three orthogonal components of area (b). A force $\overrightarrow{\mathbf{T}}_{\hat{\mathbf{n}}}$ and its three orthogonal components acting on the unit area $\hat{\mathbf{n}}$ (c). The vectors $\sigma_{n}$ and $\sigma_{s}$ are the normal and the tangential components respectively. . . . . . . . . 40

2.12 Simple recurrence models by Shimazaki \& Nakata (1980). . . . . . . . . . . . . .

2.13 Schematic view of a simple a rectangular fault. The strike angle is the angle that the surface projection of the fault's upper edge makes with the north direction $\left(0^{\circ} \leq\right.$ strike angle $<360^{\circ}$ ) and in a way that the dip direction stays on its right hand side. The dip angle is measured from the surface. The rake angle is the slip angle on the fault plane, where $\lambda=0^{\circ}$ indicates a pure left-lateral slip, $\lambda=180^{\circ}$ a pure right-lateral slip, $0^{\circ}<\boldsymbol{\lambda}<180^{\circ}$ shows upward slip and $-180^{\circ}<\boldsymbol{\lambda}<0^{\circ}$ downward slip. 42

2.14 Examples of slip model solved for previous earthquakes worldwide, and downloaded from Finite-Fault Earthquake Source Model Database (Mai \& Thingbaijam, 2014).

2.15 The 2D ellipsoidal distribution (a) and the same distribution with cosine tapering (b). 43

2.16 Displacements because of shear strains . . . . . . . . . . . . . . . 44

2.17 Shear and normal stresses on a plane that has a positive angle of $\beta$ with the direction of $\hat{\mathbf{x}}_{1}$ for extension-positive (a), and compression-positive (b) coordinate systems. .

2.18 A cropped image form the latest production of WSM project which was published in 2016 (Heidbach et al., 2016, 2018). As it is shown in the legend, different type of data were used for extracting the information on the regional stress orientations.

2.19 The Mohr circle can be used for showing the amount of shear and normal stresses for different plane orientations. The positive rotation in the Mohr circle is always anticlockwise . . . . . . . . . . . . . . . .

2.20 The Mohr circle for the compression-positive system. For the extension-positive system the circles and the black line has to be mirrored with respect to the $\sigma_{s}$ axis. The positive angle in the Mohr circle is always anticlockwise and the sign of $r$ is respected (presented in bold arrow) for finding the normal and shear stresses (dotted arrows and dashed arrows respectively). . . . . . . . . . . . .

2.21 The black stars presents an example of six events with magnitude $m_{j}$, where the epicentre error and other eight probable location of event $E_{j 2}$ are shown in blue stars. The red dot presents an example of 25 grid points $G_{k}$ around calculation site $\mathcal{S}$ shown as a green triangle. The coordinate $(x, y)$ is equation (2.79) is set for $G 12$ as an example. The increment area related to grid point $G 9$ is shown as an instance. 
2.22 The attenuation of peak ground acceleration (PGA) by distance and for magnitudes between $M_{\mathrm{w}} 5.0$ and $M_{\mathrm{w}} 8.0$ using the ground motion prediction equation proposed by Arroyo et al. (2010) for Mexican interplate earthquakes. . . . . . . . . . . . . .

2.23 Depth distribution versus magnitude for shallow earthquakes (depth $\leq 45 \mathrm{~km}$ ) with magnitudes between $M_{\mathrm{w}} 2.9$ and $M_{\mathrm{w}} 5.5$ and within latitudes $\left[15^{\circ}, 20^{\circ}\right]$ and longitudes $\left[96^{\circ}, 105^{\circ}\right]$ in Mexican catalog. The black stars show the average depth versus magnitude. . . . . . . . . . . . . . . . . .

5.1 Tectonic setting and Cocos plate subducting velocities are shown together with the epicentre and estimated ruptured areas by large megathrust earthquakes near the Guerrero segment in the Mexican subduction. The left caption is adopted from UNAM Seismology Group (2015) and the right caption is from Radiguet et al. (2012). 68

5.2 Relocated events between $8^{\text {th }}$ October and $31^{\text {st }}$ December 2001. Focal mechanism are from Table 5.1.

5.3 Inter-SSE coupling index by Radiguet et al. (2016). The blue circle and the yellow triangle indicate the epicentre of the Coyuca mainshock given by SSN and CMT, respectively. Dashed blue line represents a 200-km long cross section perpendicular to the MAT and passing by these epicentral locations. Think black contours represent the depth to the interface surface. . . . . . . . . . . . . . . .

5.4 Back-slip $\left(\right.$ rake $\left.=-90^{\circ}\right)$ amount during 38 months and applying a constant shortening of $0.5 \mathrm{~cm} \cdot \mathrm{mo}^{-1}$ between longitudes $-98.5^{\circ}$ and $-102.5^{\circ}$. Back-slip $=38 \times 0.5 \times$ coupling index (in Figure 5.3) in cm. . . . . . . . . . . . . . . . .

5.5 A simplified model of the total slip distribution in 2001-2002 SSE, where each zone, represented by different colour, is assigned by its minimum slip value in the original model by Radiguet et al. (2012). The red dashed line shows a total slip of $\sim 0 \mathrm{~cm}$ along the coast as in the original model. Rest of the symbols are as in Figure 5.3. .

.6 The $\triangle$ CFS for the southward nodal plane by SSN, on the cross section in Figure 5.3 and applying model-I (up), and 10\% of total slip in model-II (down). Dashed black line indicates $\triangle \mathrm{CFS}=0$.

5.7 The $\triangle \mathrm{CFS}$ for the southward nodal plane by SSN, on the cross section in Figure 5.3 (here is zoomed) and applying the summation of slip model in model-I and 10\% of total slip in model-II. Yellow dots show the relocated events in Figure 5.2. Dashed black line indicates $\triangle \mathrm{CFS}=0 \ldots \ldots \ldots \ldots \ldots$

5.8 The two clusters after re-location of aftershocks. Cluster-I has 99 earthquakes (a) and cluster-II has 103 earthquakes (b). Both clusters have a southwestward dipping. Blue and green lines represent 100-km long cross sections perpendicular and parallel to the MAT, respectively. . . . . . . . . . . . . . . . . . .

5.9 Distribution of $\triangle$ CFS for model-II with $10 \%$ of slow-slip in 2001-2002 with rake of $95^{\circ}$, and for focal mechanism $101^{\circ} / 33^{\circ} / 90^{\circ}$ (SSN), on the two cross sections in Figure 5.8. The hypocentral location of cluster-I (left) and cluster-II (right) are projected on both profiles.

5.10 The $\triangle$ CFS for southward nodal plane by SSN, on the cross section in Figure 5.3 (here is zoomed) and applying the summation of slip model in model-I and $40 \%$ of total slip in model-II. Rest of the symbols are as in Figure 5.7. . . . . . . . . . 
5.11 A zoomed caption of Figure 5.3 (a), and Figure 5.5 (b), for a better resolution in showing the west-side cross sections (profiles A, B, C and D) and the east-side cross sections (profiles I, H, G and F), with horizontal distances of 20, 15, 10 and $5 \mathrm{~km}$, respectively, to the main cross section (the same as in Figures 5.3 and 5.5) or profile E. Blue dots indicate the upper centre of each profile. . . . . . . . . . .

5.12 The $\triangle$ CFS for southward nodal plane by SSN, on the cross sections in Figure 5.11 and within the zoomed window as in Figure 5.10. The applied slip models is the summation of slip in model-I and $40 \%$ of total slip in model-II. Dashed black line indicates $\triangle \mathrm{CFS}=0 \ldots \ldots \ldots \ldots \ldots \ldots \ldots$

7.1 Results of the ETAS modelling in the time domain for the earthquake sequences of 2012-2013 Torreperogil-Sabiote: main activity phase (a), 2012 Ahar-Varzeghan (b), and 2014 Papanoa $(\mathrm{c}$ and $\mathrm{d}) . \ldots \ldots \ldots$. . . . . . . . . . . . . . 86

\section{List of Tables}

5.1 Hypocentre location and focal mechanism solutions for $M_{\mathrm{w}} 5.8$ Coyuca mainshock on $8^{\text {th }}$ October 2001 by Servicio Sismólgico Nacional (SSN) of Mexico and Global Centroid Moment Tensor (CMT) catalog. . . . . . . . . . . . . . . . . . . . . . 67 


\section{Symbols}

I hereby introduce a list of used parameters in the body of this dissertation. However, chapters 3, 4 and 6 include published articles and some parameters and formulas in these chapters may come with different notations and not as below.

- $\alpha \quad$ a productivity parameter

- $\beta \quad \beta$-value in the Gutenberg-Richter law

- $\gamma \quad$ a Kernel bandwith parameter

- $\Theta \quad$ azimuth along which the asymmetric Kernel function is aligned

- $\Lambda$ the exceedance rate of the ground motion

- $\lambda \quad$ earthquake rate

- $\lambda \quad$ Lamé parameter

- $\lambda \quad$ rake angle

- $\mu \quad$ background rate

- $\mu_{f} \quad$ static stress coefficient

- $\nu \quad$ modelled number of aftershocks / Poisson's ratio

- $\rho \quad$ earthquake density (in unit of area)

- $\sigma \quad$ standard deviation / stress coefficient

- $\tau \quad$ time lag / shear stress

- $\Phi \quad 2$ dimensional zone of study / seismic phase

- $\Psi \quad$ a known ground motion value

- $\psi \quad$ the ground motion level 
- $\omega$ probability of belonging to the background population

- $\mathcal{A} \quad$ ruptured area by an earthquake

- $A_{\Omega} \quad$ normalization constant in temporal ETAS

- $A_{\Phi} \quad$ normalization constant in spatiotemporal ETAS

- $a \quad$ a-value in the Gutenberg-Richter law

- $b \quad$ b-value in the Gutenberg-Richter law

- $c \quad$ an Omori-Utsu parameter

- $d \quad$ a Kernel bandwith parameter

- $d A \quad$ area increment

- $d V \quad$ volume increment

- E mathematical expectation

- $e \quad$ strain coefficient

- $\mathcal{F} \quad$ earthquake source

- F cumulative distribution function or $\mathbf{c d f}$

- $f \quad$ probability density function or pdf

- $\mathcal{G} \quad$ pdf for spatial distribution of aftershocks related to a mainshock

- $\mathrm{G} \quad$ rigidity modulus

- $\mathrm{H}$ the bandwidth of the Kernel function

- $\mathcal{H}_{t} \quad$ seismicity history up to time $t$

- h hazard function

- $\mathcal{K} \quad$ productivity

- K Kernel function

- $\mathcal{L} \quad$ length of a rupture plane

- $L \quad$ likelihood function

- $L L \quad$ logarithm of the likelihood function

- $\mathcal{M}_{0} \quad$ seismic moment of an earthquake

- $\mathbb{M} \quad$ set of magnitude classes

- m mainshock magnitude 
- $m_{\max } \quad$ maximum magnitude

- $m_{c} \quad$ completeness magnitude

- $N \quad$ total number of events

- $\mathcal{N}(m) \quad$ cumulative number of events with magnitudes $\geq m$

- $\mathbb{N} \quad$ set of frequency of each magnitude class in $\mathbb{M}$

- $n_{\mathcal{F}} \quad$ number of earthquake sources in area-source PSHA

- $n_{e} \quad$ half-size of the smoothing window

- $P$ probability function

- $p \quad$ an Omori-Utsu parameter

- $\mathrm{p} \quad$ pressure within matter

- $q \quad$ a Kernel parameter

- $S \quad$ survival function

- $\mathrm{S}_{0} \quad$ cohesion or internal strength of the rock

- $\mathcal{S} \quad$ calculation site

- s $\quad$ slip size

- $\mathcal{T} \quad$ pdf for distribution of aftershock time-lag related to a mainshock

- $T_{i n i} \quad$ starting point in time

- $T_{\text {end }} \quad$ ending point in time

- $t_{m} \quad$ time period of recording events with magnitude $m$

- $\mathrm{U}$ displacement field within the rock

- $\mathcal{W} \quad$ width of a rupture plane

- $\mathrm{w}$ directivity weight in an asymmetric Kernel function

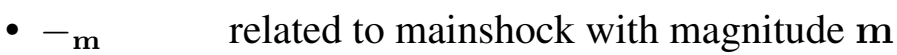

$\begin{array}{ll}-{ }_{\mathbf{a}} & \text { related to aftershocks }\end{array}$

- $-{ }_{\mathbf{a}, \mathbf{m}}$ related to aftershocks of a parent with magnitude $\mathbf{m}$ 



\section{Chapter 1}

\section{Introduction}

\subsection{Preface}

An earthquake is a natural event associated with the rupture of rocks that compose the relatively brittle parts of the Earth's crust and lithospheric mantle. This rupture usually occurs suddenly and involves the release of a considerable amount of energy during seconds to several minutes that travels in all directions in the form of seismic waves. When these waves reach the surface of the Earth, they induce ground shaking and eventually ground failure (liquefaction, landslides, cracks) that can cause adverse effects on human lives and activities.

The physical consequences of earthquakes are damage to the built and natural environment that can cause death and injuries to human beings, as well as economic losses. The social and economic cost of such hazardous events depend on many factors. A detailed knowledge about these factors can be used to reduce the level of negative impact. Geophysical factors that determine the earthquake origin time, occurrence location and size cannot be controlled, albeit they can be surveyed, investigated, estimated and monitored.

Indeed, most of the times, an earthquake is not an independent event, but is part of a sequence consisting of several earthquakes. This fact is empirically acknowledged and well-understood by people experiencing an earthquake, who still run outdoor and remain there for a few nights after a significantly perceived earthquake, as they are concerned whether the next event is going to strike their lives or not. Thus, it is important to study causes and effects of earthquakes within the context of earthquake sequences.

For seismologists, identifying an earthquake sequence is usually intuitive and requires experience. Sometimes, they start by a slight rise in seismic activity (foreshock activity) and then a dominant earthquake (in terms of size) or mainshock occurs (e.g., 1975 Haicheng earthquake with $M_{\mathrm{w}} 7.3$ in China, 2015 Illapel earthquake with $M_{\mathrm{w}} 8.3$ in Chile, 2019 Ridgecrest earthquakes with $M_{\mathrm{w}} 7.1$ in California) and sometimes the earthquake sequence starts with a large event without any foreshock activity (e.g., 2004 Parkfield earthquake with $M_{\mathrm{w}} 6.0$ in California). What commonly comes next is a population of earthquakes (aftershocks) with close spatiotemporal distances to 
the mainshock, while they usually become smaller and less frequent in time. There also exist earthquake sequences with no dominant shock (swarms) and yet they can cause considerable social alarms, as the 2012-2013 Torreperogil-Sabiote in Jaén and the 2013 Castor in the Gulf of Valencia, Spain (e.g., Yazdi et al., 2017; Ruiz-Barajas et al., 2017).

Since the past two decades, many investigations focus on the characterization of different earthquake sequences, studying the interaction between earthquakes, their triggering effects and their applications in earthquake forecast and prediction. Some methods are based on statistical clustering theories to forecast the occurrence of earthquake activity rate within a future time span (e.g., Utsu \& Ogata, 1995; Zhuang et al., 2011). They include models in which all earthquakes have a single "parent" that triggers the whole sequence (e.g., Kagan, 1991; Felzer et al., 2004), and "cascade models", in which any earthquake can trigger its own "children" and the sequence display a cascade of earthquakes via a branching process (e.g., Ogata, 1998; Helmstetter \& Sornette, 2002). Statistical models help characterizing earthquakes clustering in time and space, but do not explore the underlying physics. Other methods consider the perspective of mechanical physics to address rupture dynamics, stress interactions between different earthquakes, coseismic stress changes, etc. (e.g., Harris, 1998; Stein, 1999; King \& Cocco, 2001). They narrow down the domain of earthquake forecast by providing more detailed physics-based explanation to distinguish triggered events from triggering events in a spatiotemporal frame (Scholz, 2019). Nevertheless, earthquake prediction, conceived as the exact estimate of occurrence time, size and location of an earthquake, is not yet possible. In practice, alternative approaches to earthquake forecast include the estimation of activity rates for different spatio-temporal frames (depending on the final application).

The activity rates are used in seismic hazard analyses, which give the exceedance probability of different strong ground motion levels expected in a given exposure time. Hazard-consistent ground motions are used for earthquake-resistant design (and other seismic risk applications). Target ground motion exceedance probabilities are defined based on construction life-time, building importance and occupancy. For instance, structures such as residential buildings are usually constructed to withstand a strong ground motions with $10 \%$ probability of exceedance in 50 years. In turn, the suggested exceedance probability to consider for special-importance structures such as hospitals is $5 \%$ in 50 years.

In earthquake forecast studies, the activity rates are typically estimated in the short-term, which generally requires characterizing the aftershock activity. The implementation of aftershock rates in seismic hazard assessments could benefit from the incorporation of more physics-based approach (impact of stress changes) and would involve a time-dependent evaluation of strong ground motion.

Although studying earthquake sequences is important as a strong socio-economical urge, it is also of a great value as it deepens our knowledge about physics of the Earth. Different research results suggest that earthquake generation and clustering present similar spatiotemporal patterns under similar geophysical circumstances. During decades, these similarities are shaped into empirical laws and models. Applying the existing models, either statistical or physics-based, 
reveals insightful information for uncovering the nature of earthquakes, especially in the lessexplored areas. This dissertation aims to contribute in this regard. Four earthquake sequences in different geophysical settings are addressed. These earthquake sequences are introduced in section 1.3, after a description of the theoretical framework.

\subsection{Theoretical framework}

As it was mentioned in section 1.1, the focus of this dissertation is the analysis of earthquake sequences. An earthquake sequence or earthquake series is a group of earthquakes that occur in a determined and limited space and time window. Earthquakes within an earthquake series supposedly share the same forcing source/s and their distributions in space and time exhibit a sequential pattern. In some occasions an earthquake sequence starts with one or more foreshocks that by definition are those earthquakes that occur prior to the largest earthquake of the sequence (i.e., the mainshock). Following a mainshock there are always smaller earthquakes or aftershocks that may continue for weeks, months or years, depending on the magnitude of the mainshock and the geologic circumstances. However, it is important to remember that the definitions of these terms are not as unique and may vary depending on our understanding of the earthquake sequences generation and cause and effect relationship between earthquakes. For instance, the definitions of mainshock and aftershocks become more objective in statistical clustering theories that consider multiple cascade models in which an earthquake can be both mainshock and aftershock .

To present the theoretical framework of this dissertation, firstly, the two types of earthquake sequences (mainshock-aftershock and swarm) are defined in section 1.2.1. The importance of earthquake size as the key factor for categorizing the earthquake sequences is remarked. Secondly, the geo-tectonic settings that support the occurrence of each type of earthquake series are introduced briefly in section 1.2.2. The two types of earthquake sources (aseismic and seismic) are described. Therefore, considering the type of an earthquake sequence and available knowledge about its geological and geophysical conditions, we can make some preliminary suppositions about its possible causes. Such overview, guides us towards asking relevant questions and choosing appropriate methods for characterizing the earthquake activity in time and space domains.

In section 1.2.3, the importance of earthquake rate modelling and the classification of the earthquake population into background and aftershock rates are described. It is explained that how this classification matters at the time of earthquake rate analysis and the interpretations about earthquake generation processes. Indeed, analysing the spatiotemporal changes in earthquake rates is one of the principal focus in this dissertation. Finally, in section 1.2.4, the concept of seismic hazard is introduced, and the mathematical formulations for implementing earthquake rates in probabilistic seismic hazard within the scope of this dissertation are described. 


\subsubsection{Types of earthquake sequences}

\section{Mainshock-aftershocks}

An earthquake sequence is usually considered as a mainshock-aftershock sequence if there exists at least one earthquake with a dominant magnitude among a group of earthquakes in a defined spatiotemporal window. The event with a distinguishably largest magnitude is called the mainshock and the events the come afterwards and have smaller magnitudes are generally called aftershocks. It is supposed that the amount of subsequent aftershocks depends on the mainshock magnitude (Utsu, 1971). A mainshock with larger magnitude usually have much more number of aftershocks that continue for longer period of time, up to month or years. Omori (1894) proposes that the rate of aftershocks activity in a mainshock-aftershock sequence decays by an inverse power law during time.

The Båth law suggests that, in average, the second largest earthquake following the mainshock has a mean magnitude about 1.2 smaller than the mainshock magnitude (Båth, 1965). Thus, if the distribution of magnitude versus time is available, the mainshock should be easily recognizable. The mainshock-aftershock sequence shown in Figure 1.1 is studied further in chapter 4. This earthquake sequence in north-western Iran, starts with two mainshocks or a "doublet" with magnitudes of order 6.4 and 6.2, which are easily distinguishable from the following events in terms of size. The third largest earthquake right after the doublet has a magnitude of 5.6.
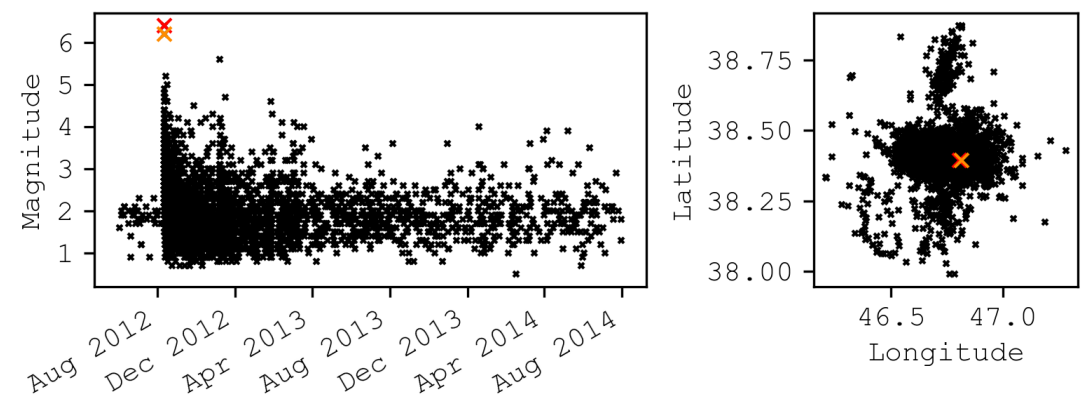

Figure 1.1: Temporal (left) and geographical (right) distribution of magnitudes, related to the 2012 Ahar-Varzeghan earthquake sequence in north-western Iran. The red and orange crosses refer to the doublet.
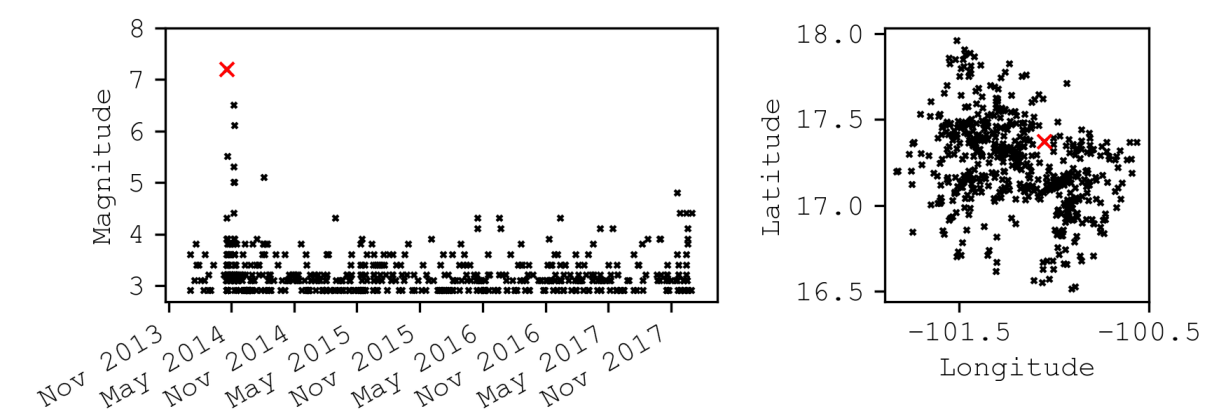

Figure 1.2: Temporal (left) and geographical (right) distribution of magnitudes, related to the earthquake sequence of 2014 Papanoa in Guerrero, Mexico. The red cross shows the mainshock. 
The mainshock-aftershock sequence shown in Figure 1.2 is studied in chapter 6. This earthquake sequence is related to subduction interface earthquakes in southern Guerrero (Mexico), following a large subduction thrust earthquake with a magnitudes of 7.3. The second largest earthquake in this series has a magnitude of 6.4 .

\section{Swarm}

On the contrary, with respect to a mainshock-aftershock type, in a swarm sequence, all the earthquakes have similar sizes with no remarkable magnitude which is greater than the others. Thus, tracing any mainshock and its subsequent aftershocks is not straightforward. Figure 1.3 shows a swarm sequence in southern Spain that is studied in chapter 3. This sequence started on late October 2012 and lasted for about 8 months. The largest event in 5 February 2013, has a magnitude of 3.9 which does not present any a considerable difference in comparison with the next largest magnitudes within the sequence.
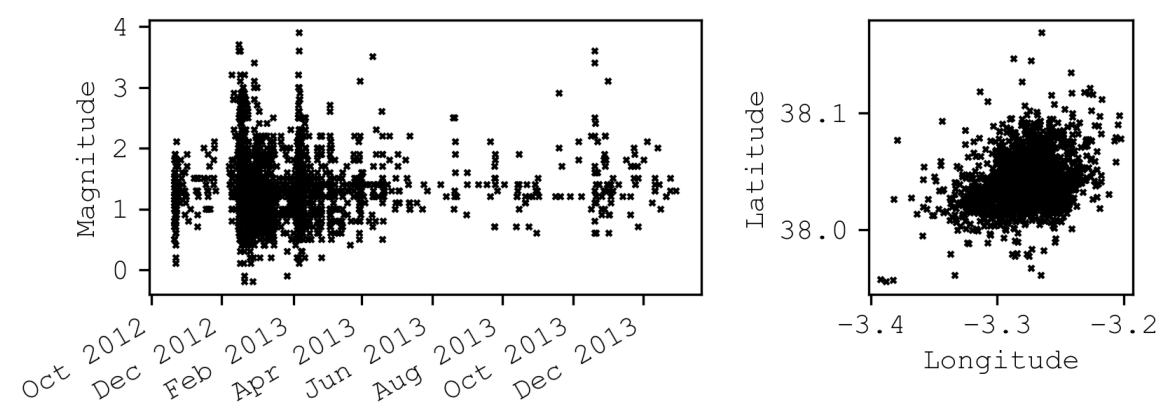

Figure 1.3: Temporal (left) and geographical (right) distribution of magnitudes, related to the earthquake sequence of 2012-2013 Torreperogil-Sabiote in southern Spain.

\subsubsection{Natural origin of earthquake sequences}

The lithosphere, which contains the Earth's crust, is divided in tectonic plates that are underlain by a semi-fluid asthenosphere. The viscous flow transmitted by the deep mantle convection currents to the upper mantle forces the tectonic plates continuously and yields a huge stress storage in the crust over time (Morgan, 1971; Stacey et al., 1977). The elastic rebound (Reid, 1910) of the accumulated stress in the crust, might produces sudden slips along the tectonic plate boundaries and pre-existing fractured areas in the solid lithosphere (i.e. faults). This process can also result in the generation of new fractures with simple or complex geometries within a fault system (Molnar et al., 2007). The fault ruptures that are directly related with the tectonic plate motions and the associated regional stress regime yield tectonic earthquakes. The occurrence of earthquakes, despite the causing source, affects the stress settings close to their ruptured areas. Such perturbations in the stress arrangement are able to induce further slip instabilities within the fault system (King et al., 1994). The amount of corresponding seismic stress changes is proportional to the earthquake size. These generated earthquakes are triggered by a seismic source, whereas the tectonic earthquakes have an aseismic source that is the tectonic loading. Earthquake sequences that begins with a relatively large tectonic earthquake/s, generally produce a vast amount of seismically triggered events. Such earthquake sequences are usually categorized as mainshock-aftershock type. 
Sometimes, the mechanical state of the seismogenic crust reaches to a new arrangement not because of the regional stress due to the tectonic forcing, nor the seismic stress due to previous earthquakes, but because of an increase in the pore fluid pressure $p_{\text {pore }}$ of the rocks. An intrusion of fluids into the rock pores induces higher pore pressure and drops the effective normal stress to the fault. These stress changes facilitate the fault rupture (seismic failure) (Nur \& Booker, 1972; Yamashita, 1999). Hence, pore pressure perturbations constitute another aseismic source of earthquakes that can be driven from an anthropogenic activity or not. This process usually provokes the occurrence of swarm series, strongly clustered, with no dominant magnitude and possible hypocenter migration due to the fluid movement (e.g., Hainzl \& Fischer, 2002; Vidale $\&$ Shearer, 2006). Likewise, magma rise and motion and its interaction with the surrounding rocks induces volcano earthquakes (Chouet, 1996) that also generate swarm-type activity (Hill, 1977). In this case, the magnitude difference between the largest and the second largest events is usually less than 0.5 magnitude units (McNutt, 2005).

Slow-slip earthquakes and fault creeping are other source of aseismic transient that can induce earthquake swarm activity with hypocentre migration (e.g., Lohman \& McGuire, 2007; Fasola et al., 2019). The slow-slip phenomenon includes processes that move smoothly along the fault surface with no seismic radiation or with very low frequency radiations (in comparison to the frequencies that a regular earthquake produces) Scholz (2019). Instead, fault creeping is a near-surface phenomenon, where the shear on a shallow fault is not accompanied by an earthquake. The creeping usually occurs in complex fractures and can be a response to large earthquakes on other faults (Harris, 2017).

\subsubsection{Earthquake rate}

In this dissertation, both statistical and physics-based methods are used for characterizing earthquake sequences and to understand their origin and nature, describing the distribution of earthquakes in time, space and magnitude domains. The spatiotemporal variation of the earthquake rate, here denoted by $\lambda$, is of high importance in this regard, because changes in the earthquake rate can be interpreted as changes in the causative geophysical processes. On the other hand, the estimation of earthquake rate is an essential part of in seismic hazard assessments. To study earthquake rates it is convenient to assume that an earthquake population comprises two contributions: background and aftershocks events. The term background earthquake refers to those events that are generated by aseismic sources such as tectonic loading forces, fluid or magma intrusions into the rock pores and stress transients such as slow slip or fault creeping activities. This population comprises the first generation of parents which can trigger aftershocks. The term aftershocks points to those earthquakes that are triggered seismically by the background earthquakes or even by previous aftershocks, and display a cascade process. Indeed, aftershocks (specially those with higher magnitudes) become the next generation of parents and can trigger secondary aftershocks. However, it is quite complicated to separate aftershocks and background earthquakes to determine if an earthquake is a parent, a child or both. And not only because each earthquake with any size can induce its own offspring, but also because the loading force causing a rupture can have a mixture of both aseismic and seismic origin. 
Statistical methods help modelling earthquake rates and estimating the overall contribution of background and aftershock populations. In unit of time we have:

$$
\lambda=\mu+\nu
$$

where, $\mu$ is the background rate and $\nu$ is the aftershock rate. Determining $\mu$ and $\nu$, which represent earthquakes with distinct nature, and analysing their variation in time and space can reveal considerable information about the changes in the ongoing geophysical processes. The earthquake rate variations can also be implemented in seismic hazard assessments. In the following section, the determinant role of earthquake rate as a component of probabilistic seismic hazard is explained.

On the other hand, with the scope of physics-based approach, the process that triggers an instantaneous or delayed aftershock is not the same. Moreover, the seismic triggering close to the mainshock rupture is different from the off-fault aftershocks at greater distances (Scholz, 2019). Some physics-based approaches model the aftershock rate variation in time and space domains due to coseismic stress changes (Dieterich \& Conrad, 1984). Indeed, physics-based modelling tries to uncover more detailed physical properties of an earthquake sequence and justify the spatiotemporal variation of activity rates.

\subsubsection{Exceedance rate of the ground motion}

Seismic hazard at a particular site $\mathcal{S}$ on the Earth's surface, refers to the exceedance probability of a ground motion intensity $\psi$ from a certain level $\psi^{*}$ due to the earthquake activity in the site's neighbourhood and in an exposure time. Thus, the estimation of seismic hazard involves the quantitative evaluation of the expected ground motion intensity at the site.

Regardless of the applied method to compute seismic hazard, (I) the earthquake source parameters, (II) the attenuation of seismic waves energy along their travelling path to the site and (III) the local soil amplification at the site contribute to the estimation of an expected ground motion value of $\Psi$.

The mathematical representation of the attenuation model is the ground motion prediction equation (GMPE), which estimates the level of ground motion using independent variables as the magnitude $M$, the earthquake-to-site distance $R$ and other quantitative and/or qualitative factors $Q$ (e.g., the style of faulting and soil type). The ground motion value $\Psi$, corresponding to a certain set of $M-R-Q$ values, is the basic outcome of a deterministic seismic hazard assessment (DSHA), which counts as a determinative component of seismic risk evaluations in urban areas (e.g., Yazdi et al., 2018b). This estimate has no further probabilistic considerations, apart from the uncertainty related to the GMPE.

However, in probabilistic seismic hazard assessment or PSHA, no determined scenario is minded. Instead, the exceedance probability of $\psi^{*}$ because of all potential earthquake sources, that can induce significant strong ground motion levels at the site $\mathcal{S}$, are considered. The exceedance probability of $\psi^{*}$ for one single earthquake (with magnitude $M$ at distance $R$ to the site) that 
causes an average strong ground motion level of $\Psi$ at the site is given by:

$$
P\left(\psi>\psi^{*} \mid M, R\right)=P\left(\Psi>\psi^{*}\right)=1-F_{\Psi, \sigma_{\psi}}\left(\psi^{*}\right),
$$

where $F_{\Psi, \sigma_{\psi}}(\psi)$ is the normal cumulative distribution function with mean value of $\Psi$ and standard deviation of $\sigma_{\psi}$, and its associated normal probability density function is:

$$
f_{\Psi, \sigma_{\psi}}(\psi)=\frac{1}{\sqrt{2 \pi \sigma_{\psi}^{2}}} \exp \left(-\frac{(\psi-\Psi)^{2}}{2 \sigma_{\psi}^{2}}\right) .
$$

Therefore the exceedance probability of $\psi^{*}$ can be written as:

$$
P\left(\psi>\psi^{*}\right)=P\left(\psi>\psi^{*} \mid M, R\right) P(M, R)=P\left(\Psi>\psi^{*}\right) P(M, R),
$$

where $P(M, R)$ is the occurrence probability for an earthquake with magnitude $M-\frac{\delta m}{2}<M \leq$ $M+\frac{\delta m}{2}$ at distance $R-\frac{\delta r}{2}<R \leq R+\frac{\delta r}{2}$ to the site, where $\delta m$ and $\delta r$ are the increments that are applied to discretize magnitude and distance, respectively. Let's assume that an earthquake source $\mathcal{F}$ is capable to generate $n m$ magnitude classes $m_{j}$ (earthquakes with magnitudes $m_{j} \pm \frac{\delta m}{2}$, where $j=1,2,3, \ldots n m$ ) and these earthquakes occur on $n r$ different distance increments $r_{k}$ (counts for earthquake-to-site distances of $r_{k} \pm \frac{\delta r}{2}$, where $k=1,2,3, \ldots n r$ ). Then, the exceedance probability of $\psi^{*}$ at site $\mathcal{S}$ due to an earthquake generated in source $\mathcal{F}$ is:

$$
P\left(\psi>\psi^{*}\right)=\sum_{j=1}^{n m} \sum_{k=1}^{n r} P\left(\Psi_{j k}>\psi^{*}\right) P\left(m_{j}, r_{k}\right),
$$

where, $P\left(\Psi_{j k}>\psi^{*}\right)=P\left(\psi>\psi^{*} \mid m_{j}, r_{k}\right)$ is the strong ground motion value because of magnitude class $m_{j}$ at distance increment $r_{k}$ and $P\left(m_{j}, r_{k}\right)$ is the associated occurrence probability for an earthquake with magnitude of $m_{j} \pm \frac{\delta m}{2}$ at distance $r_{k} \pm \frac{\delta r}{2}$ to the site. Based on the union rule of probability we have $P\left(m_{j}, r_{k}\right)=P\left(m_{j} \mid r_{k}\right) P\left(r_{k}\right)$ or $P\left(m_{j}, r_{k}\right)=P\left(r_{k} \mid m_{j}\right) P\left(m_{j}\right)$.

Nevertheless, in PSHA the objective is not to estimate the instant exceedance probability of $\psi^{*}$, because no one can predict the exact time that $\mathcal{F}$ produces an earthquake. Instead, in PSHA we deal with temporal uncertainties. Every source $\mathcal{F}$ at a certain time $t$ is associated with an earthquake rate $\lambda$, that represents an average for the total number of earthquakes in unit of time (usually a year). Using a very simplified condition, this average value can be estimated by $N / T$, where $N$ is the total number of earthquakes with due to source $\mathcal{F}$, during a long period of time $T$ (decades or a more). For a regular PSHA study, the total number of earthquake $N$, is taken from a catalog that (1) is homogeneous in terms of magnitude scale, (2) includes fully record of all applying magnitudes during $T$, and (3) is declustered and does not include any foreshock or aftershock. Notwithstanding, the applying approach to prepare such a catalog, and to estimate $\lambda$, the association of $\mathcal{F}$ to an earthquake rate, suggests that PSHA can be represented in terms of exceedance rate $\Lambda\left(\psi>\psi^{*}\right)^{1}$ with the same time unit definition as $\lambda$. Therefore if we estimate $\dot{n}\left(m_{j}, r_{k}\right)$, which is the number of earthquakes with magnitude $m_{j}$ at distance $r_{k}$ to site $\mathcal{S}$, in unit of time, we can estimate the exceedance rate:

$$
\Lambda\left(\psi>\psi^{*}\right)=\sum_{j=1}^{n m} \sum_{k=1}^{n r} P\left(\Psi_{j k}>\psi^{*}\right) \dot{n}\left(m_{j}, r_{k}\right) .
$$

\footnotetext{
${ }^{1}$ Using capital lambda $\Lambda$, is to avoid its confusion with the earthquake rate, denoted by $\lambda$.
} 
The term $\dot{n}\left(m_{j}, r_{k}\right)$ at any certain time $t$ is equal to $\lambda P\left(m_{j}, r_{k}\right)$ and we can write:

$$
\Lambda\left(\psi>\psi^{*}\right)=\lambda \sum_{j=1}^{n m} \sum_{k=1}^{n r} P\left(\Psi_{j k}>\psi^{*}\right) P\left(m_{j}, r_{k}\right),
$$

where the resulting $\Lambda\left(\psi>\psi^{*}\right)$ relies on the estimation of earthquake rate $\lambda$, and thus its associated spatiotemporal window. The relation between the exceedance rate and the exceedance probability of the ground motion $\psi^{*}$ is described in section 2.4. Hereby, it is explained how the estimation of exceedance rates $\lambda$ is carried out through two main approaches: considering area-sources or zones (areas with uniform seismic characteristics) or the zone-less approach. The fundamental difference is in the applied methods for estimation of $P(m, r)$ over the seismic area.

The area-source approach divides the seismic area into $n_{\mathcal{F}}$ individual sources that are typically 2D area-sources (sometimes they might be 1D linear-sources). Traditionally, each source $\mathcal{F}_{i}$, is defined with a uniform spatial distribution of earthquake rate and magnitude probability (Cornell, 1968). Defining such a homogeneous seismic zones implies:

(I) the probability density function for the magnitude-frequency $f_{i}\left(m_{j}\right)$ does not vary over the area-source which means that the magnitude probability does not depend on the distance $r_{k}$, thus $P_{i}\left(m_{j} \mid r_{k}\right)=P_{i}\left(m_{j}\right)$ for all $j$ and all $k$ values. Thus, $P_{i}\left(m_{j} \mid r_{k}\right)=P_{i}\left(m_{j}\right) P_{i}\left(r_{k}\right)$.

(II) if we divide the area-source into increments of area with equal size, all the area-source increments have the same earthquake frequency or equal probability of producing an earthquake. Therefore, instead of finding the probability density function $f_{i}\left(r_{k}\right)$, we can use the probability density function related to the distance of area-source increments to the site or $f_{i}\left(r_{h}\right)$, where $r_{h}$ is the distance of area-source increment to the site. Let's assume that there are $n s$ different distances $h=1,2,3, \ldots n s$ for area-increments to the site. Hence, in the area-source approach, the exceedance rate of $\psi^{*}$ due to all sources is:

$$
\begin{aligned}
\Lambda\left(\psi>\psi^{*}\right)= & \sum_{i=1}^{n_{\mathcal{F}}} \lambda_{i}\left[\sum_{j=1}^{n m_{i}} \sum_{h=1}^{n s_{i}} P_{i}\left(\psi>\psi^{*} \mid m_{j}, r_{h}\right) P_{i}\left(m_{j}\right) P\left(r_{h}\right)\right] \\
& =\sum_{i=1}^{n_{\mathcal{F}}} \lambda_{i} \iint P_{i}\left(\psi>\psi^{*} \mid m, r\right) f_{i}(m) f_{i}(r) d m d r .
\end{aligned}
$$

where $f_{i}(r)$ is the continuous probability density function for the area-source distance to the site and $f_{i}(m)$ is the continuous form of applied magnitude-frequency law. In an area-source approach, it is assumed that $\lambda$ is spatially uniform. A likewise assumption is usually applied in the time domain. However, some studies consider estimating $\lambda$ for multiple time intervals with alike length of $T^{*}$ that are smaller than usual (few weeks or few months) and evaluate the changes in the associated $\Lambda$ (Convertito \& Zollo, 2011; Convertito et al., 2012). These types of calculations aim to monitor the influence of some temporary changes in the process of earthquake generation (e.g., fluid or magma intrusions into the rocks pore). Although, such approaches use spatiotemporaly uniform $\lambda$ for each time interval $T^{*}$, they provide a sort of time-dependent $\lambda$ estimation and lead to a time-dependent probabilistic seismic hazard. 
In turn, the zone-less approach does not divide the seismic zone into area-sources with uniform seismic characteristics. Instead, it regards the actual distribution of earthquakes which is neither homogeneous in space nor in magnitude-frequency. In another words, each increment of area (with size of $d A$ ) presents different earthquake frequency (number of earthquakes in unit of time), and the magnitude distribution of earthquakes can also vary from one increment of area to another. Therefore the probability density function for the magnitude-frequency $f\left(m_{j}\right)$, is not independent of the distance $r_{k}$. Moreover, the probability density function for the earthquake-to-site distance $f\left(r_{k}\right)$ is not the same as the probability density function for the area-increment-to-site distance $f_{i}\left(r_{h}\right)$. This means that $\dot{n}\left(m_{j}, r_{h}\right)$ can vary from one location to another, even they have a same distance $r_{h}$ to the site. Indeed, this approach requires calculation of $\dot{n}(m, x, y)$ for each magnitude increment and for all $n a$ area-increments (with centres at $\left(x_{l}, y_{l}\right)$, where $l=1,2,3$, ... na) that construct the zone. Such calculation is more intensive in terms of computation time. The implementation of achieved $\dot{n}\left(m_{j}, x_{l}, y_{l}\right)$ in the relation (1.6) will yield:

$$
\Lambda\left(\psi>\psi^{*}\right)=\sum_{j=1}^{n m} \sum_{l=1}^{n a} P\left(\Psi_{j l}>\psi^{*}\right) \dot{n}\left(m_{j}, x_{l}, y_{l}\right) .
$$

The methodology for estimating $\dot{n}(m, x, y)$ over the zone, implies an location-dependent approach for modelling the earthquake rate $\lambda$, which is described in section 2.4. In chapter 6 , the zone-less method is used for estimation of $\Lambda$ due to an earthquake sequence. Figure 1.4 shows an example of inhomogeneous earthquake density $\operatorname{rates}^{2} \dot{\rho}(m, x, y)$ for the surface projection of subduction interface seismicity between 1992 and 2018 in the Papanoa zone (blue rectangle in Figure 1a of chapter 6). The inhomogeneous spatial distribution of earthquake density rates is different for each magnitude increments as well. Moreover, in this study, the $\dot{n}(m, x, y)$ is estimated for different time intervals and incorporated separately into the equation (1.9) in order to provide a time-dependent estimation of $\Lambda$ with respect to the occurrence date of the 2014 Papanoa thrust earthquake.

Eventually, the earthquake rate $\lambda$ plays an important role in the PSHA and its spatiotemporal variations can implicate changes in the ground motion exceedance rate $\Lambda$.

\footnotetext{
${ }^{2}$ Density rate: number of earthquakes per unit of time and per unit of area
} 


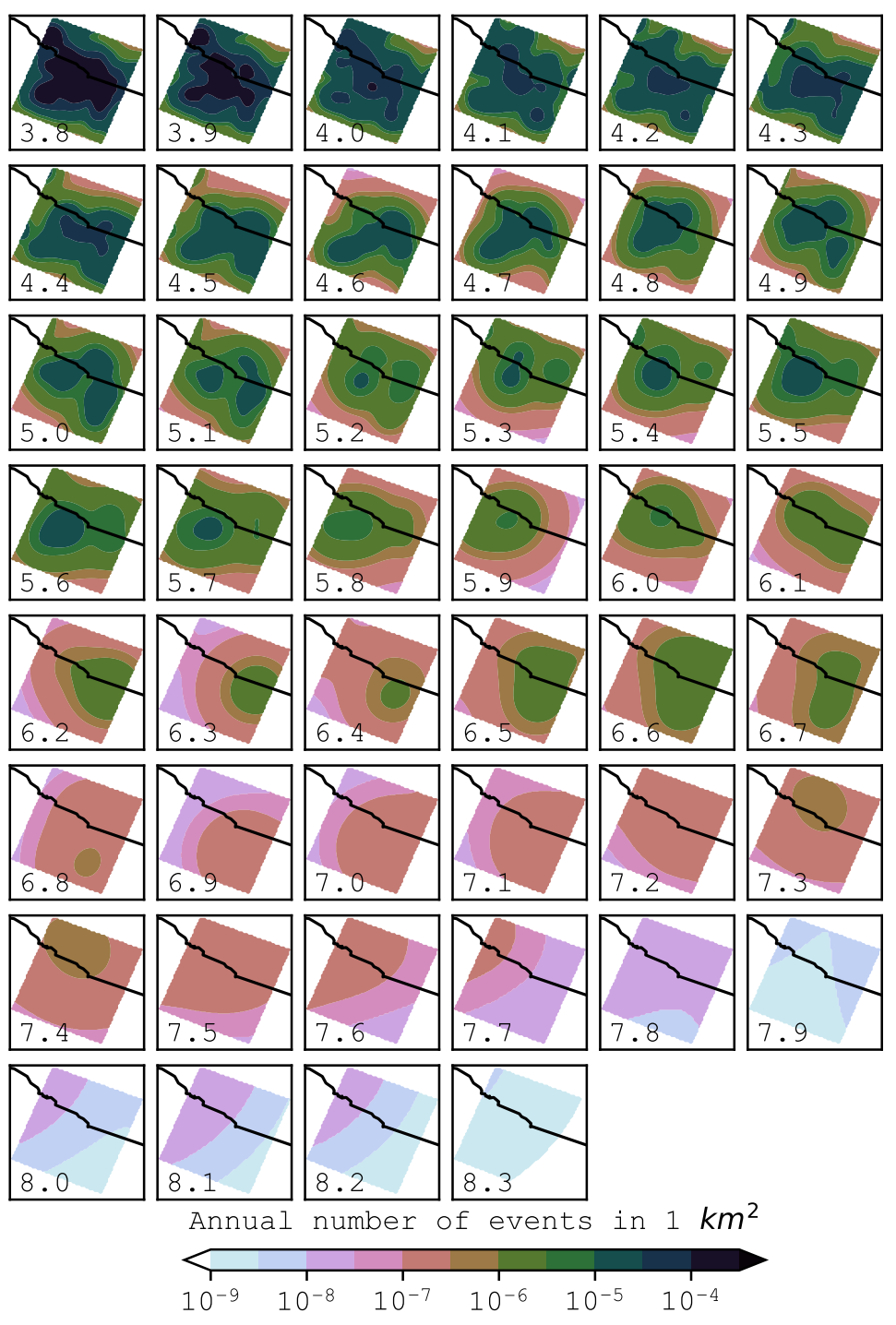

Figure 1.4: The surface projection of the density rate of subduction earthquakes for magnitude increments within $m \in[3.8,8.2]$, and between 1992 and 2018 in the researched zone of chapter 6.

\subsection{Research context}

\subsubsection{Swarm-type sequence}

\section{The 2012-2013 Torreperogil-Sabiote sequence, southern Spain (chapter 3) :}

The only swarm sequence that I study in this dissertation presents a relatively long-lasting earthquake activity that started on $20^{\text {th }}$ October 2012 in Jaén, southern Spain, and smoothed out after more than 8 months. This sequence is located close to the towns of Torreperogil and Sabiote and its largest event with magnitude 3.9 on $5^{\text {th }}$ February 2013 caused slight damage and raised a high social concern and intense debate about the origin of the swarm (Peláez et al., 2013). However, some slight and low magnitude earthquake activity have been started around 2010 in the nearby area.

The tectonic setting of the area is controlled by the the convergence of the Iberia micro-plate 
and the African tectonic plate, which accommodates a very broad deformation zone that hosts the seismic activity in southern Spain (Benito \& Gaspar-Escribano, 2007), including swarm sequences (Martínez-Díaz et al., 2014). Indeed, there have been other strong swarm activities (e.g., April 1998, Iznajar, southern Spain; Carmona et al., 2009), revealing NS strike-slip parallel faulting and complex local deformations, but the 2012-2013 Torreperogil-Sabiote sequence is located at the eastern side of the Guadalquivir Basin, where is known of a very low seismic activity so far (Cantavella et al., 2013). Morales et al. (2015) study the spatial distribution of this sequence using a database of around 7000 earthquakes $(-0.8<M<3.9)$ from October 2012 to October 2013, which is recorded by local, temporary and also national seismic stations. Their improved hypocentral locations show three separate clusters, where two of them (the western and the central clusters) show a NS trend and depths of 4 to $6.5 \mathrm{~km}$, and one (the eastern cluster) presents a ESE-WSW orientation with a depths ranging from 2 to $7.5 \mathrm{~km}$. Thus, Morales et al. (2015) propose the presence of previously unknown NS strike-slip blind faults and suggest that this swarm sequence is associated with this highly deformed region due to bending. They also find that the central cluster with a steep west-dipping plane mostly represents the high activity period of the sequence since December 2012 until April 2013 and it includes the largest event with magnitude 3.9 .

In chapter 3, I apply the statistical approach for characterizing this swarm activity in the time domain. Nevertheless, I am not fortunate to use the comprehensive database analysed by Morales et al. (2015), and I carry out the statistical analysis using a database of $>2000$ earthquakes with magnitudes $-0.1<M<3.9$, provided by Geographic Institute of Spain (IGN). I divide the earthquake activity into three phases of pre-, main- and post-activity to better understand the evolution of earthquake rates during time. The addressed questions in this research are: Which are the characteristics of this sudden rise in the earthquake frequencies? How does the magnitude distribution change? Is the aseismic rate of earthquakes experiencing a new state during the main-activity phase, or it is just revealing a pre-established nature which was not well-known to us? What would be the source of any likely changes in rate of the aseismic loading? What can we learn from the tail of this swarm (the post-activity phase; since mid-2013 to the end of 2014)?

\subsubsection{Mainshock-aftershock sequence; crustal faulting}

I study the two following earthquake series that are related to shallow continental crust faulting and are categorized as mainshock-aftershock sequences:

\section{The 2012 Ahar-Varzeghan sequence, north-western Iran (chapter 4) :}

This sequences is associated to an earthquake doublet in the shallow crust of north-western Iran, between the towns of Ahar and Varzeghan. This type of intraplate doublets are not as common as they are in subduction zones (Kagan \& Jackson, 1999). The 2012 Ahar-Varzeghan doublet comprises two events with magnitudes of $M_{\mathrm{w}} 6.4$ and $M_{\mathrm{w}} 6.2$. They occurred on $11^{\text {th }}$ August 2012, with $6 \mathrm{~km}$ and 11 minutes separation in epicentral location and origin time, respectively. They ruptured distinct and previously unknown fault structures and they produced an EW oriented surface rupture of $\sim 13.5 \mathrm{~km}$ length, and caused extensive damage and losses (over 300 deaths and 3000 injuries) in the nearby towns (Copley et al., 2013).

The earthquakes in north-western Iran are among the most destructive and hazardous events 
in the country and they are historically associated with the North Tabriz Fault, which has a length of $>150 \mathrm{~km}$ and NW-SE orientation. This fault is located at almost $50 \mathrm{~km}$ to the south from the mentioned doublet. The kinematics of this fault predominantly show right-lateral strike-slip motion, which is an outcome of the convergence between the Arabian and the Eurasia tectonic plates that accommodates the ongoing deformation in Anatolia and north-western Iran (Reilinger et al., 2006). The Ahar-Varzeghan doublet is located in an area characterized by a low deformation rate and lack of significant seismic activity prior to the consequent earthquake sequence (Djamour et al., 2011). However, recent geomorphological measurements show a continental lithosphere deformation which was previously undetected, suggesting that this region should not be considered a rigid block any more (Copley et al., 2013).

In chapter 4, the 2012 Ahar-Varzeghan earthquake sequence is analysed through statistical and coseismic static stress modelling. The addressed research questions are: What is the nature of this seismic activity? Can we claim that the first shock triggered the second shock? How could both shocks explain the spatial distribution of the aftershocks?

\section{The 2001 Coyuca sequence, southern Guerrero, Mexico (chapter 4) :}

This sequence started with a moderate mainshock with magnitude $M_{\mathrm{w}} 5.8$ with a normal faulting mechanism in the shallow crust, located at the south of Guerrero (Mexico) and close to the town of Coyucade Benítez. The focal mechanism solutions for this mainshock and its four largest aftershocks show nodal planes with ENE-WSW to ESE-WNW orientations, with almost pure normal slip over an oblique plane. Iglesias et al. (2004) and Zhang et al. (2009) favour south dipping plane based on the hypocentral distribution of re-located aftershocks. The earthquakes hypocentres are situated at depths less than $15 \mathrm{~km}$, above a subduction area with no large megathrust earthquake since 1911.

Coyuca is located in an area that scantly presents subduction seismicity, which is called the seismic gap of Guerrero. This gap is known to be associated with a nearly four-year periodic downward motion of oceanic Cocos plate beneath the continental North American plate, where the accumulated tectonic stress is being partially released via the slow-slip events each $\sim$ four years (Kostoglodov et al., 2003; Radiguet et al., 2016). The time series data from the global positioning system (GPS) in the continental crust of the North American plate, exhibits a northward motion with a roughly constant rate along the North American trench and during periods between consecutive slow-slip events. On the contrary, the southward movement of the North American crust during the slow-slip events, reveals a greater release of the accumulated strain (up to $75 \%$ ) within the seismic gap section (Radiguet et al., 2012). Indeed, the location of the 2001 Coyuca earthquake sequence is almost above a zone of low to moderate coupling between the Cocos oceanic crust and the North American crust, and the beginning of this sequence almost coincides with the beginning of the 2001-2002 slow-slip event in the Guerrero.

In chapter 5, I apply physic-based static stress modelling to evaluate how the strain accumulation/release, could have triggered this earthquake sequence. It is worth mentioning that I could not carry out a reliable statistical analyses because the catalog data related to this sequence suffers from systematic damage (i.e., broken hard disc containing digital data) and discontinuous coverage in time (i.e., absence of seismic records during periods of one days and few weeks) which highly misinterpret the results of any statistical modelling. However, I address the following questions: What is the triggering influence of strain accumulation during inter-slow-slip 
period (sine mid-1998) on the Coyuca sequence? What about the triggering influence of the coeval slow-slip event in 2001? Do the positively charged areas by the static stress agree with the aftershock distribution?

\subsubsection{Subduction sequence; large thrust earthquake}

\section{The 2014 Papanoa sequence, southern Guerrero,Mexico (chapter 6) :}

I study the 2014 Papanoa subduction earthquake sequence that is also a mainshock-aftershock sequence. It is located nearby the coast of Guerrero (Mexico), between towns of Papanoa and Zihuatanejo. This sequence began with a large thrust earthquake on $18^{\text {th }}$ April 2014 with $M_{\mathrm{w}} 7.3$ at a hypocentral depth of $15 \mathrm{~km}$ and was followed by two large aftershocks of moment magnitudes 6.6 and 6.2 on $8^{\text {th }}$ and $10^{\text {th }}$ May 2014, respectively. All three events caused huge damage near the epicentre, along the coast and inland. They were strongly felt in Mexico City, several walls collapsed and the city suffered from partial power outage and general panic.

The mainshock ruptures the subduction interface, where the Cocos oceanic plate is forced underneath the continental North American plate (UNAM Seismology Group, 2015). At shallow depths, where the Papanoa sequence occurred, in addition to megathrust earthquakes and seismic process of earthquake-earthquake triggering, an aseismic process of slow-slip events is also detected along the subduction interface (Kostoglodov et al., 2003). The megathrust earthquakes and the slow-slip events occur along interplate adjacent regions, and indeed might interact. The $18^{\text {th }}$ April 2014 earthquake have ruptured an area that very probably has experienced other megathrust ruptures during history; like the $14^{\text {th }}$ March 1979 Petatlán earthquake with $M_{\mathrm{w}} 7.4$ (Mendoza \& Martínez López, 2017). It is proposed that this interplate area shares adjacent seismic asperities with the edge of slow-slip region of low coupling, and this might influence the weakening of earthquake nucleation close to the hypocentre of Papanoa earthquake (Radiguet et al., 2016). The same study also notices that the 2014 slow-slip event in Guerrero has just started about two months before, in February 2014, and by April 2014 it had already released an energy equivalent to a $M_{\mathrm{w}} 7.1$, that could have triggered the Papanoa mainshock.

To investigate the seismic and aseismic activity along the subduction interface in this seismogenic zone, I selected events that could be associated to this interface. Thus, all shallow clusters related to intraplate continental crust are discarded and events with depth $<45 \mathrm{~km}$, and within a vertical distance of $\pm 5 \mathrm{~km}$ to a 3D subduction interface of $10 \mathrm{~km}$ thickness, are analysed within different time frames in chapter 6 . The addressed questions are: What is the nature of the loading force during this sequence? How much is the contribution of aftershocks along the subduction interface? Does the sequence influence the spatiotemporal density and rate of earthquakes? If so, what is the result of implementing these updated earthquake densities and rates in the ground motion exceedance rate? 


\section{Chapter 2}

\section{Methodology}

\subsection{Statistical approach}

An earthquake is produced by a rupture over a finite area (of few to hundreds of $\mathrm{km}^{2}$ ) in the Earth's interior. This process takes place during a finite time interval, which can last for a few seconds to several minutes. In turn, when an earthquake is considered as a member of a greater population that is distributed in time and space domains, it is defined by three punctual parameters: origin time, hypocentre and magnitude. In such scales, the earthquake population is often formulated into empirical laws, which describe the distribution of earthquake parameters. Statistical seismology avails these laws for modelling earthquake sequence by assuming that earthquakes are points within a point process. In a point process, the points (i.e. earthquakes) are random objects that can take values of a countable subset in a given spatiotemporal domain. There might be an assigned size to these points with values within a finite range. In the case of an earthquake process, this size is the earthquake magnitude. Being random in time and space domains, does not mean the process of earthquake generation is patternless. The stochastic modelling uses probabilistic rules for predicting the occurrence patterns of earthquakes in time and space domains.

In the following sections, some common probability distributions of the earthquake parameters are introduced. Firstly, the most commonly used magnitude-frequency modelling is explained. Then, the basic mathematical concepts of a one-dimensional (time domain) point process, such as hazard function and conditional intensity, are described. Then, some probability distributions for earthquake epicentral location (space domain) and their integration into the spatiotemporal modelling of earthquake sequences are presented and discussed.

\subsubsection{Magnitude-frequency distribution}

One of the fundamental earthquake scaling relationships is the magnitude-frequency distribution. During a given period of time and within a seismogenic zone, the magnitude of an earthquake can be considered a random object where its frequency decreases exponentially with its size. The mathematical models that relate the size of an earthquake and the frequency of occurrence 
for that size in a determined seismic source are called recurrence models.

Gutenberg \& Richter (1944) studied the earthquake catalog of southern California over a period of about 40 years (since the beginning of $20^{\text {th }}$ century) and showed that the occurrence rate of earthquakes with magnitude greater than or equal to $m$, can be expressed by the equation (2.1) that is known as the standard Gutenberg-Richter (GR) law:

$$
\dot{\mathcal{N}}^{G R}(m)=10^{a-b m}
$$

where $\dot{\mathcal{N}}(m)$ is the observed cumulative number of events with magnitude $\geq m$ in unit of time and the superscript $G R$ appears when this number is associated to the prediction by the Gutenberg-Richter law. Parameters $a$ and $b$ are constant and positive values. This law covers an infinite range of magnitudes and it is the most commonly applied recurrence model. With respect to this law, the occurrence rate for magnitude $M$ (where $m \leq M<m+\Delta m$ for small $\Delta m)$ or $\dot{n}^{G R}(M)$ is:

$$
\dot{n}^{G R}(M)=\left.\frac{\dot{\mathcal{N}}^{G R}(m)-\dot{\mathcal{N}}^{G R}(m+\Delta m)}{\Delta m}\right|_{m=M} \approx-\left.\frac{d}{d m} \dot{\mathcal{N}}^{G R}(m)\right|_{m=M}=b \ln (10) 10^{a-b M} .
$$

The magnitude range of earthquakes within a seismogenic zone is finite (Bommer et al., 2007). Thus, a more realistic application of this model should consider a lower and an upper bounding values. The lower bounding value (or the cut-off magnitude) is usually equal or greater than the magnitude of completeness $m_{c}$. The $m_{c}$ refers to the lowest magnitude at which $100 \%$ of the earthquakes are detected in space and time domains. A correct estimate of $m_{c}$ is important because if we take a larger value, we loose usable data and if take a lower value it leads to bias in modelling due to the incompleteness of data (Mignan \& Woessner, 2012). Thus, the minimum cut-off magnitude equal to $m_{c}$ is usually applied for the estimation of the GR parameters $a$ and $b$ that determine the seismicity rate. The principal method for estimation of the GR parameters is introduced in Box 2.1. Additionally, the relation (2.1) predicts nonzero mean rates of exceedance for magnitudes up to infinity, which is not real. Therefore, as the upper bound value, some largest possible earthquake or magnitude $m_{\max }$ needs to be associated with every source zone (Kramer, 1996; Kijko, 2004). For such double-truncated form of GR law, the total number of events in unit of time is $\dot{N}^{G R}=\dot{\mathcal{N}}^{G R}\left(m_{c}\right)-\dot{\mathcal{N}}^{G R}\left(m_{\max }\right)$. Thus, the probability of exceedance for magnitude $m$ will be:

$$
P(M \geq m)=\frac{\dot{\mathcal{N}}^{G R}(m)-\dot{\mathcal{N}}^{G R}\left(m_{\max }\right)}{\dot{N}^{G R}}=\frac{10^{(a-b m)}-\dot{\mathcal{N}}^{G R}\left(m_{\max }\right)}{\dot{N}^{G R}}
$$

and the cumulative distribution function $F(m)$, and the probability density function $f(m)$, respectively are:

$$
\begin{gathered}
F(m)=1-P(M \geq m)=1-\frac{10^{(a-b m)}-\dot{\mathcal{N}}^{G R}\left(m_{\max }\right)}{\dot{N}^{G R}} \\
f(m)=\frac{d}{d m} F(m)=\frac{b \ln (10) 10^{a-b m}}{\dot{N}^{G R}}=\frac{\dot{n}^{G R}(m)}{\dot{N}^{G R}}
\end{gathered}
$$


The most commonly applied form of the GR law for analysing the earthquake catalogs considers only the lower magnitude limit, where $\dot{N}^{G R}=\dot{\mathcal{N}}^{G R}\left(m_{c}\right)$, and:

$$
f(m)=\frac{b \ln (10) 10^{a-b m}}{10^{a-b m_{c}}}=b \ln (10) 10^{b\left(m-m_{c}\right)} .
$$

The $m_{c}$ associated to a fix time interval $T$ (e.g., the time period of an earthquake catalog or an earthquake sequence) represents the minimum magnitude value, for which all earthquakes with magnitudes $\geq m_{c}$ within any continuous subset of $T$ are fully recorded.

In modelling the magnitude-frequency distribution, as it is shown in the equation (2.6), events with magnitudes lower than $m_{c}$ are excluded, despite the fact that some continuous subsets $t^{*} \subset T$ might have a smaller completeness magnitude $m_{c}^{*}<m_{c}$. Indeed, thanks to the enhancements in the sensibility of the seismic instruments and the better coverage and expansion of seismic networks during the recent decades, the lower limit for fully detected magnitudes has been improved (i.e., $m_{c}$ progressively takes lower values). Thus, the completeness magnitude associated to more recent time periods are usually smaller than a general $m_{c}$ estimated for a catalog with a longer duration. Likewise, during the occurrence of an earthquake sequence in a time interval $T$, sometimes the data become collected with the help of additional temporary, local seismic stations which also results in a non-uniform $m_{c}$ value detection during the earthquake sequence period $T$.

Weichert (1980) considers the variation of completeness magnitude within different periods (subsets of $T$ ) to include more events in the estimation of the GR parameters. Let's consider that an earthquake sequence consists of events with magnitudes of range $\left[m_{0}, m_{\max }\right]$, where $m_{0} \leq m_{c}$, and we discretize this magnitude range into $J$ classes $\mathbb{M}_{J}=\left\{m_{1}, m_{2}, \ldots, m_{J}\right\}$. Assuming that each class $m_{j} \in \mathbb{M}_{J}$ is completely recorded during a given continuous time interval $t_{m_{j}} \subseteq T$, we can find the number of repeats of all magnitude classes within their associated $t_{m_{i}}$ or $\mathbb{N}_{J}=\left\{n_{m_{1}}, n_{m_{2}}, \ldots, n_{m_{J}}\right\}$. For some $m \in \mathbb{M}_{J}$ with $n_{m}=0$, the probability of observing an event with this magnitude is zero: $P_{\text {obs }}(m)=0$ but if $n_{m} \neq 0$ this probability will be:

$$
P_{o b s}(m)=\frac{n_{m}}{\sum_{j=1}^{J} n_{m_{j}}}=\frac{\dot{n}_{m} t_{m}}{\sum_{j=1}^{J}\left(\dot{n}_{m_{j}} t_{m_{j}}\right)},
$$

where $\dot{n}_{m}$ is the observed number of events with magnitude $m \in \mathbb{M}_{J}$ during $t_{m}$ and in time unit. Applying the GR law implies that all the magnitude classes $m \in \mathbb{M}_{J}$ inside $\left[m_{0}, m_{\text {max }}\right]$ have a nonzero rate of $\dot{n}_{m}^{G R}=\dot{N}^{G R} f(m)$ and a nonzero probability of $P(m)$ that can be given by:

$$
P(m)=\frac{\dot{n}_{m}^{G R} t_{m}}{\sum_{j=1}^{J}\left(\dot{n}_{m_{j}}^{G R} t_{m_{j}}\right)}=\frac{\dot{N}^{G R} f(m) t_{m}}{\sum_{j=1}^{J}\left(\dot{N}^{G R} f\left(m_{j}\right) t_{m_{j}}\right)}=\frac{10^{-b m} t_{m}}{\sum_{j=1}^{J}\left(10^{-b m_{j}} t_{m_{j}}\right)} .
$$

The incorporation of completeness time interval $t_{m}$ for each magnitude class $m$, helps us finding a more statistically reliable value for $P(m)$ which is essential to the estimation of likelihood function $^{1}$ for finding the best GR parameters (see Box 2.1).

\footnotetext{
${ }^{1}$ The function that presents the probability of our observations being ruled by a given law. In other words, the probability that an observed distribution obeys an empirical law or model (with a determined parameters) can be estimated using the likelihood function or L(parameters I observations).
} 


\section{Box 2.1}

\section{Gutenberg-Richter parameters estimation}

The probability for an observed magnitude distribution to follow the Gutenberg-Richter law (with a determined $a$ and $b$ ) can be estimated using the likelihood function or $L\left(a, b \mid \mathbb{N}_{J}, \mathbb{M}_{J}\right)$. It is important to notice that the GR law models the distribution of magnitudes regardless of their occurrence order. In other words, all the combinations that create $\mathbb{N}_{J}$ set, should be considered in the likelihood function $L$. The probability for just one $\mathbb{N}_{J}$ set to happen, is $\prod_{i=1}^{J} P\left(m_{i}\right)^{n_{m_{i}}}$. Therefore, the likelihood function $L$ will be:

$$
\begin{gathered}
L\left(a, b \mid \mathbb{N}_{J}, \mathbb{M}_{J}\right)=\left(\begin{array}{c}
N \\
n_{m_{1}}
\end{array}\right)\left(\begin{array}{c}
N-n_{m_{1}} \\
n_{m_{2}}
\end{array}\right) \ldots\left(\begin{array}{c}
n_{m_{J}} \\
n_{m_{J}}
\end{array}\right) \prod_{i=1}^{J} P\left(m_{i}\right)^{n_{m_{i}}} \\
=\frac{N !}{\prod_{i=1}^{J} n_{m_{i}} !} \prod_{i=1}^{J} P\left(m_{i}\right)^{n_{m_{i}}},
\end{gathered}
$$

where $N=\sum_{i=1}^{J} n_{m_{i}}$.

The best $b$-value that maximizes the likelihood function $L$, or equivalently its logarithm or $L L$ (log-likelihood function) represents the best fit between the model and the observations. Placing the equation (2.8) into the logarithm of equation (B.1) gives:

$$
\begin{aligned}
L L\left(a, b \mid \mathbb{N}_{J}, \mathbb{M}_{J}\right)= & \log (N !)-\sum_{i=1}^{J} \log \left(n_{m_{i}} !\right)+\sum_{i=1}^{J} n_{m_{i}} \log \left(t_{m_{i}}\right) \\
& -\sum_{i=1}^{J} n_{m_{i}} b m_{i}-N \log \left(\sum_{j=1}^{J} 10^{-b m_{j}} t_{m_{j}}\right) .
\end{aligned}
$$

The last term in equation (B.2) does include $n_{m}$, and therefore the summation will count for all the magnitude classes $m$ inside $\left[m_{0}, m_{\max }\right]$, even if $n_{m}=0$. The relation (B.2) becomes maximum if $\frac{\partial}{\partial b} L L=0$ and $\frac{\partial^{2}}{\partial b^{2}} L L<0$. These conditions for maximizing the logarithm of likelihood function or $L L$, imply the two equations given by Weichert (1980):

$$
\begin{gathered}
\frac{\sum_{i=1}^{J} n_{m_{i}} m_{i}}{N}=\frac{\sum_{i=1}^{J} t_{m_{i}} m_{i} 10^{-b m_{i}}}{\sum_{i=1}^{J} t_{m_{i}} 10^{-b m_{i}}} \\
N\left[\frac{\left(\sum_{i=1}^{J} t_{m_{i}} m_{i} 10^{-b m_{i}}\right)^{2}}{\left(\sum_{i=1}^{J} t_{m_{i}} 10^{-b m_{i}}\right)^{2}}-\frac{\sum_{i=1}^{J} t_{m_{i}} m_{i}^{2} 10^{-b m_{i}}}{\sum_{i=1}^{J} t_{m_{i}} 10^{-b m_{i}}}\right]<0,
\end{gathered}
$$

where $j$ is replaced by $i$. Finding the $b$-value that complies the above equations is usually carried out using numerical estimation by starting from a minimum guess like $b=0.5$ and rising it gradually. Once the $b$-value is estimated, the $a$-value can be calculated using equation (2.1) and replacing $m$ by $m_{0}$ and $\dot{\mathcal{N}}^{G R}(m)$ by $\dot{\mathcal{N}}\left(m_{0}\right)$. Therefore: $a=\log \left(\dot{\mathcal{N}}\left(m_{0}\right)\right)+b m_{0}$. 
Figure 2.1 shows an example of applying GR law to find the magnitude-frequency distribution of earthquakes in the catalog of southern Spain between 1048 and 2011 (Figure 2.2). The applied catalog is obtained by IGN \& UPM $(2013)^{2}$, where the cut-off magnitude of 4.0 is introduced as the best completeness magnitude for area within the map in Figure 2.2. The annual rates of each magnitude are shown in blue triangles and the GR parameters $a$ and $b$ are estimated using the maximum likelihood method described in Box 2.1.
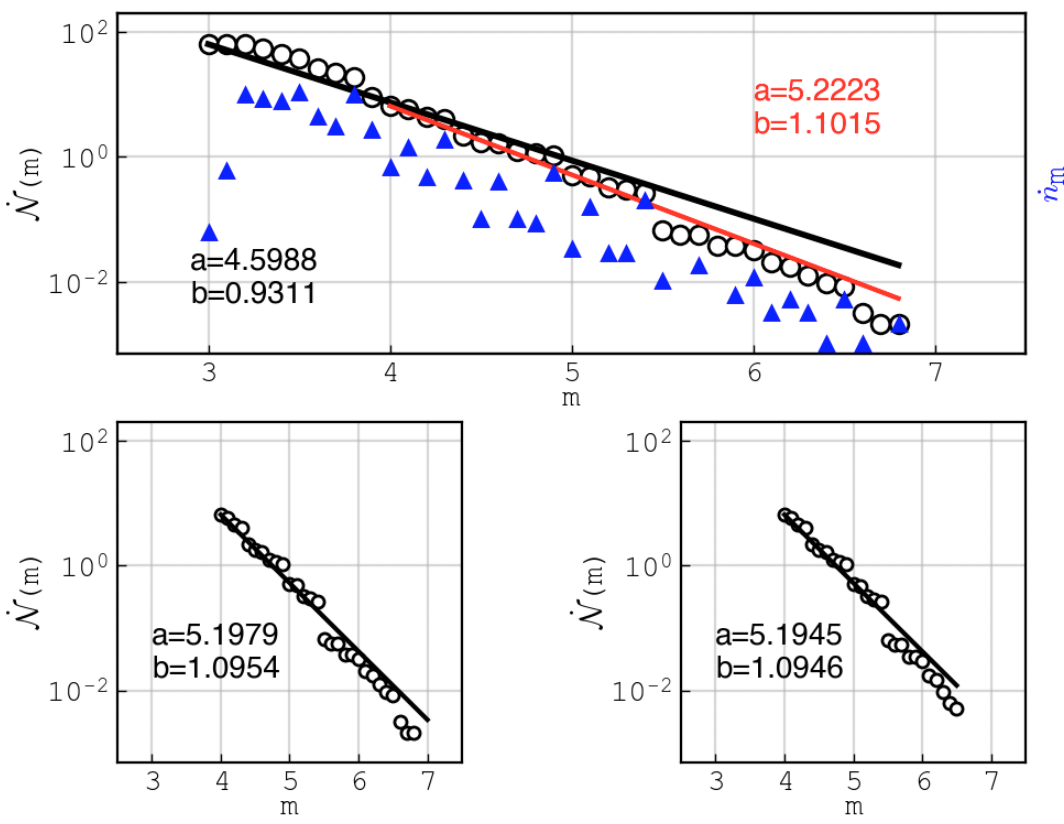

Figure 2.1: Up: the black line shows an example for the standard form of the Gutenberg-Richter law. The $a$-value and $b$-value are calculated using maximum likelihood estimation method (see Box 2.1) for the earthquakes in southern Spain (Figure 2.2) covering magnitudes within the catalog or $[3.0,6.8]$. The red line presents the low-limit truncated Gutenberg-Richter law, where a $m_{c}=4.0$ is applied to the same database. The blue triangles stands for the observed annual number of events $\dot{n}_{m}$ and the empty circles show the annual cumulative number of events $\dot{\mathcal{N}}(m)$. Down: the double-truncated form with two different maximum limits for comparison, where $m_{c}=4.0$ in both estimations : $m_{\max }=7.0$ (left) and $m_{\max }=6.5$ (right).

\section{APPLICATION TOOL 2.1}

\section{An application tool for spatial estimation of Gutenberg-Richter parameters ${ }^{\mathrm{I}}$}

In the following, I describe an application tool that I developed under programming software MATLAB and in order to calculate the GR parameters $a$-value and $b$-value for a $2 \mathrm{D}$ grid of coordinates. This program tool is named abMapper 2 and is accompanied by a user interface as in Figure 2.3, that provides a selection of initial conditions related to the GR parameter estimation.

\footnotetext{
${ }^{2}$ Instituto Geografico Nacional \& Universidad Politécnica de Madrid
} 


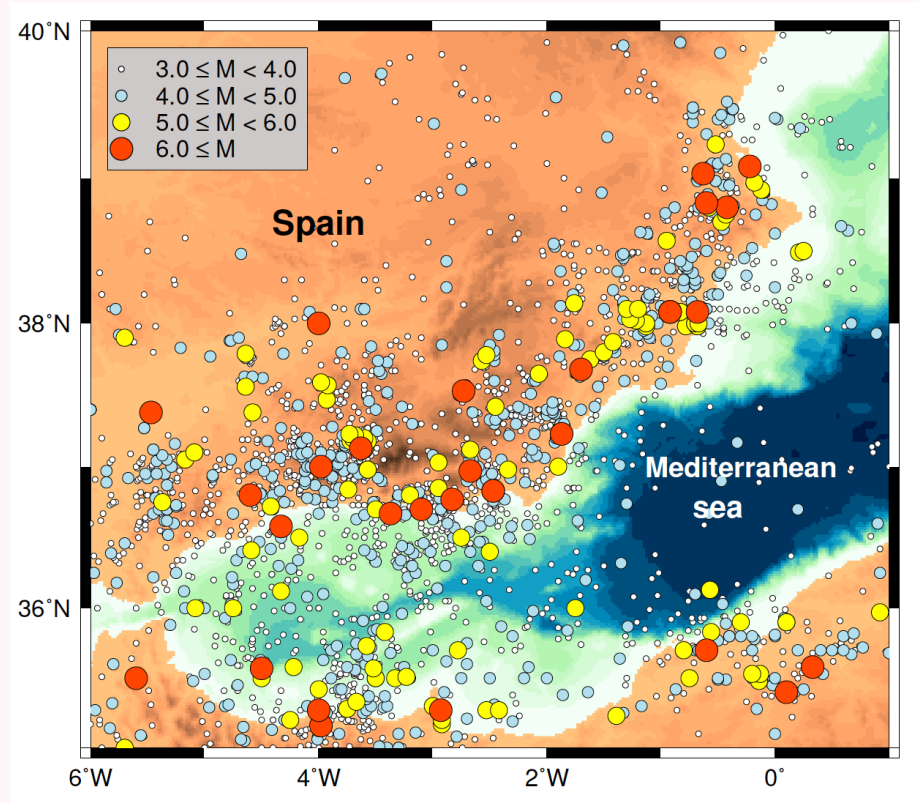

Figure 2.2: Earthquakes with magnitude $M_{\mathrm{w}} \geq 3.0$ in south-eastern of Spain (IGN \& UPM, 2013) between years 1048 and 2011.

The presented examples in this Box are calculated using the earthquake catalog of Spain by IGN \& UPM (2013) for an area within longitudes $\left[-6^{\circ}, 1^{\circ}\right]$ and latitudes $\left[35^{\circ}, 40^{\circ}\right]$ in south-eastern Spain that includes earthquakes from 1048 to 2011 with magnitudes between 3.0 and 6.8 as shown in Figure 2.2).

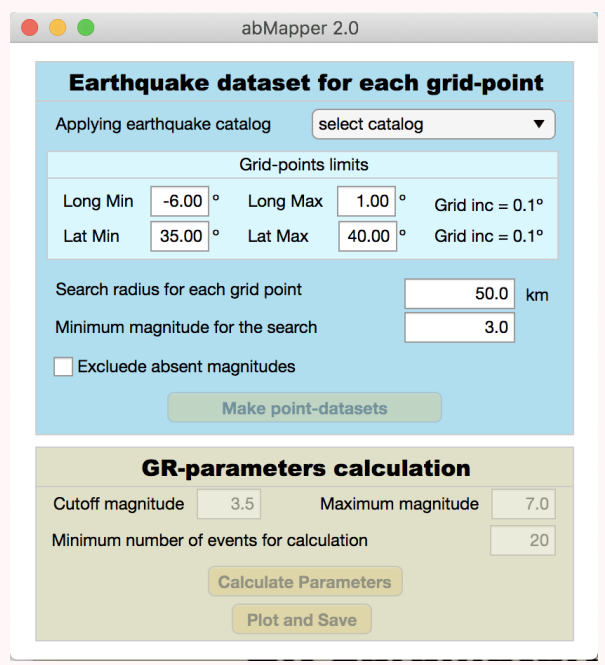

Figure 2.3: The user interface of the designed application tool for spatial estimation of $b$-value.

Firstly, a grid of coordinates with separations of $0.1^{\circ}$ along longitude and latitude is made, so we can further assign a subset of the earthquake catalog to each grid-point. The associated subset includes earthquakes with a given "Minimum magnitude" and within a given spatial distance or search radius to the grid-point. Then the $b$-value related to the dataset of each grid-point is estimated if the minimum number of events with magnitudes $\geq m_{c}$ in that dataset is higher than a given number. The applied method is the 
maximum likelihood estimation (Weichert, 1980). The unit of time is considered to be "one year" and thus, the completeness years $\mathrm{y}_{i}$ for different magnitudes $m_{i}$ of the applied catalog is implemented inside the code to calculate $t_{m_{i}}=2011-\mathrm{y}_{i}$. For magnitudes $<3.5$, $[3.5,3.9],[4.0,4.4],[4.5,4.9],[5.0,5.4][5.5,5.9]$ and $\geq 6.0$, the completeness years of 1978, 1975, 1908, 1883, 1800, 1520 and 1048 are used. Figure 2.4 shows the result for spatial distribution of GR parameters $a$ and $b$, where $m_{c}=4.0$ and $m_{\max }=6.8$ are applied.
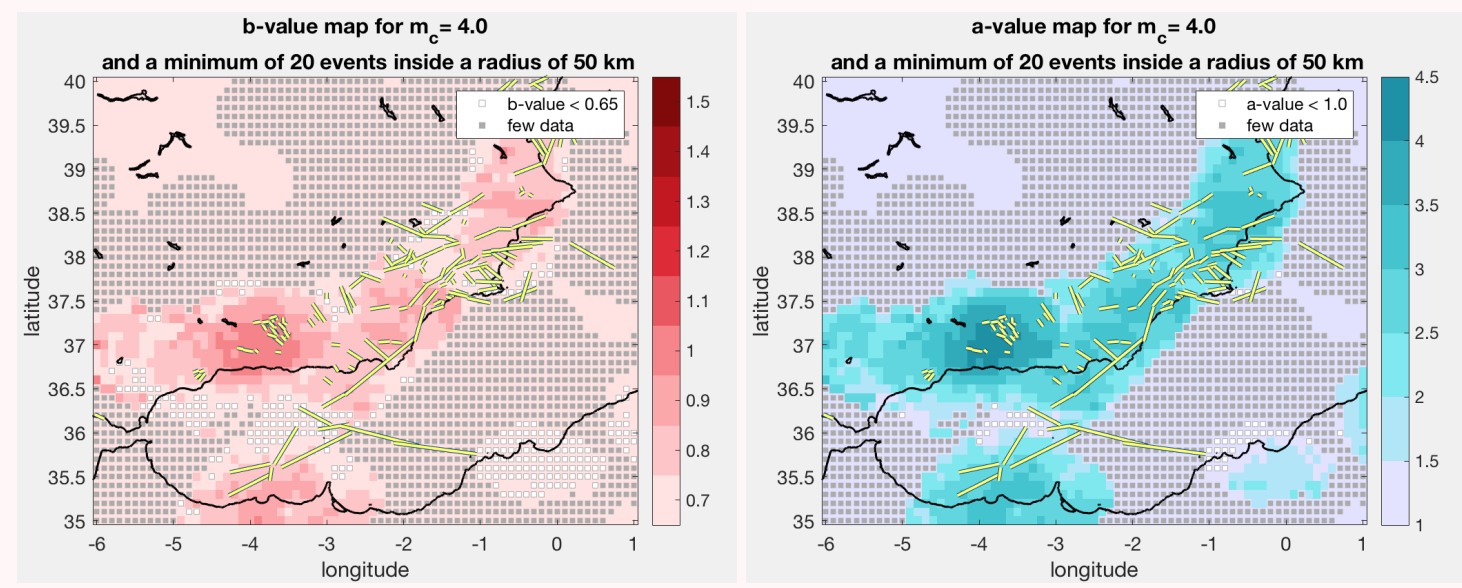

Figure 2.4: The spatial distribution of Gutenberg-Richter parameters.

Another output of the program, which is the annual rate of earthquake per $\mathrm{km}^{2}$ (the density rate of the earthquakes), is also shown in Figure 2.5.

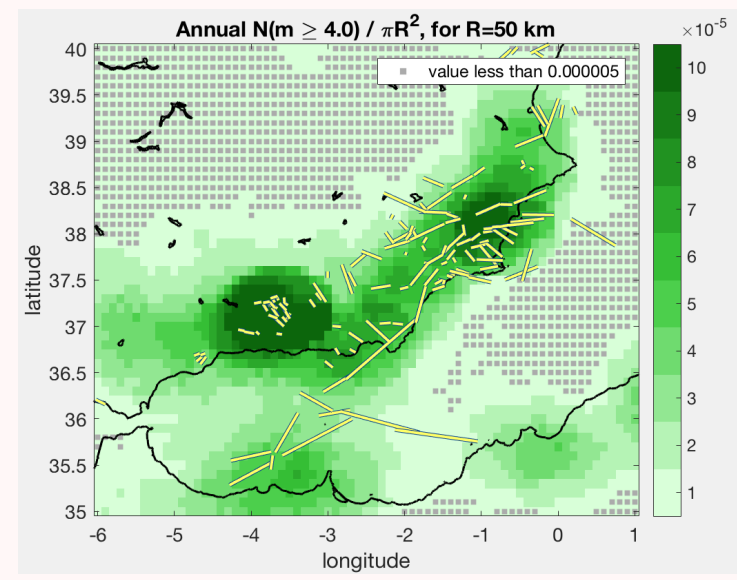

Figure 2.5: The annual number of earthquakes in unit of area $\left(\mathrm{km}^{2}\right)$.

Iavailable in https://gitlab.com/pouyeupm/abMapper2

\subsubsection{Seismicity rate modelling}

In an earthquake process considered as a point process, events inside a finite area $\Phi$ can take random values in a given time range $T=\left[T_{i n i}, T_{\text {end }}\right]$. This implies that the number of events 
$\mathrm{N}(\Delta t)$ within any subset $\Delta t$ of $T$, is a random value as well. The long-term ${ }^{3}$ average of $\mathrm{N}(\Delta t)$ denoted by $\overline{\mathrm{N}}(\Delta t)$, for $Y$ observations $(Y \ggg 1)$ is:

$$
\begin{aligned}
\overline{\mathrm{N}}(\Delta t)= & \frac{n(\mathrm{~N}(\Delta t)=1)+2 n(\mathrm{~N}(\Delta t)=2)+\ldots+N n(\mathrm{~N}(\Delta t)=N)}{Y} \\
= & \frac{n(\mathrm{~N}(\Delta t)=1)}{Y}+2 \frac{n(\mathrm{~N}(\Delta t)=2)}{Y}+\ldots+N \frac{n(\mathrm{~N}(\Delta t)=N)}{Y},
\end{aligned}
$$

where $n(\mathrm{~N}(\Delta t)=k)$ represents the number of observation with " $k$ events in a time interval of size $\Delta t$ ", $N$ is the total number of events in $T$, and $Y=\sum_{k=1}^{N} n(\mathrm{~N}(\Delta t)=k)$. For a very large $Y$ we can write $\frac{n(\mathrm{~N}(\Delta t)=k)}{Y}=P(\mathrm{~N}(\Delta t)=k)$. Hence, the expected number of events $E(\mathrm{~N}(\Delta t))$ in an interval of size $\Delta t \subset\left[T_{i n i}, T_{\text {end }}\right]$ and the long-term average of number of events in $\Delta t$ are equal:

$$
E(\mathrm{~N}(\Delta t))=P(\mathrm{~N}(\Delta t)=1)+2 P(\mathrm{~N}(\Delta t)=2)+\ldots=\overline{\mathrm{N}}(\Delta t) .
$$

For time unit, the long-term average of number of events is $\lambda=\frac{\overline{\mathbb{N}}(\Delta t)}{\Delta t}$ which defines the earthquake rate or long-term average of the number of events in unit of time and it is usually denoted by $\lambda$. Therefore :

$$
E(\mathrm{~N}(\Delta t))=\lambda \Delta t
$$

The earthquake rate $\lambda$, can be time-dependent (see page 23) or not (see page 24), though in this dissertation mainly a time-dependent $\lambda$ is considered for seismicity rate modelling. Before introducing the applied models, I explain the mathematical concept of hazard function ${ }^{4}$ which is essential for understanding further formulations involved in the earthquake rate modelling.

\section{Hazard function}

During a point process $Z$, and at a certain time $t_{0}$, we are interested to know the time of the next event $e_{\text {next }}$. If $F_{t_{0}}(\tau)$ is the cumulative distribution function for the waiting time $\tau$ (since $t_{0}$ ) until the occurrence of $e_{\text {next }}$, then $F_{t_{0}}(\tau+\Delta \tau)-F_{t_{0}}(\tau)$ gives the probability that $e_{\text {next }}$ takes place within time interval $(\tau, \tau+\Delta \tau]$. For very small values of $\Delta \tau$ we will have:

$$
\lim _{\Delta \tau \rightarrow 0} F_{t_{0}}(\tau+\Delta \tau)-F_{t_{0}}(\tau)=d F_{t_{0}}(\tau)=f_{t_{0}}(\tau) d \tau
$$

where, $f_{t_{0}}(\tau) d \tau$ is the probability that $e_{n e x t}$, occurs between $t_{0}+\tau$ and $t_{0}+\tau+d \tau$, and $f_{t_{0}}(\tau)$ is the probability density function for the random variable $\tau$, thus:

$$
\int_{0}^{\infty} f_{t_{0}}(\tau) d \tau=1
$$

In a point process we assume that $e_{n e x t}$ is in existence. This means that $e_{\text {next }}$ will occur anyway and we are not including any death probability ${ }^{5}$ for this event. Therefore, if time passes and $e_{\text {next }}$ does not happen, the probability of its occurrence grows, even if the value of probability density decreases for bigger $\tau$. This assumption that $e_{\text {next }}$ will happen sooner or later, leads to a conditional probability for the waiting time $\tau$. Indeed, the probability that next event $e_{\text {next }}$,

\footnotetext{
${ }^{3}$ The average over many observations (and not the average over a long time)

${ }^{4}$ The hazard function is not the seismic hazard

${ }^{5}$ The probability of an event not happening
} 
occurs in $\left(t_{0}+\tau, t_{0}+\tau+d \tau\right]$ is conditioned to that it does not occur in $\left(t_{0}, t_{0}+\tau\right]$. This conditional probability is used to define the hazard function or $\mathbf{h}_{t_{0}}(\tau)$ :

$$
\mathbf{h}_{t_{0}}(\tau) d \tau=\frac{f_{t_{0}}(\tau) d \tau}{1-F_{t_{0}}(\tau)}=\frac{f_{t_{0}}(\tau) d \tau}{S_{t_{0}}(\tau)}
$$

where $S_{t_{0}}(\tau)$ is the survival function or the probability that no event takes place in $\left(t_{0}, t_{0}+\tau\right]$, then:

$$
\frac{d S_{t_{0}}(\tau)}{d \tau}=\frac{d\left(1-F_{t_{0}}(\tau)\right)}{d \tau}=-f_{t_{0}}(\tau)
$$

and the relation (2.14) can be rewritten as:

$$
\mathbf{h}_{t_{0}}(\tau)=-\frac{d S_{t_{0}}(\tau)}{S_{t_{0}}(\tau) d \tau}=-\frac{d}{d \tau}\left(\ln S_{t_{0}}(\tau)\right)
$$

The term $\mathbf{h}_{t_{0}+u}(\tau) d \tau$ gives the probability of waiting time $\tau$ from $t_{0}+u$ onward, on condition that no event happens in $\left(t_{0}+u, t_{0}+u+\tau\right]$. If we also assume that no event has happened in $\left(t_{0}, t_{0}+u\right]$, then $S_{t_{0}+u}(\tau)=S_{t_{0}}(u+\tau) / S_{t_{0}}(u)$ and $\mathbf{h}_{t_{0}+u}(\tau)$ will be:

$$
\begin{aligned}
\mathbf{h}_{t_{0}+u}(\tau)= & -\frac{d}{d \tau}\left(\ln \frac{S_{t_{0}}(u+\tau)}{S_{t_{0}}(u)}\right)=-\frac{d}{d \tau}\left(\ln S_{t_{0}}(u+\tau)-\ln S_{t_{0}}(u)\right) \\
& =-\frac{d}{d \tau}\left(\ln S_{t_{0}}(u+\tau)\right) \\
& =\mathbf{h}_{t_{0}}(u+\tau) .
\end{aligned}
$$

This result helps simplifying our notations because $\mathbf{h}_{t_{0}}(\tau)$ is equal to $\mathbf{h}_{t_{0}+\tau}(0)$ as long as no event occurs in $\left(t_{0}, t_{0}+\tau\right]$.

It is important to mention that the concept of hazard function does not exclude the possibility of having simultaneous events. Indeed, standing at time $t$, the probability for the occurrence of at least one event on condition that no event occurs in $\left(t_{0}, t\right]$, is given by $\mathbf{h}_{t_{0}}\left(t-t_{0}\right)$ or $\mathbf{h}_{t}(0)$, where $\mathbf{h}_{t}(0) d t=P\left(\mathrm{~N}(t, t+d t] \geq 1 \mid S_{t}(0)\right)$. A point process $Z$ is called simple, if it does not include simultaneous events. This is called the simplicity property, and for $d t \rightarrow 0$ we will have:

$$
\mathbf{h}_{t}(0) d t \approx P\left(\mathrm{~N}(t, t+d t]=1 \mid S_{t}(0)\right)
$$

Below, I explain that the consideration of a simple point process implies that the hazard function at time $t$, and the time-dependent earthquake rate (or conditional intensity function) are approximately equal.

\section{Conditional intensity function}

The earthquake rate is time-dependent if $\lambda$ depends on the observation history $\mathcal{H}_{t}$ (what have happened up to time $t)$, thus the expected number of events $\mathrm{N}$, within $(t, t+\Delta t]$ will be:

$$
E\left(\mathrm{~N}(t, t+\Delta t] \mid \mathcal{H}_{t}\right)=P\left(\mathrm{~N}(t, t+\Delta t]=1 \mid \mathcal{H}_{t}\right)+\sum_{k=2}^{\infty} k P\left(\mathrm{~N}(t, t+\Delta t]=k \mid \mathcal{H}_{t}\right)=\lambda\left(t, \mid \mathcal{H}_{t}\right) \Delta t
$$


where, $\lambda\left(t \mid \mathcal{H}_{t}\right)$ is called conditional intensity function. For a point process $Z$ to be simple, no simultaneous events are assumed. Hence, very small $d t$ yields $P\left(\mathrm{~N}(t, t+d t]>1 \mid \mathcal{H}_{t}\right) \approx 0$ and:

$$
\lambda\left(t \mid \mathcal{H}_{t}\right) d t \approx P\left(\mathrm{~N}(t, t+d t]=1 \mid \mathcal{H}_{t}\right)
$$

which together with relation $(2.18)$ yield:

$$
\mathbf{h}_{t}(0) d t \approx \lambda\left(t \mid \mathcal{H}_{t}\right) d t
$$

Any suggested conditional intensity function for modelling the earthquake rate in time domain, involves the estimation of some unknown parameter values. The principal method for estimating these values with an optimal fit to our observations consists on maximizing the likelihood function of the proposed $\lambda\left(t \mid \mathcal{H}_{t}\right)$ (see Box 2.2).

\section{Stationary rate}

If the long-term average of earthquakes in unit of time or $\lambda$ is time-independent, then for any $\Delta t \subset\left[T_{i n i}, T_{\text {end }}\right]$ regardless of its location within $\left[T_{i n i}, T_{\text {end }}\right]$, the expected number of events is constant. This character yields the point process $Z$ to be stationary and for very small $\lambda \Delta t$ we have:

$$
\begin{aligned}
E(\mathrm{~N}(\Delta t))= & \lambda \Delta t=\lambda \Delta t e^{(-\lambda \Delta t)} e^{(\lambda \Delta t)}=\lambda \Delta t e^{(-\lambda \Delta t)} \sum_{k=0}^{\infty} \frac{(\lambda \Delta t)^{k}}{k !} \\
& =\lambda \Delta t e^{(-\lambda \Delta t)} \sum_{k=1}^{\infty} \frac{(\lambda \Delta t)^{k-1}}{(k-1) !}=e^{(-\lambda \Delta t)} \sum_{k=0}^{\infty} \frac{k(\lambda \Delta t)^{k}}{(k) !} \\
& =\sum_{k=0}^{\infty} k \frac{(\lambda \Delta t)^{k} e^{(-\lambda \Delta t)}}{(k) !} .
\end{aligned}
$$

Therefore we can write $P(\mathrm{~N}(\Delta t)=k)=\frac{(\lambda \Delta t)^{k} e^{(-\lambda \Delta t)}}{(k) !}$ that is the stationary Poisson distribution. Thus, the number of events in a very small time interval $\Delta t$ has a stationary Poisson distribution and $P(\mathrm{~N}(\Delta t)=1)=\lambda \Delta t e^{(-\lambda \Delta t)}$. Moreover, the equation (2.18) for a time-independent $\lambda$ gives:

$$
\mathbf{h}_{t_{0}}\left(t-t_{0}\right) \approx \lambda
$$

Using the relation (2.16), the survival function $S_{t_{0}}(\tau)$ can be rewritten as $\exp \left(-\int_{0}^{\tau} \mathbf{h}_{t_{0}}(u) d u\right)$, therefore we have:

$$
F_{t_{0}}\left(t-t_{0}\right)=1-\exp \left(-\int_{u=t_{0}}^{u=t} \lambda d u\right)=1-\exp \left(-\lambda\left(t-t_{0}\right)\right)
$$

Thus, the probability density function for the occurrence of one (equation (2.18) respects the simplicity property) event after time lag $\tau$ from $t_{0}$, only depends on $\tau$ :

$$
f_{t_{0}}(\tau)=\lambda \exp (-\lambda \tau)
$$




\section{Box 2.2}

\section{Likelihood function for the conditional intensity}

For an earthquake sequence with $N$ events occurring at times $\left\{t_{1}, t_{2}, \ldots, t_{n}\right\}$ inside a time interval $\left[T_{i n i}, T_{e n d}\right]$, if some events occur simultaneously then $n<N$. Otherwise, if there are no simultaneous events then $n=N$. Applying any stochastic point process model, the probability for the first event to take place at time $t_{1}$ is $f_{T_{\text {ini }}}\left(t_{1}-T_{\text {ini }}\right) d t$ and the probability for the $(i+1)$ th event to take place at time $t_{i+1}$ is $f_{t_{i}}\left(t_{i+1}-t_{i}\right) d t$, where $i=1,2, \cdots, n-1$. Thus the likelihood function is the joint probability for the happening of all events and not-happening of any event in $\left(t_{n}, T_{\text {end }}\right]$ :

$$
L\left(N ; T_{i n i}, T_{e n d}\right)=f_{T_{i n i}}\left(t_{1}-T_{i n i}\right) d t \times \prod_{i=1}^{i=n-1} f_{t_{i}}\left(t_{i+1}-t_{i}\right) d t \times S_{t_{n}}\left(T_{e n d}-t_{n}\right) .
$$

The survival function $S_{t_{0}}(\tau)$ can be rewritten as $\exp \left(-\int_{0}^{\tau} \mathbf{h}_{t_{0}}(u) d u\right)$ (see relation (2.16)), then the likelihood function $L$ will be:

$$
\begin{aligned}
L\left(N ; T_{\text {ini }}, T_{\text {end }}\right)= & \mathbf{h}_{T_{\text {ini }}}\left(t_{1}-T_{\text {ini }}\right) d t \times \exp \left(-\int_{T_{\text {ini }}}^{t_{1}} \mathbf{h}_{T_{\text {ini }}}\left(t-T_{i n i}\right) d t\right) \\
& \times \prod_{i=1}^{n-1}\left[\mathbf{h}_{t_{i}}\left(t_{i+1}-t_{i}\right) d t \times \exp \left(-\int_{t_{i}}^{t_{i+1}} \mathbf{h}_{t_{i}}\left(t-t_{i}\right) d t\right)\right] \\
& \times \exp \left(-\int_{t_{n}}^{T_{\text {end }}} \mathbf{h}_{t_{n}}\left(t-t_{n}\right) d t\right) \\
& =\mathbf{h}_{t_{1}}(0) d t \times \exp \left(-\int_{T_{\text {ini }}}^{t_{1}} \mathbf{h}_{t}(0) d t\right) \\
& \times \prod_{i=1}^{n-1}\left[\mathbf{h}_{t_{i+1}}(0) d t \times \exp \left(-\int_{t_{i}}^{t_{i+1}} \mathbf{h}_{t}(0) d t\right)\right] \\
& \times \exp \left(-\int_{t_{n}}^{T_{\text {end }}} \mathbf{h}_{t}(0) d t\right) \\
& =\prod_{i=1}^{n} \mathbf{h}_{t_{i}}(0) d t \times \exp \left(-\int_{T_{\text {ini }}}^{T_{\text {end }}} \mathbf{h}_{t}(0) d t\right) .
\end{aligned}
$$

Any suggested stochastic point process model that provides a higher value for the likelihood function in relation (B.27), gives a better solution for describing the earthquake process in the time domain. Sometimes, instead of $L$, the logarithm of the likelihood function or $L L$ is computed:

$$
\begin{aligned}
\ln L\left(N ; T_{\text {ini }}, T_{\text {end }}\right)= & \sum_{i=1}^{n} \ln \left(\mathbf{h}_{t_{i}}(0) d t\right)-\int_{T_{\text {ini }}}^{T_{\text {end }}} \mathbf{h}_{t}(0) d t \\
& =\sum_{i=1}^{n} \ln \mathbf{h}_{t_{i}}(0)-\int_{T_{\text {ini }}}^{T_{\text {end }}} \mathbf{h}_{t}(0) d t+C,
\end{aligned}
$$

where $\mathrm{C}$ is a constant parameter. For a point process with no simultaneous events where the conditional intensity at time $t$ depends on the observation history $\mathcal{H}_{t}$, the logarithm of 
likelihood function is:

$$
\ln L\left(N ; T_{\text {ini }}, T_{\text {end }}\right)=\sum_{i=1}^{n} \ln \lambda\left(t_{i} \mid \mathcal{H}_{t}\right)-\int_{T_{\text {ini }}}^{T_{\text {end }}} \lambda\left(t \mid \mathcal{H}_{t}\right) d t+C .
$$

\subsubsection{Omori-Utsu aftershock rate}

Following the seismic stress perturbation due to a mainshock magnitude $\mathbf{m}$ at time $t_{0}$, the aftershock frequency decays with time. This decay was first introduced by Omori (1894) as in equation (2.26), where $\tau$ is the elapsed time $\tau$ (or time lag), $\mathcal{K}_{\mathbf{m}}$ and $c$ are constants and $\nu_{\mathbf{m}}(\tau)$ is the aftershock rate (i.e. the number of aftershocks in unit of time) after a time lag $\tau$ since a parent mainshock with magnitude $\mathbf{m}$ :

$$
\nu_{\mathbf{m}}(\tau)=\mathcal{K}_{\mathbf{m}}(c+\tau)^{-1}
$$

Later, Utsu (1957) modified the Omori formula showing that the aftershock rate $\nu_{\mathbf{m}}$ at time $t$ agrees better with an inverse power law relation of the form:

$$
\nu_{\mathbf{m}}(t)=\mathcal{K}_{\mathbf{m}}\left(c+t-t_{0}\right)^{-p},
$$

where $p>0$ is the Omori power coefficient and $t_{0}$ is the mainshock occurrence time. Indeed, the aftershock rate at time $t$ is conditioned to the occurrence of a mainshock at time $t_{0}=t-\tau$. The equation (2.27) is also called Omori-Utsu formula. If we assume that all the seismicity at time $t$ is related to the aftershocks triggered by a single mainshock, then this relation represents the conditional intensity function:

$$
\lambda\left(t \mid \mathcal{H}_{t}\right)=\nu_{\mathbf{m}}(t)
$$

The value of $p$ indicates the rapidness of decay in the aftershock rate. A higher $p$ value points to a faster decay and a shorter aftershock duration. Ouillon \& Sornette (2005) observed a significant increase of $p$ with $\mathbf{m}$. The constant $\mathcal{K}_{\mathbf{m}} \propto e^{\alpha \mathbf{m}}$ represents the magnitude-dependent productivity and exponentially grows with $\mathbf{m}$, where the value of $\alpha>0$ varies from one study to the other and strongly depends on the aftershock selection algorithm (Hainzl \& Marsan, 2008).

Reasenberg \& Jones $(1989,1994)$ considered the probability distribution of aftershocks' magnitude, denoted by $f_{\mathbf{a}}(m)$, to forecast the aftershock activity due to a single mainshock using in the relation below:

$$
\nu_{\mathbf{m}}(t, m)=\frac{f_{\mathbf{a}}(m) \mathcal{K}_{\mathbf{m}}}{\left(c+t-t_{0}\right)^{p}}
$$

Another version of the Omori-Utsu formula that is introduced by Utsu (1971), is the multiple Omori-Utsu formula. This model says that not only the mainshock produces aftershocks, but also the large aftershocks can trigger their own aftershocks. Thus, the conditional intensity function 
is the summation of Omori-Utsu formula for the mainshock and for few $N_{l}$ largest aftershocks:

$$
\begin{aligned}
\lambda\left(t \mid \mathcal{H}_{t}\right)= & \nu_{\mathbf{m}}(t)+\sum_{i, t_{i}<t}^{N_{l}} \nu_{\mathbf{m}_{\mathbf{i}}}(t) \\
& =\mathcal{K}_{\mathbf{m}}\left(c+t-t_{0}\right)^{-p}+\sum_{i, t_{i}<t}^{N_{l}} \mathcal{K}_{\mathbf{m}_{i}}\left(c_{i}+t-t_{i}\right)^{-p_{i}}
\end{aligned}
$$

In this dissertation, I used the single Omori-Utsu formula (2.27) and its integration into the ETAS model (see section 2.1.4 and 2.1.7). The method of parameter estimation for this formula is explained in Box 2.3).

\section{Box 2.3}

\section{Omori-Utsu parameters estimation}

For estimating the Omori-Utsu parameters, the maximum likelihood method is commonly applied. The likelihood function for a conditional seismicity rate (aftershocks rate in this case) is defined in Box 2.2. For a mainshock of size $\mathbf{m}$ with total $N_{\mathbf{a}, \mathbf{m}}$ aftershocks occurring at times $\left\{t_{1}, t_{2}, \ldots, t_{N_{\mathbf{a}, \mathbf{m}}}\right\}$ inside a time interval $\left[t_{0}, t_{\mathbf{a}, \mathbf{m}}\right]$, by replacing the conditional intensity from single or multiple Omori-Utsu formula into the logarithm of likelihood function in equation (B.29), we will have:

$$
\ln L\left(N_{\mathbf{a}, \mathbf{m}} ; t_{0}, t_{\mathbf{a}, \mathbf{m}}\right)=\sum_{i=1}^{N_{\mathbf{a}, \mathbf{m}}} \ln \lambda\left(t_{i} \mid \mathcal{H}_{t}\right)-\int_{t_{0}}^{t_{\mathbf{a}, \mathbf{m}}} \lambda\left(t \mid \mathcal{H}_{t}\right) d t+C .
$$

However, Kagan \& Jackson (1995) discussed that right after a large mainshock, the small magnitude aftershocks become missed in recordings because their signals overlap with the mainshock coda and are unidentifiable. Thus, during a small time lag $\tau_{0}>0$, the observed number of aftershocks $\nu_{\mathbf{m}}\left(t \mid t<\left(t_{0}+\tau_{0}\right)\right)$, with magnitudes $m \geq m_{c}$, might be underestimated and this small period should be excluded in the process of estimating the Omori-Utsu parameters. Hence, assuming that all the earthquakes following a large mainshock are seismically triggered by that single parent, we can apply the Omori-Utsu formula to the aftershock sequence and estimate the value of $\mathcal{K}_{\mathbf{m}}, c$ and $p$ values by maximizing the relation above and using data since $t=t_{0}+\tau_{0}$. To do that, the openaccess software tool SAPP ${ }^{\mathrm{I}}$ by Ogata (2006), is used in this dissertation. This program minimizes the negative of $L L$ function above using the Davidon-Fletcher-Powell algorithm that begins with a set of initial values for the model parameters and repeats the calculation of $-L L$ values and its gradients at each step, until some convergence condition is met.

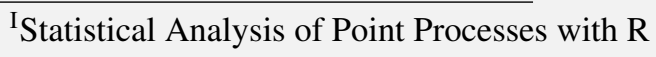




\subsubsection{Temporal ETAS modelling}

In chapter 1 (page 6) the expression background seismicity is explained as the earthquake population associated with the aseismic sources and not with the seismic triggering. In other words, this population comprises potential parents but not triggered children. Ogata (1988) generalized the multiple Omori-Utsu formula by not making distinctions between some few triggering events $\left(N_{l}\right.$ in relation (2.30)) and their aftershocks (triggered events). In turn, he proposed that each event, regardless its magnitude, can trigger its own offspring (Zhuang et al., 2012). This means that in addition to the background earthquakes, the aftershocks can also trigger other aftershocks and this branching process can continue until the end of studied time interval $T_{\text {end }}$. This model presents the earthquake rate as a combination of a constant background rate $\mu$, and a time-dependent aftershock rate $\nu$ :

$$
\lambda\left(t \mid \mathcal{H}_{t}\right)=\mu+\nu\left(t \mid \mathcal{H}_{t}\right)=\mu+\sum_{i, t_{i}<t} \nu_{\mathbf{m}_{i}}(t)
$$

and

$$
\nu_{\mathbf{m}_{i}}(t)=\frac{\mathcal{K}_{\mathbf{m}_{i}}}{\left(c+t-t_{i}\right)^{p}} .
$$

The term $\nu_{\mathbf{m}_{i}}(t)$ represents the aftershock rate at time $t$ and because of an event with magnitude $\mathbf{m}_{i}$ occurred at $t_{i}<t$ as in the Omori-Utsu formula. The summation is over all the events with magnitude $\mathbf{m} \geq m_{r}$ that have occurred inside the time interval $\left[T_{i n i}, t\right)$, where $m_{r}$ is called the reference magnitude and refers to the minimum magnitude for which the model is applied. This magnitude should not be lower than $m_{c}$, thus hereafter I use $m_{r}=m_{c}$. The productivity $\mathcal{K}_{\mathbf{m}_{i}}$, for each magnitude $\mathbf{m}_{i}$ is:

$$
\mathcal{K}_{\mathbf{m}_{i}}=\mathcal{K}^{*} e^{\alpha\left(\mathbf{m}_{i}-m_{c}\right)},
$$

where $\mathcal{K}^{*}$ is a constant value to be estimated. Ogata (1988) named the new formulations as Epidemic-Type Aftershock Sequence or ETAS model:

$$
\lambda\left(t \mid \mathcal{H}_{t}\right)=\mu+\sum_{i, t_{i}<t} \frac{\mathcal{K}_{\mathbf{m}_{i}}}{\left(c+t-t_{i}\right)^{p}} .
$$

The conditional intensity function in relation (2.34) for a marked point process incorporates the earthquake magnitude distribution. In the temporal ETAS model the earthquake magnitude, being from the background or from the aftershock population, is considered as an independent random variables with the same probability density distribution $f(m)$, for both populations, thus:

$$
\lambda\left(t, m \mid \mathcal{H}_{t}\right)=f(m)\left[\mu+\sum_{i, t_{i}<t} \frac{\mathcal{K}_{\mathbf{m}_{i}}}{\left(c+t-t_{i}\right)^{p}}\right] .
$$

\section{Branching ratio}

The ETAS model, as it is explained above, is a branching model, in which each event with any magnitude, triggers its own aftershocks and opens a new branch. Thus, for $T=\left[T_{\text {ini }}, T_{\text {end }}\right]$, the average number aftershocks directly triggered by an event of magnitude $\mathbf{m}_{i}$ occurred at $t_{i}$ is:

$$
E\left(\mathrm{~N}_{\mathbf{a}, \mathbf{m}_{i}}(T)\right)=\int_{t_{i}}^{T_{\text {end }}} \nu_{\mathbf{m}_{i}}(t) d t=A_{\Omega} \mathcal{K}^{*} e^{\alpha\left(\mathbf{m}_{i}-m_{c}\right)},
$$


where $A_{\Omega}=\frac{1}{C_{0}}-\frac{1}{(p-1)\left(c+T_{\text {end }}\right)^{p-1}}$ for $p \neq 1$, and $A_{\Omega}=\ln \left(T_{\text {end }}\right)-\ln (c)$ for $p=1$, and $C_{0}=$ $(p-1) c^{p-1}$. Therefore, the average number of direct aftershocks in the sequence is:

$$
E\left(\mathrm{~N}_{\mathbf{a}}(T)\right)=\int_{m_{c}}^{m_{\max }} E\left(\mathrm{~N}_{\mathbf{a}, \mathbf{m}_{i}}(T)\right) E(\mathrm{~N}(T)) f_{\mathbf{m}}(m) d m
$$

where $f_{\mathbf{m}}(m)$ is the probability density function for mainshock magnitude. Because any earthquake in the sequence can be a potential mainshock, then $f_{\mathbf{m}}(m)=f(m)$, where $m \in$ $\left[m_{c}, m_{\max }\right]$ is the earthquake magnitude, either background or aftershock. Therefore:

$$
\frac{E\left(\mathrm{~N}_{\mathbf{a}}(T)\right)}{E(\mathrm{~N}(T))}=\int_{m_{c}}^{m_{\max }} \int_{t_{m}}^{T_{\text {end }}} \nu_{m}(t) f(m) d t d m=A_{\Omega} \mathcal{K}^{*} \int_{m_{c}}^{m_{\max }} e^{\alpha\left(m-m_{c}\right)} f(m) d m .
$$

The ratio in relation (2.38) is called the "branching ratio", which is an index of direct triggering potential associated with the modelled sequence, and has a finite value between 0 and 1 . Its value shows the proportion of triggered events during the sequence (Helmstetter \& Sornette, 2002). Wherever the temporal ETAS model is applied in this dissertation, the branching ratio and its temporal variation by substituing $\Delta T=\left[T_{i n i}-t\right]$ with $T$ in the relation (2.38), are estimated and analysed. Although, I may not directly mention the derived values within the presented articles, they are taken into account whenever I conclude referring to the proportion of aftershocks contribution.

For the sake of further introduction to the spatiotemporal ETAS (section 2.1.7), I remind that another common representation of the temporal ETAS model is the relation (2.39), where $\mathcal{K}_{0}=\mathcal{K}^{*} A_{\Omega}$ and $\mathcal{T}\left(t-t_{i}\right)=1 / A_{\Omega}\left(c+t-t_{i}\right)^{p}$ is the probability density function for the aftershock time lag $t-t_{i}\left(T_{\text {end }} \ggg 1\right)$. If $p \leq 1$, the value of $A_{\Omega}$ does not converge, but for $p>1$ it does: $A_{\Omega} \rightarrow \frac{1}{C_{0}}$ and $\mathcal{T}\left(t-t_{i}\right) \rightarrow C_{0} /\left(c+t-t_{i}\right)^{p}$.

$$
\lambda\left(t \mid \mathcal{H}_{t}\right)=\mu+\sum_{i, t_{i}<t} \mathcal{K}_{0} e^{\alpha\left(\mathbf{m}_{i}-m_{c}\right)} \mathcal{T}\left(t-t_{i}\right)
$$

In this dissertation, a modified version of the temporal ETAS model that involves a timedependent background rate or $\mu(t)$ is used in chapters 3, 4 and 6. This version (Marsan et al., 2013) considers that the background rate is not actually constant and it might change with time. This model can characterize the temporal changes in the contribution of not only the aftershocks, but also the background earthquakes, and thus the temporal variation in the aseismic forcing. The applied methodology for the estimation of temporal ETAS parameters is explained in Box 2.4 .

\section{Box 2.4}

Temporal ETAS parameters estimation 
In order to estimate the five parameters in the temporal ETAS model $\left(\mu, \mathcal{K}^{*}, \alpha\right.$, $p$ and $c$ ) the maximum likelihood method should be applied to the conditional intensity function in relation (2.34). Applying the open-access software tool SAPP (Ogata, 2006) the value of $-L L$ is minimized with an iterative algorithm. However, in this dissertation, a different version of temporal ETAS model with time-dependent background rate (relation (B.40)) is used:

$$
\lambda\left(t \mid \mathcal{H}_{t}\right)=\mu(t)+\sum_{i, t_{i}<t} \frac{\mathcal{K}_{\mathbf{m}_{i}}}{\left(c+t-t_{i}\right)^{p}} .
$$

For estimating the temporal variation of background rate, together with value of four parameters $\mathcal{K}^{*}, \alpha, p$ and $c$, an algorithm developed by Marsan et al. (2013) and tested by Hainzl et al. (2013) is applied (via a software program in C, written and provided by Dr. S.Hainzl). This algorithm first assumes an initial constant value $\mu_{0}$ for the background seismicity and then it minimizes the negative of the log-likelihood function for the conditional intensity $\lambda\left(t \mid \mathcal{H}_{t}\right)$ (explained in Box 2.2) using the Davidon-FletcherPowell method and to estimate the parameters $\mathcal{K}^{*}, \alpha, p$ and $c$. Then, the achieved parameters are used to calculate a weight value $\omega_{i}$ (for $i=1,2, \ldots, N$ ) for each event at time $t_{i}$. This weight $\omega_{i}$ gives the probability of an event $i$ belonging to the background population and based on the achieved model so far:

$$
\omega_{i}=\frac{\mu_{0}}{\lambda\left(t_{i} \mid \mathcal{H}_{t}\right)} \text {. }
$$

Then, the time-dependent background rate is estimated choosing an smoothing window $n_{e}$ for selecting $n_{e}$ events prior and after event $i$. The time interval that covers the selected events will start at $t_{i-n_{e}}$ and will end at $t_{i+n_{e}}$. The background rate at time $t_{i}$ will be:

$$
\mu\left(t_{i}\right)=\frac{\sum_{j=i-n_{e}}^{i+n_{e}} \omega_{j}}{t_{i+n_{e}}-t_{i-n_{e}}} .
$$

Now, replacing the achieved $\mu\left(t_{i}\right)$ for all $t_{i}$ with constant $\mu_{0}$, the parameters are estimated again and this process continues iteratively until three convergence conditions are met. These conditions require adjusting of three factors until they become less than some relatively small and predefined limits. These factors are [1] the coefficient of variation ${ }^{\mathrm{I}}$ (or $\mathrm{CV}$ ) for a population of $\mu\left(t_{i}\right)$ in the actual iteration using a mean value that is estimated by the $\mu\left(t_{i}\right)$ values in the previous iteration, [2] the difference in Akaike information criterion $^{\mathrm{II}}$ (or $\delta \mathrm{AIC}$ ) between the actual and the previous iterations and [3] the difference in the total number of background events estimated by the model, related to the actual and the previous iterations.

The algorithm explained above is repeated for different smoothing windows and the best smoothing window is the one that gives the smallest $\mathrm{AIC}$ value, where $\mathrm{AIC}=2\left(\frac{N}{2 n_{e}+1}-L L\right)$ and $\frac{N}{2 n_{e}+1}$ is a proxy for the number of free parameters in $\mu(t)$ (Hainzl et al., 2013).

\footnotetext{
IThe CV shows the extent of variability in relation to the mean of the population and is defined as the ratio of the standard deviation to the mean.

II The AIC estimates the relative amount of information lost by a model and it is used to compare the quality of different models.
} 


\subsubsection{Seismicity density modelling}

In a stochastic point process modelling, we assume that the epicentre of an earthquake along time range $T$ is a random object in a two-dimensional finite space $\Phi$, and with the probability density $f(x, y)$, where the probability of earthquake occurrence inside $\left[x-\frac{d x}{2}, x+\frac{d x}{2}\right]$ and $\left[y-\frac{d y}{2}, y+\frac{d y}{2}\right]$ is $f(x, y) d x d y$. At any cell with centre at $(x, y) \in \Phi$ and size $d A=d x d y$, the observed earthquake density $\rho_{\text {obs }}(x, y)=n(x, y) / d A$, where $n(x, y)$ is the observed number of events inside $d A$. If we have $N$ events inside $\Phi$, then $P_{o b s}(x, y)$ or the observed probability of earthquake occurrence in $d A$, is $n(x, y) / N$ and thus $\rho_{o b s}(x, y)=N P_{o b s}(x, y) / d A$. If the spatial distribution of earthquakes has a spatial probability density function as $f(x, y)$, then the modelled earthquake density will be:

$$
\rho(x, y)=\frac{N P(x, y)}{d A}=N f(x, y)
$$

\section{Kernel function}

The shape of $f(x, y)$ can be derived using the epicentral coordinates of the earthquakes if a two-dimensional Kernel function $\mathbf{K}$ is applied to smooth the contribution of each epicentre $(E X, E Y)$ (e.g., Crespo Álvarez et al., 2013). A Kernel function is a probability density function and in the Cartesian system we have:

$$
\iint_{\mathbb{R}^{2}} \mathbf{K}(\mathrm{x}, \mathrm{y}) d \mathbf{x} d \mathbf{y}=1
$$

Applying this method, each earthquake with magnitude $m$ and epicentre at $(E X, E Y)$ is not a point any more, instead, it is associated with a bivariate probability density $\mathbf{K}(\mathrm{x}, \mathrm{y} \mid m)$ (Silverman, 1986), where $\mathrm{x}=x-E X$ and $\mathrm{y}=y-E Y$. The total density of earthquakes with magnitude $m$ at point $(x, y) \in \mathbb{R}^{2}$ derives from the summation on the Kernel values for all existing events with this magnitude whose epicentres are at $\left(E X_{i}, E Y_{i}\right)$, where $i=1,2, \ldots, n_{m}$ :

$$
\rho(x, y, m)=\sum_{i=1}^{n_{m}} \mathbf{K}\left(\mathrm{x}_{i}, \mathrm{y}_{i} \mid m\right),
$$

and for total $N$ events with $J$ magnitude classes $\mathbb{M}_{J}=\left\{m_{1}, m_{2}, \ldots, m_{J}\right\}$ within $\left[m_{0}, m_{\text {max }}\right]$ and number of repeats $\mathbb{N}_{J}=\left\{n_{m_{1}}, n_{m_{2}}, \ldots, n_{m_{J}}\right\}$, the total earthquake density at $(x, y)$ is:

$$
\rho(x, y)=\sum_{j=1}^{J} \rho\left(x, y, m_{j}\right) .
$$

Therefore the probability density function for the spatial distribution of epicentres $f(x, y)$, will be obtained by dividing $\rho(x, y)$ by $N$ :

$$
f(x, y)=\rho(x, y) / N
$$

Two of the most used Kernel functions with rotational symmetry for earthquake density computation are the Gaussian Kernel function:

$$
\mathbf{K}(\mathrm{x}, \mathrm{y} \mid m)=\frac{1}{2 \pi \mathrm{H}^{2}} \exp \left(-\frac{\mathrm{x}^{2}+\mathrm{y}^{2}}{2 \mathrm{H}^{2}}\right)
$$


and the inverse power law (IPL) Kernel function (equation (2.46)):

$$
\mathbf{K}(\mathrm{x}, \mathrm{y} \mid m)=\frac{q-1}{\pi \mathrm{H}^{2}}\left(1+\frac{\mathrm{x}^{2}+\mathrm{y}^{2}}{\mathrm{H}^{2}}\right)^{-q},
$$

where, $\mathrm{H}$ is the Kernel bandwidth, which is a magnitude-dependent parameter and $q>0$ is a Kernel parameter. For a Kernel function that replace a point data, the bandwidth $\mathrm{H}$ is a measure of the separation between points with the same size within a data set. The separation between the earthquakes with large magnitude is much bigger than the separation between the earthquakes with small magnitude. Figure 2.6 illustrates the shape of the Gaussian and the IPL Kernel distributions given in equations (2.45) and (2.46) in terms of epicentral distance $\sqrt{\mathrm{x}^{2}+\mathrm{y}^{2}}$.
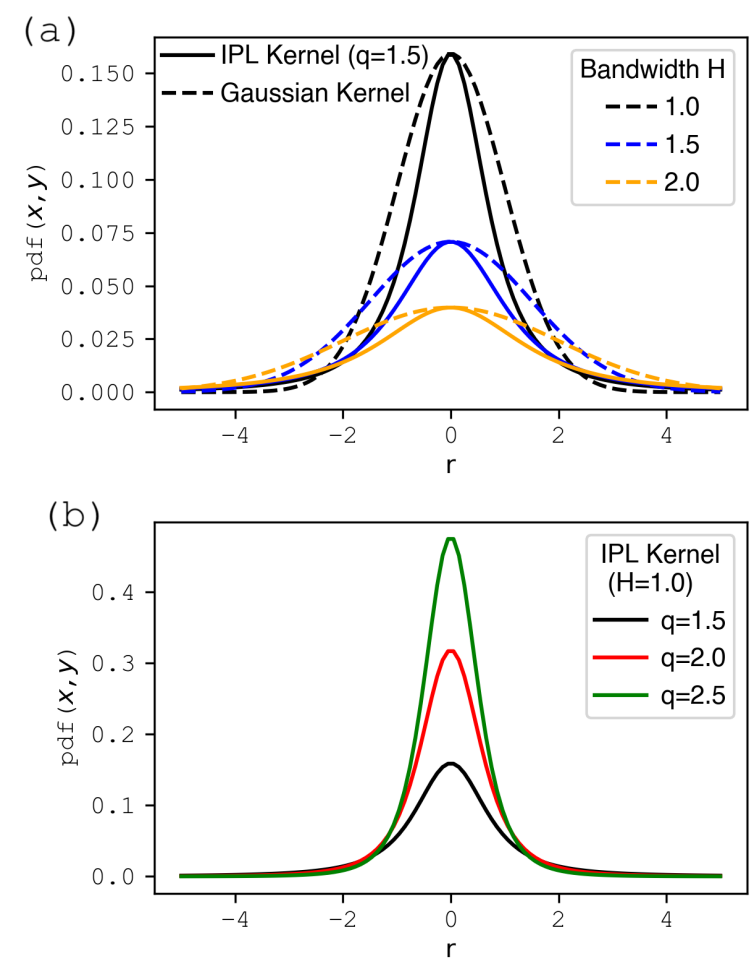

Figure 2.6: Comparison between the shapes of two most commonly used Kernel functions; Gaussian function in dashed lines and IPL function in solid lines. Influence of the bandwidth $\mathrm{H}$ in smoothing of both functions (a), and the influence of the $q$-value in the IPL function (b).

The bandwidth for smoothing an event with magnitude $m$ can be estimated by averaging the shortest distance-to-neighbour $\left(\mathrm{DTN}_{\min }\right.$ ) of the $n_{m}$ events with magnitude $m$ inside $\Phi$. Likewise, we can estimate $\mathrm{H}$ for all magnitude classes in $\mathbb{M}_{J}$ within a seismogenic zone. The result demonstrates an exponential relationship between the bandwidth and the magnitude:

$$
\mathrm{H} \propto e^{l m}
$$

where, $l$ is a parameter to be estimated. Figure 2.7 shows an example of this exponential relationship with $l=0.62$. For each magnitude class $m$, the $\operatorname{DTN}_{\min }$ is estimated for all $n_{m}$ events in that magnitude class. Then, the $\mathrm{DTN}_{m i n}$-values for each $m$ are modelled applying a normal distribution. The red curve shows the best fit that represents $\overline{\mathrm{DTN}}_{\text {min }}(m)$ or $\mathrm{H}(m)$. This relation is further applied for earthquake density estimations for different magnitude classes in chapter 6 . 


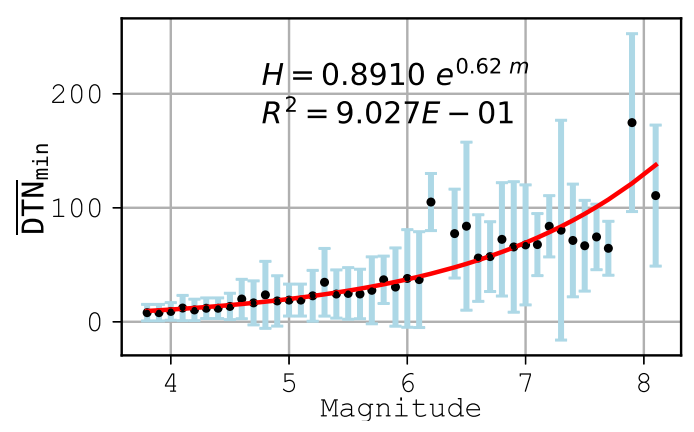

Figure 2.7: Black dots show the mean values $\overline{\mathrm{DTN}}_{\text {min }}$, and are accompanied by blue lines that represent the estimated $\pm \sigma$ for the normal distribution associate to each magnitude class $m$. The red line is the best exponential fit for $\overline{\operatorname{DTN}}_{\text {min }}(m)$. This figure is estimated applying the earthquakes in the catalog of National Seismological Service of Mexico along the southern coast with epicentre between longitudes $96^{\circ} \mathrm{W}$ and $105^{\circ} \mathrm{W}$ (see Figure 6 in chapter 6)

The choice for a bivariate Kernel function can have some type of asymmetry. It is likely that any asymmetry is related to some tectonic orientation which can easily be addressed by an azimuth angle $\Theta$. Indeed, using the polar coordinates is suitable for expressing the asymmetry of the Kernel function. Remembering that any probability density function $f$ in Cartesian coordinates will be transformed to a function $g$ in Polar coordinates as $g(r, \theta)=r f(x, y)$, where $r=\sqrt{x^{2}+y^{2}}$, and $\theta=\arctan \left(\frac{y}{x}\right)$ is the bearing angle 6 . If $\theta$ and $r$ are independent, then it can be written:

$$
\mathbf{K}(\mathbf{r}, \theta \mid m)=p d f(\mathbf{r} \mid m) p d f(\theta) \Longrightarrow p d f(\mathbf{r} \mid m) p d f(\theta)=\mathbf{r} \mathbf{K}(\mathrm{x}, \mathrm{y} \mid m)
$$

If the bivariate Kernel distribution is bearing-independent, then it has rotational symmetry and $p d f(\theta)$ is a constant value $\frac{1}{2 \pi}$. Thus, $\int_{0}^{2 \pi} p d f(\theta) d \theta=1$ and $p d f(\mathrm{r} \mid m)=2 \pi \mathrm{r} p d f(\mathrm{x}, \mathrm{y} \mid m)$. Equations (2.49) presents an example for a bearing-dependent probability density functions with reflectional symmetry. This Kernel function is also used by Woo (1996a).

$$
p d f(\theta)=\frac{1+\mathrm{w} \cos ^{2}(\theta-\Theta)}{\pi(2+\mathrm{w})},
$$

where $\Theta$ is the azimuth along which the spatial probability of the earthquake epicentre is aligned and $\mathrm{w}$ is the weighting parameter. Therefore, $p d f(\mathrm{r} \mid m)$ will be:

$$
p d f(r \mid m)=\frac{2 r(q-1)}{\mathrm{H}^{2}}\left(1+\frac{\mathrm{r}^{2}}{\mathrm{H}^{2}}\right)^{-q},
$$

and the product of equations (2.50) and (2.49) gives a bivariate Kernel distribution (equation (2.51)) that presents a spatial probability density as illustrated in Figure 2.8, for some orientations $\Theta$ and with $(E X, E Y)=(0,0)$. The figure also shows the impact of weighting parameter w. For $\mathrm{w}=0$, regardless of the introduced $\Theta$, the probability remains with radial symmetry. Higher values of $\mathrm{w}$ result in a more $\Theta$-aligned distribution, and $\mathrm{w}>1$ yields dumbbell shaped distributions with lower probabilities along $\Theta \pm \pi / 2$ orientations.

\footnotetext{
${ }^{6}$ The bearing angle of point $\mathrm{B}$ from point $\mathrm{A}$ is the azimuth of vector $\overrightarrow{A B}$
} 


$$
\begin{aligned}
\mathbf{K}(\mathrm{x}, \mathrm{y} \mid m)= & \frac{p d f(\mathrm{r} \mid m) p d f(\theta)}{\mathrm{r}} \\
& =\frac{2(q-1)}{\mathrm{H}^{2} \pi(2+\mathrm{w})}\left[1+\frac{\mathrm{w}}{2}\left(1+\cos 2 \Theta \frac{\mathrm{x}^{2}-\mathrm{y}^{2}}{\mathrm{x}^{2}+\mathrm{y}^{2}}+\sin 2 \Theta \frac{2 \mathrm{xy}}{\mathrm{x}^{2}+\mathrm{y}^{2}}\right)\right]\left(1+\frac{\mathrm{x}^{2}+\mathrm{y}^{2}}{\mathrm{H}^{2}}\right)^{-q} .
\end{aligned}
$$
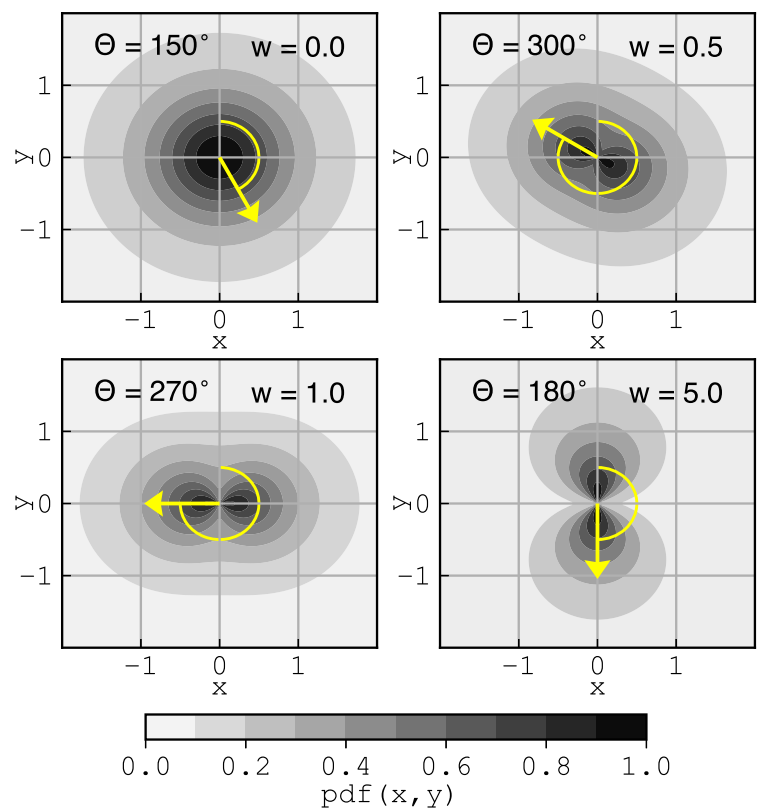

Figure 2.8: A comparison between the spatial probability density values in equation (2.51) for weighting parameters $0,0.5,1.0$ and 5.0 and different $\Theta$, with $\mathrm{H}=1$ and $q=1.5$.

In chapter 6 , the earthquake density rates $\dot{\rho}(x, y, m)$ for each magnitude $m \in[3.8,8.2]$ are computed using the equation (2.42) and the introduced Kernel function in the equation (2.51) (Figure 1.4). Figure 2.9 shows an example of smoothed density rate for $m=4.0$ and the participating events.

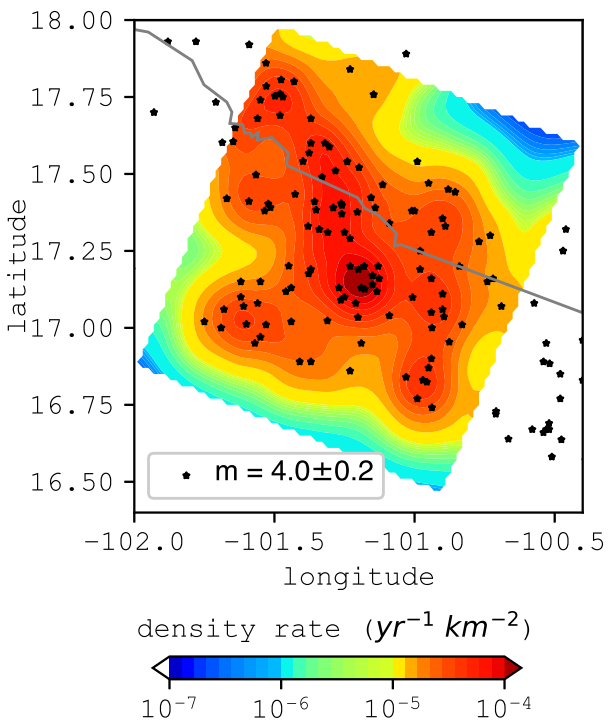

Figure 2.9: Surface projection of the annual density of subduction interface earthquakes for magnitude increments $m=4.0$ with $\pm 2 \sigma_{m}$ between 1992 and 2018 in the research zone of chapter 6 . 


\subsubsection{Aftershock density}

In previous sections the rate of aftershocks (of any magnitude) related to a mainshock of magnitude $\mathbf{m}$ was denoted by $\nu_{\mathbf{m}}(t)$. The term "rate" refers to the number of events in unit of time, whereas the term "density" refers to the number of events in unit of area. Here I denote the spatial density of aftershocks because of a mainshock with magnitude $\mathbf{m}$, with $\rho_{\mathbf{a}, \mathbf{m}}$, and thus, $\rho_{\mathbf{a}, \mathbf{m}}(t, x, y)$ is the spatial density of aftershocks (with any magnitude), at time $t$, and at $(x, y)$ with epicentral distance of $(\mathrm{x}, \mathrm{y})$ to the mainshock $\mathbf{m}$ at $\left(x_{0}, y_{0}\right)$. Standing at time $t \ggg 1$ long enough so all the $\mathrm{N}_{\mathrm{a}, \mathrm{m}}$ aftershocks have happened, we can write:

$$
\iint_{\Phi} \rho_{\mathbf{a}, \mathbf{m}}(t, x, y) d x d y=\mathrm{N}_{\mathbf{a}, \mathbf{m}}
$$

It is usually assumed that the aftershock epicentres are random and independent two-dimensional objects that are distributed with a probability density function $\mathcal{G}(\mathrm{x}, \mathrm{y} ; \mathbf{m})$ around the mainshock epicentre $\left(x_{0}, y_{0}\right)$, therefore at time $t \ggg 1$, and at any point $(x, y)$, where $\mathrm{x}=x-x_{0}$ and $\mathrm{y}=y-y_{0}$, their density is:

$$
\rho_{\mathbf{a}, \mathbf{m}}(t, x, y)=\mathrm{N}_{\mathbf{a}, \mathbf{m}} \mathcal{G}(\mathbf{x}, \mathrm{y} ; \mathbf{m}) .
$$

The aftershock spatial distribution $\mathcal{G}(\mathrm{x}, \mathrm{y} ; \mathbf{m})$ can be a bivariate Kernel function, with a smoothing parameter $\mathrm{H}_{\mathbf{a}, \mathbf{m}}$ that defines the spatial extent of aftershock area which is consistent with the area $\mathcal{A}$ ruptured by the mainshock. Experimental results by Kanamori (1970) indicate the consistency between the earthquake magnitude $\mathbf{m}$ and $\mathcal{A}$. Assuming that both the slip-parallel and slip-normal directions scale with the same characteristic length $\mathcal{L}$ (e.g., de Arcangelis et al., 2016) then $\mathrm{H}_{\mathbf{a}, \mathbf{m}} \sim \mathcal{L}$ and $\mathcal{A} \sim \mathcal{L}^{2}$. On the other hand, the results of empirical data (e.g., Wells $\&$ Coppersmith, 1994) for the seismic moment $\mathcal{M}$ show that:

$$
\mathcal{M} \propto \mathcal{A}^{3 / 2} \Rightarrow \mathcal{M} \propto \mathcal{L}^{3}
$$

The empirical relationships between seismic moment and earthquake magnitude also state (e.g., Nuttli, 1983; Stirling et al., 2013):

$$
\mathcal{M}_{\mathbf{m}} \propto 10^{\frac{3}{2} \mathbf{m}} \Rightarrow \mathcal{L} \propto 10^{0.5 \mathrm{~m}}
$$

and:

$$
\mathrm{H}_{\mathbf{a}, \mathbf{m}} \propto 10^{0.5 \mathbf{m}} .
$$

Some authors used the Gaussian Kernel function (equation (2.45)) for describing the distribution of epicentral distance of aftershocks (e.g., Zhuang et al., 2002; Console et al., 2003). Others used the inverse power law (IPL) function as in equation (2.46) (e.g., Ogata \& Zhuang, 2006; Zhuang et al., 2002, 2005). The bandwidth $\mathrm{H}_{\mathbf{m}}$, as it was also discussed above has an exponential relationship with mainshock magnitude $\mathbf{m}$ :

$$
\mathrm{H}_{\mathbf{a}, \mathbf{m}} \propto e^{\gamma \mathbf{m}} .
$$

In the following section, the temporal ETAS model (see section 2.1.4) is modified to also consider the spatial distribution of earthquakes. Thus, the new term $\nu_{\mathbf{m}}(t, x, y)$ will be incorporated in the formulation representing the "density rate" of aftershocks due to a mainshock of magnitude $\mathbf{m}$. In fact $\nu_{\mathbf{m}}(t, x, y)$ is another notation for $\dot{\rho}_{\mathbf{a}, \mathbf{m}}(t, x, y)$. 


\subsubsection{Spatiotemporal ETAS modelling}

Before Ogata generalized the temporal ETAS model (relation (2.34)) to the spatiotemporal version, other authors used space-time clustering models with slightly different forms (e.g, Kagan, 1991; Musmeci \& Vere-Jones, 1992; Rathbun, 1993). The conditional intensity of the spatiotemporal ETAS model defined by Ogata (1998) is given by:

$$
\lambda\left(t, x, y \mid \mathcal{H}_{t}\right)=\mu(x, y)+\sum_{i, t_{i}<t} \nu_{\mathbf{m}_{i}}(t, x, y)
$$

with spatiotemporal formulation of aftershock rate as:

$$
\nu_{\mathbf{m}_{i}}(t, x, y)=\mathcal{K}_{0} e^{\alpha\left(\mathbf{m}_{i}-m_{c}\right)} \mathcal{T}\left(t-t_{i}\right) \mathcal{G}\left(x-x_{i}, y-y_{i} ; \mathbf{m}_{i}\right)
$$

where $\mathcal{G}\left(x-x_{i}, y-y_{i} ; m_{i}\right)$ is the bi-variate probability density function that describes the spatial distribution of aftershocks due to event $i$ with magnitude $\mathbf{m}_{i}$. Some authors choose a bivariate Gaussian Kernel function for the aftershock distribution as in equation (2.45) (e.g., Ogata, 1998; Zhuang et al., 2002; Console et al., 2003). Some other authors use IPL Kernel function as in equation (2.46) (e.g., Ogata, 1998; Zhuang et al., 2005; Ogata \& Zhuang, 2006). In this dissertation the spatiotemporal ETAS modelling is applied in chapter 6 and for parameters estimation, the $\mathrm{SEDA}^{7}$ software by Lombardi (2017) is used. This software considers a symmetric IPL Kernel function as introduced by Zhuang et al. (2005) and Ogata \& Zhuang (2006):

$$
\begin{gathered}
\mathcal{G}\left(x-x_{i}, y-y_{i} ; \mathbf{m}_{i}\right)=\frac{q-1}{\pi \mathrm{H}_{\mathbf{a}, \mathbf{m}_{i}}^{2}}\left(1+\frac{\left(x-x_{i}\right)^{2}+\left(y-y_{i}\right)^{2}}{\mathrm{H}_{\mathbf{a}, \mathbf{m}_{i}}^{2}}\right)^{-q} \\
\lambda\left(t, x, y \mid \mathcal{H}_{t}\right)=\mu(x, y)+\sum_{i, t_{i}<t} \frac{\mathcal{K}_{0} e^{\alpha\left(\mathbf{m}_{i}-m_{c}\right)}}{A_{\Omega}\left(c+t-t_{i}\right)^{p}} \frac{A_{\Phi}}{\left(\mathrm{H}_{\mathbf{a}, \mathbf{m}_{i}}^{2}+r_{i}^{2}\right)^{q}}
\end{gathered}
$$

where $A_{\Phi}=\left[(q-1)\left(\mathrm{H}_{\mathbf{a}, \mathbf{m}_{i}}^{2}\right)^{q-1}\right] / \pi$ is a normalization constant and $\mathrm{H}_{\mathbf{a}, \mathbf{m}_{i}}=d e^{\gamma\left(\mathbf{m}_{i}-m_{c}\right)}$. Indeed, for modelling the earthquake sequence in time and space using the spatiotemporal ETAS in relation (2.61), the eight parameters $\mathcal{K}^{* *}, \alpha, p, c, q, d$ and $\gamma$ have to be estimated, where $\mathcal{K}^{* *}=\mathcal{K}_{0} A_{\Phi} / A_{\Omega}$. The normalization constant $A_{\Omega}$ was previously defined in section 2.1.4.

Same as in the marked point process for the temporal ETAS modelling, the size (i.e. magnitude) of all the events, including background and aftershocks is considered as an independent random value drawn from the same probability density function $f(m)$. Thus, the conditional intensity can be composed as:

$$
\lambda\left(t, x, y, m \mid \mathcal{H}_{t}\right)=f(m) \lambda\left(t, x, y \mid \mathcal{H}_{t}\right) .
$$

\footnotetext{
${ }^{7}$ Statistical earthquake data analysis
} 


\subsection{Earthquake re-location}

An earthquake sequence involves several earthquakes that occur within a certain spatiotemporal window. By being grouped, it is supposed that they share same origin and common source/s of occurrence. When we apply statistical modelling, we consider the earthquakes with exact origin time and exact location. These data come from earthquake catalogs, which accuracy determines the reliability of any statistical analysis.

Earthquake locating process includes the determination of both hypocentral coordinates and origin time of first energy release through different techniques. The location may refer to the earthquake's hypocenter or centroid (see page 42). A commonly applied method for finding the hypocenter of a single earthquake, basically uses the difference between the arrival time of seismic phases $\mathrm{P}$ and $\mathrm{S}$ at a seismic station to calculate the distance of earthquake source to the station via travel-time tables of seismic phases (e.g, IASPE91 table in Figure 2.10, ak135, PREM). For IASPE91, Kennet (1991) used millions of seismic phases arrival times recorded at thousands of stations all over the world during more than two decades and constructed IASPE91 tables for variety of source depths. A minimum of three seismograms recorded on three different stations is necessary for triangulation of the epicentral distance. Typically, the uncertainty is many times larger than the source dimension of the event itself. A large number of involved stations and a good azimuthal coverage helps reducing the level of uncertainties. However, this method is the preferred one and it is applied for many earthquake catalogs because it is simple and straightforward.

The determination of the earthquake depths is usually more complicated. For local earthquakes, the source is generally beneath the stations and they mostly detect the up-going waves. On the contrary, for regional earthquakes over the planet the stations receive down-going waves. For determining the earthquake depths, the arrival times of seismic phases that are reflected from the Earth surface are more important, thus published travel-time tables for different depth are usually applied (Spence et al., 1989).

Some other techniques can determine the relative location, where the location of an earthquake becomes computed relative to a master event (e.g., Deichmann \& Garcia-Fernandez, 1992) or relative to each other as in double-difference or DD method. This method is used in this dissertation Waldhauser \& Ellsworth (2000).

The double-difference method is called as a re-location method because it first uses an absolute hypocentre coming from earthquake catalogs. The accuracy of re-locating approach depends on the accuracy of each event locating and also the applied seismic velocity model. These models provide $V_{P}$ and $V_{S}$ (velocity of seismic phases $P$ and $S$ respectively), within different layers or the Earth and come from inverted travel-times or seismic tomography (e.g, Shearer, 2019). The uncertainties by double-difference method can be the result of random errors in the raw observations or systematic biases, which are not reflected in the formal uncertainties provided with the catalog (Husen \& Hardebeck, 2010). This method first searches for pairs of events (event $i$ and event $j$ ) that have the same registered seismic phase/s in the same stations $k$. Then it 


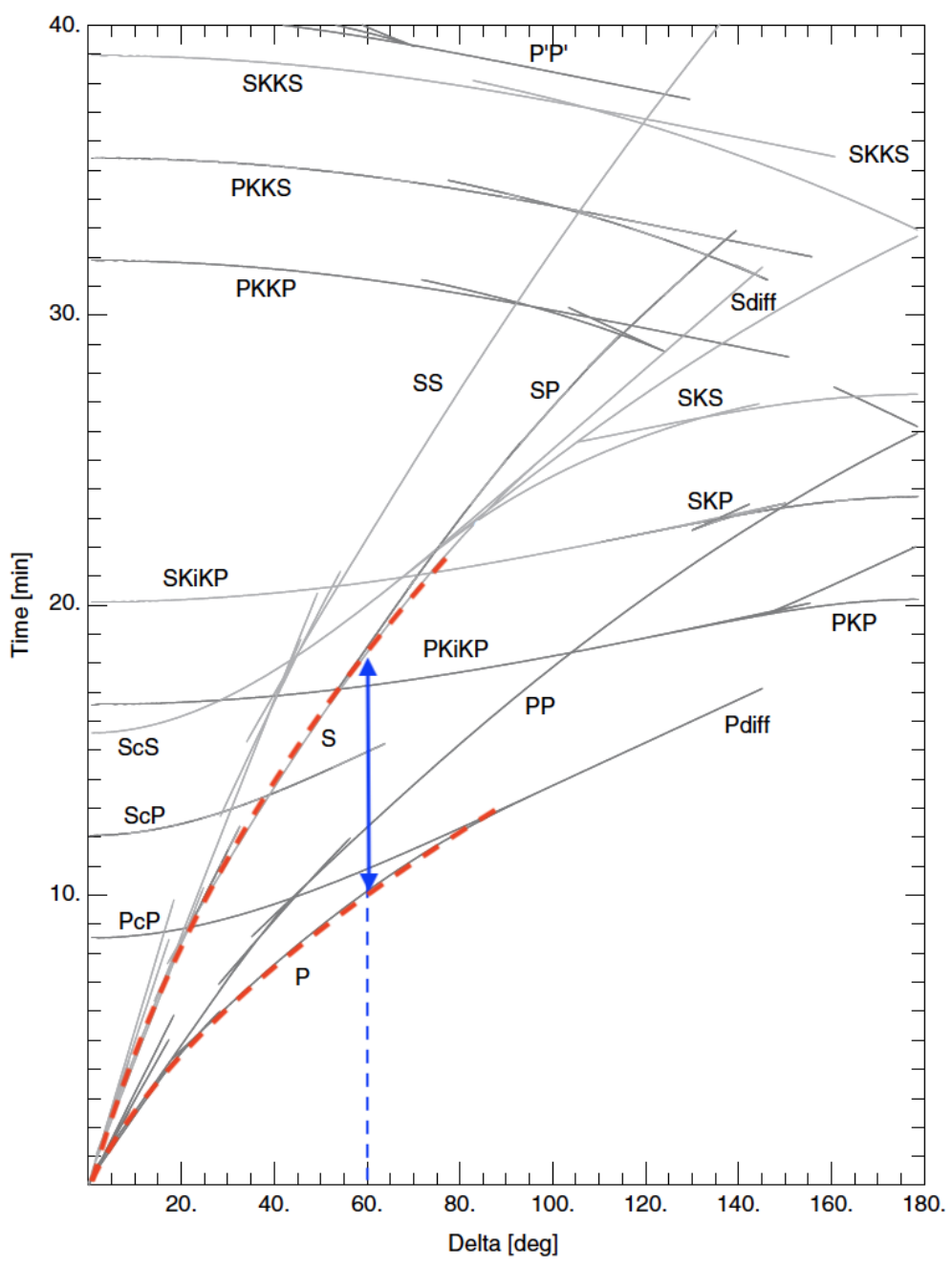

Figure 2.10: Graph of travel times of seismic phases identified in the IASPE 1991 Seismological Tables. This figure is fromStacey et al. (1977), pp 274, and the coloured features are added later. The red dashed curves highlight the $\mathrm{P}$ and $\mathrm{S}$ travel times. The two-sides arrow shows the difference between arrival time of seismic phases $\mathrm{P}$ and $\mathrm{S}$ for radial distance of 60 degrees.

constructs the $D D$ equation for that common phase $\Phi$ :

$$
D D_{k, \Phi}^{i, j}=\left(t_{k, \Phi}^{i}-t_{k, \Phi}^{j}\right)^{o b s}-\left(t_{k, \Phi}^{i}-t_{k, \Phi}^{j}\right)^{c a l c} .
$$

Where $t_{k, \Phi}^{i}$ is the difference between the exact time of earthquake $i$ (the time given in the catalog) and the arrival time of its phase $\Phi$ at station $k$. Assuming that the hypocentral separation between two earthquakes is small compared to the event-station distance, the travel path for both would be very similar (Got et al., 1994). The best situation is when these paths are nearly identical, because the difference $\left(t_{k, \Phi}^{i}-t_{k, \Phi}^{j}\right)$ would be due to the adjustment in the relative hypocentre and time of the earthquake $i$ and $j$. Therefore, $\left(t_{k, \Phi}^{i}-t_{k, \Phi}^{j}\right)^{\text {calc }}$ in each $D D$ equation, includes information about hypocentre and time (4 parameters) of two earthquakes, and the degrees of freedom are eight. Hence, if there are eight observations of common phase in common stations for each pair, then these equations can be solved without fixing any unknown parameters.

However, minimizing many $D D$ equation allows simultaneous relocation of large number of earthquakes and it works best if events are densely distributed (compared to the average station 
spacing) and observed at a large number of stations (Waldhauser \& Ellsworth, 2000; Husen \& Hardebeck, 2010).

\subsection{Static stress modelling}

An earthquake happens when the frictional state over a fault plane becomes unstable and the plane starts rupturing. The time span separating two consecutive ruptures is called interseismic period. Actually, it can be periodic or not (Figure 2.12). During the interseismic period, the locking of the plate interface produces accumulation of strain due to an ongoing tectonic process (Scholz, 2019). For this reason, the interseismic period is also called the loading cycle. The instability and rupture growth depend on the initial conditions over the fault plane. One of the most widely used criteria for characterizing the physical condition under which failure occurs in a rock, is the Coulomb failure criterion. This criterion associates rock failure with the exceedance of a specific value of an accumulating static stress (King et al., 1994; Stein, 1999). For each assumed fault plane with an uniform static coefficient of friction $\mu_{f}$, the Coulomb failure stress is defined as:

$$
\mathrm{CFS}=\left|\sigma_{s}\right|+\mu_{f}\left(\sigma_{n}+\mathrm{p}_{\text {pore }}\right),
$$

where $\left|\sigma_{s}\right|$ is the absolute value of the tectonic shear stress $\overrightarrow{\sigma_{s}}$, over the fault plane, $\sigma_{n}$ is the amount of normal stress $\overrightarrow{\sigma_{n}}$ to the fault plane (positive for extension) and $\mathrm{p}_{\text {pore }}$ is the fluid pressure in the rock pores (negative in compression, favouring extension relative to the fault). Failure occurs in the direction of shear stress $\overrightarrow{\sigma_{s}}$ when CFS overcomes an internal strength or cohesion $\mathbf{S}_{\mathbf{0}}$. This would happen when the amount of shear stress $\left|\sigma_{s}\right|$ to the fault increases, or the resistance of fault against rupture reduces. Reduction of fault's resistance can be due to a decrease in the compressional normal stress $\sigma_{n}$, or an increase in the pore pressure pore, or both. (Harris, 2000; de Arcangelis et al., 2016). The term $\sigma_{n}+\mathrm{p}_{\text {pore }}$ is also referred as the effective normal stress. Using equation (2.64) to model ruptures over huge blocks of overburden rocks is challenging as it requires data on resultant stress fields and physical properties of rocks as porosity, pore pressure and friction.

\section{Box 2.5}

\section{Stress tensor}

Any surface $\overrightarrow{\mathbf{A}}$, in Cartesian notation with normal unit vectors $\hat{\mathbf{x}}_{1}, \hat{\mathbf{x}}_{2}$ and $\hat{\mathbf{x}}_{3}$ is defined by:

I) its normal vector $\hat{\mathbf{n}}=n_{1} \hat{\mathbf{x}}_{1}+n_{2} \hat{\mathbf{x}}_{2}+n_{3} \hat{\mathbf{x}}_{3}$ of unit size, where $n_{1}^{2}+n_{2}^{2}+n_{3}^{2}=1$, and II) its area $\mathbf{A}$.

Therefore, $\overrightarrow{\mathbf{A}}=\mathbf{A} \cdot \hat{\mathbf{n}}$, where $\hat{\mathbf{n}}$ presents a unit of area on surface $\overrightarrow{\mathbf{A}}$. For such unit area under stress, the applied force because of the material in the positive side acting on the material in the negative side can be computed using the stress tensor $\mathrm{T}$. The stress tensor in three dimensions is a table of nine coefficients $\left[\sigma_{i j}\right]$, where $\sigma_{i j}$ presents the force acting in direction $i$ on an unit area of a plane with normal $j$ (Figure 2.11). In Geophysics, the most commonly used unit for describing the amount of stress is "bar". One bar is equal to 
$10^{5}$ Pascal $\left(\mathrm{N} . \mathrm{m}^{-2}\right)$ or $10^{6}$ dyn. $\mathrm{cm}^{-2}$. Assuming that the stress tensor in the Cartesian coordinates is:

$$
\mathbf{T}=\left[\begin{array}{lll}
\sigma_{11} & \sigma_{12} & \sigma_{13} \\
\sigma_{21} & \sigma_{22} & \sigma_{23} \\
\sigma_{31} & \sigma_{32} & \sigma_{33}
\end{array}\right]
$$

then the force applied to unit area $\hat{\mathbf{n}}$ can be derived by:

$$
\mathbf{T} \cdot \hat{\mathbf{n}}=\left[\begin{array}{lll}
\sigma_{11} & \sigma_{12} & \sigma_{13} \\
\sigma_{21} & \sigma_{22} & \sigma_{23} \\
\sigma_{31} & \sigma_{32} & \sigma_{33}
\end{array}\right]\left[\begin{array}{l}
n_{1} \\
n_{2} \\
n_{3}
\end{array}\right]=\left[\begin{array}{c}
\mathrm{T}_{1 \hat{\mathbf{n}}} \\
\mathbf{T}_{2 \hat{\mathbf{n}}} \\
\mathbf{T}_{3 \hat{\mathbf{n}}}
\end{array}\right]=\overrightarrow{\mathbf{T}}_{\hat{\mathbf{n}}},
$$

where $\mathbf{T}_{i \hat{\mathbf{n}}}=\sum_{j=1}^{3} \sigma_{i j} n_{j}$ is the force along $\hat{\mathbf{x}}_{i}$ direction acting on unit area of surface $\overrightarrow{\mathbf{A}}$. Thus, the force along $\hat{\mathbf{x}}_{i}$ acting on whole surface $\overrightarrow{\mathbf{A}}$ is: $\mathbf{A} T_{i \hat{\mathbf{n}}}$. The force $\overrightarrow{\mathbf{T}}_{\hat{\mathbf{n}}}=$

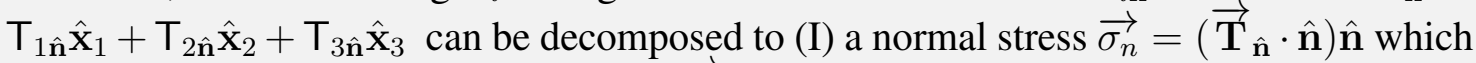
acts perpendicular to area unit of surface $\overrightarrow{\mathbf{A}}$ (In this dissertation the positive normal stress presents the extension), and (II) a shear stress $\vec{\sigma}_{s}=\overrightarrow{\mathbf{T}}_{\hat{\mathbf{n}}}-\vec{\sigma}_{n}$ which acts tangent to area unit of surface $\overrightarrow{\mathrm{A}}$ (Figure 2.11), thus:

$$
\overrightarrow{\mathbf{T}}_{\hat{\mathbf{n}}}=\vec{\sigma}_{n}+\vec{\sigma}_{s}
$$
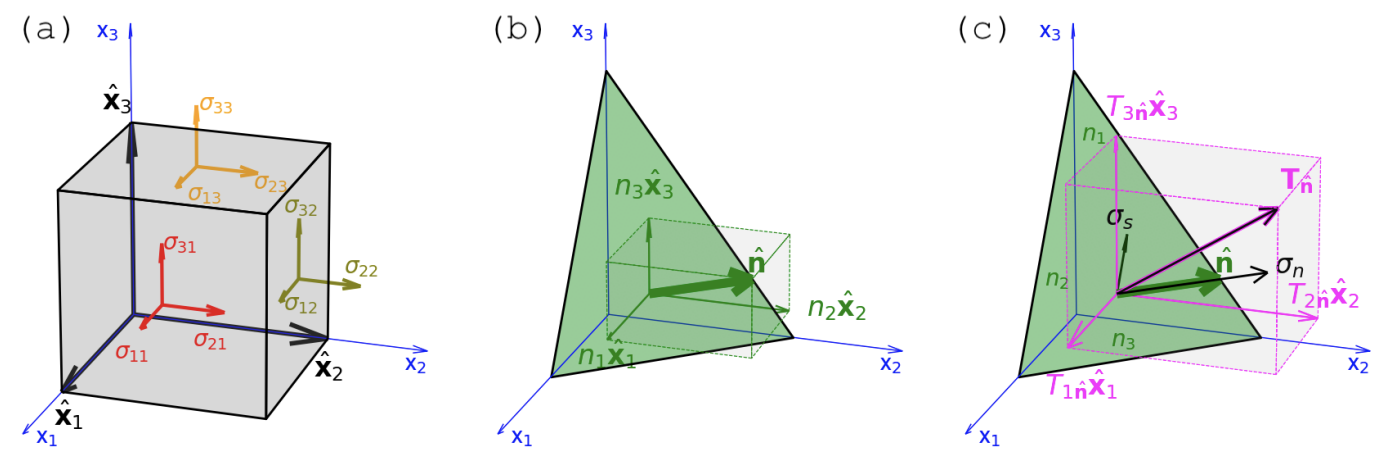

Figure 2.11: The stress component $\sigma_{i j}$ showing the force acting in the direction of $i$ on an unit area of a plane with normal $j$ for positive side of volume increment (a). An unit of area with normal $\hat{\mathbf{n}}$ and its three orthogonal components of area (b). A force $\overrightarrow{\mathbf{T}}_{\hat{\mathbf{n}}}$ and its three orthogonal components acting on the unit area $\hat{\mathbf{n}}$ (c). The vectors $\sigma_{n}$ and $\sigma_{s}$ are the normal and the tangential components respectively.

The Geodetic measurements and derived slip velocity data can help us to model the elastic dislocation from crustal movements and to estimate the orientations of regional principal stresses in the region, but it is not possible to measure directly the regional amount of stress and to determine the CFS over a fault plane.

Figure 2.12 shows different models of seismic cycles described by Shimazaki \& Nakata (1980). These cycles can be used to quantify stress changes with respect to the last rupture. However, this 
approach for studying interseismic cycles of fault failures is very limited. For large earthquakes, these periods are generally about hundred years or more, while instrumental seismic and geodetic data are available since less than a century in most parts of the World. On the other hand, the interseismic cycle involves other smaller earthquakes and other stress-controlling mechanisms as pore pressure diffusion, slow slip events, tectonic deformation, etc. which modify the rate of stress accumulation. Such mechanisms entail a stronger alteration of the cycle for smaller earthquakes. Overall, it is not feasible to estimate the actual amount stress over a determined fault plane and forecast when its locked interface is going to slide.

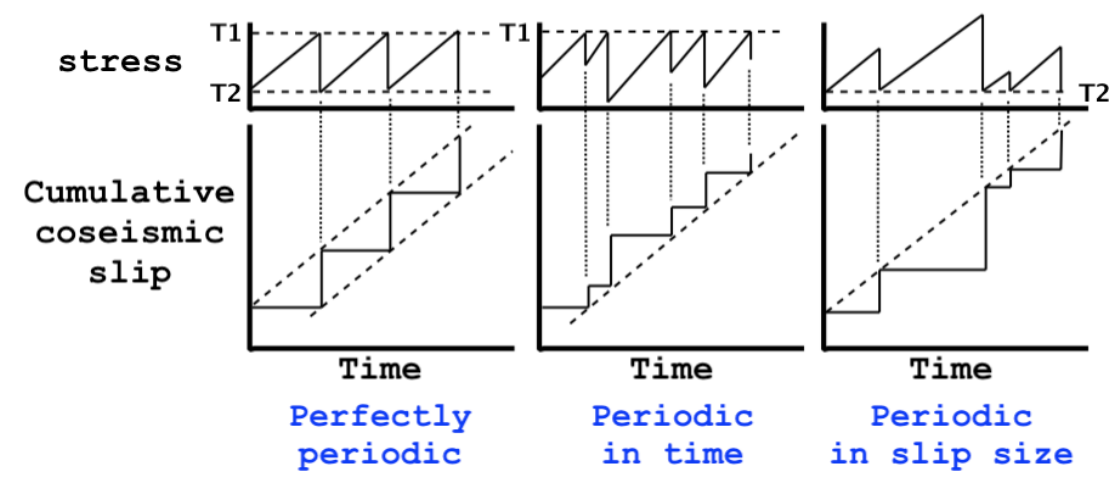

Figure 2.12: Simple recurrence models by Shimazaki \& Nakata (1980).

Nevertheless, the occurrence of any deformation in the elastic medium generates computable CFS changes over nearby fault planes or any other potential rupture area. For instance, when an earthquake, say EQ1 happens, the overburden rock breaks along a fault and the rupture propagates over the fault's surface. As a result, the two sides of the fault plane slide with respect to each other and the material on both sides of the ruptured plane is displaced and deformed. This deformation after the occurrence of EQ1 changes the actual stress tensor T (see Box 2.5) that affects the material. To calculate this change it is necessary to first model the produced deformation.

The displacements and deformations around rupture plane located in a half-space (along the earth's surface and below it) are described by a vector field $U$ and its partial derivatives. The earthquake displacement field $\mathbf{U}$, can be modelled only if we have enough information about the geometry and location of the seismic source, namely the amount of slip along the rupture plane and the elastic properties of the material where the rupture takes place. Converse (1973) derived $\mathbf{U}(x, y, z)$ and its partial derivatives for any point $(x, y, z)$ in the elastic half-space for any given elastic moduli. Yang \& Davis (1986) formulated U considering opening motion across the rupturing surface too (Erickson, 1987). Okada (1992) also developed the dislocation formula to calculate $\mathbf{U}(x, y, z)$ in an elastic half-space with uniform, isotropic elastic properties. All these approaches show that a comprehensive description of the earthquake location, source dimensions and geometry plus its slip model are crucial.

In this section, first the slip modelling for an earthquake rupture is introduced. It is explained how the dislocation field $\mathrm{U}$ associated to an earthquake $E Q 1$, produces changes in the CFS amount 
on any hypothetical planes nearby. Finally, the physical concepts and formulations related to the estimations of Coulomb failure stress change ( $\triangle \mathrm{CFS}$ ) are described.

\subsubsection{Earthquake source modelling}

An earthquake involves a sudden slip with average amount $\overline{\mathrm{s}}$, over a ruptured area of size $\mathcal{A}$, and a seismic moment release of $\mathcal{M}_{0}=\mathrm{G} \mathcal{A} \overline{\mathrm{s}}$, where $\mathrm{G}$ is called the shear modulus (Aki, 1966). This slip starts at a specific location called hypocentre and in an specific direction given by the rake angle or $\boldsymbol{\lambda}$. The rake direction (assumed to be consistent over the entire rupture area) together with the fault plane orientation describe the focal mechanism of the earthquake. In order to determine the earthquake hypocentre and focal mechanism, it is necessary to use a significant number of high quality seismic records. From these records, we can extract data on the arrival time and duration of different types of seismic waves and their phases. Applying inversion methods we can translate these data into information about the source of rupture.

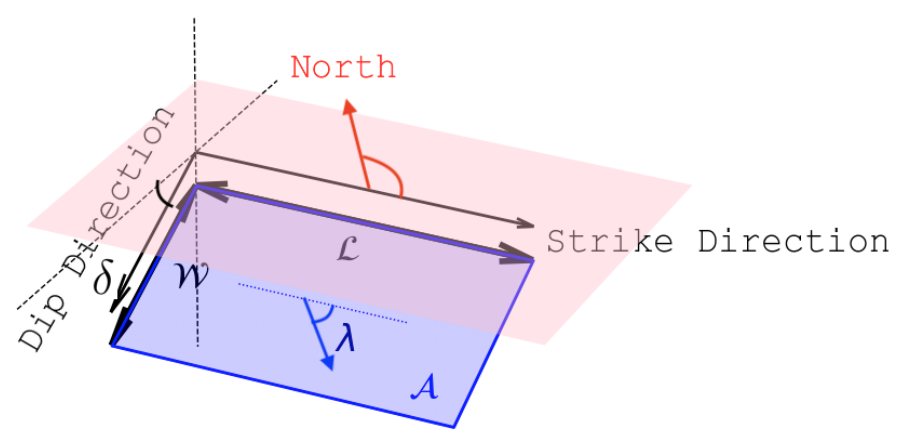

Figure 2.13: Schematic view of a simple a rectangular fault. The strike angle is the angle that the surface projection of the fault's upper edge makes with the north direction $\left(0^{\circ} \leq\right.$ strike angle $\left.<360^{\circ}\right)$ and in a way that the dip direction stays on its right hand side. The dip angle is measured from the surface. The rake angle is the slip angle on the fault plane, where $\boldsymbol{\lambda}=0^{\circ}$ indicates a pure left-lateral slip, $\boldsymbol{\lambda}=180^{\circ}$ a pure right-lateral slip, $0^{\circ}<\boldsymbol{\lambda}<180^{\circ}$ shows upward slip and $-180^{\circ}<\boldsymbol{\lambda}<0^{\circ}$ downward slip.

Seismic wave inversion methods are also used for modelling the slip distribution over each rupture patch $\delta \mathcal{A}$. In reality, the slip amount is not distributed homogeneously over $\mathcal{A}$. For instance, if the shear modulus is constant along the fault, the region of mean seismic moment release or centroid ${ }^{8}$ is usually near the maximum slip. Moreover, the location of maximum slip can coincide with the location of the hypocentre or not. The uncertainties in the slip model lead to serious uncertainties in the $\Delta$ CFS estimation (King \& Cocco, 2001). One of the fundamental methods for determining the earthquake slip model is Finite-Fault source inversion. These inversions use seismic ground-motion recordings to quantify the spatial distribution and direction of slip on the fault. The resolution and reliability of each model depend mainly on the quantity and quality of strong-motion data (Ide, 2007) and $\triangle$ CFS maps are highly influenced by the obtained slip models. Finite fault inversion that applies seismic, high-rate GPS or tsunami data, can also model the kinematics of rupture and the spatiotemporal evolution of coseismic

\footnotetext{
${ }^{8}$ Located at the barycentre of the seismic slip distribution, both in space and time, represents a point source with equivalent mechanism.
} 
displacement (e.g., how fast the rupture expanded over the fault surface, and how long each point on the fault slipped) (Ji et al., 2002; Martin Mai et al., 2016).
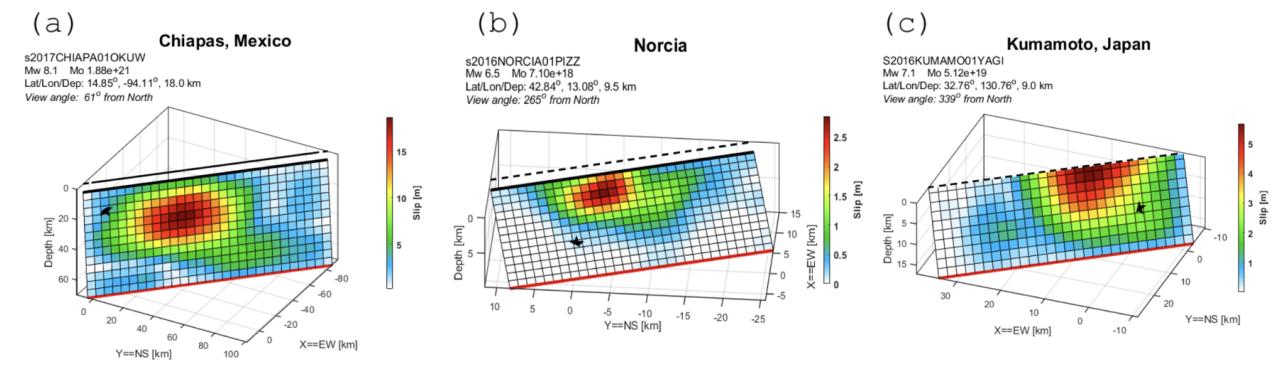

Figure 2.14: Examples of slip model solved for previous earthquakes worldwide, and downloaded from Finite-Fault Earthquake Source Model Database (Mai \& Thingbaijam, 2014).

However, most of the time, it is not feasible to find or compute a reliable slip model of an earthquake due to lack of data on the seismic ground-motion and seismic wave forms. Yet, by using the relations between earthquake moment magnitude $M_{\mathrm{w}}$ and seismic moment (e.g., $\mathcal{M}_{0}=16.05+1.5 M_{\mathrm{w}}$ by Hanks \& Kanamori (1979)) it is possible to estimate the seismic moment (Lay \& Wallace, 1995). In that case, the average slip $\bar{s}$ and the geometry of rupture plane are estimated using empirical relationships as also any evidence observed in the field, mostly at surface. For instance, Wells \& Coppersmith (1994) and Stirling et al. (2013) introduce empirical relationships for different tectonic settings and different faulting mechanisms. These relations associates the rupture geometry (e.g., rupture area $\mathcal{A}$, rupture length $\mathcal{L}$, observable rupture length on surface $\mathcal{L}_{s}$ and rupture width $\mathcal{W}$ ) and slip amounts (e.g., maximum slip, average slip and surface displacement) with earthquake magnitude $M$ or its seismic moment $\mathcal{M}_{0}$. Applying these relations along with field observations, one or few rupture geometries for the occurred earthquake can be proposed and the total, average and maximum slip can be estimated. Then, the slip should be distributed in a relevant way so that the total, average and maximum amounts of distributed slip values are close to the values obtained in field measurements and/or empirical relationships. The division of rupture plane into patches for distributing the slip values can be intuitive and experimental. One symmetrical distribution of slip, is the 2D ellipsoidal distribution with smoothed tapers on the edges that yields to a nearly constant stress drop inside the rupture area (Santoyo et al., 2005). In this approach, the slip is assumed to have an elliptical distribution with a cosine tapering of $10 \%$ to $15 \%$ over the length $\mathcal{L}$ and width $\mathcal{W}$ (Figure 2.15).
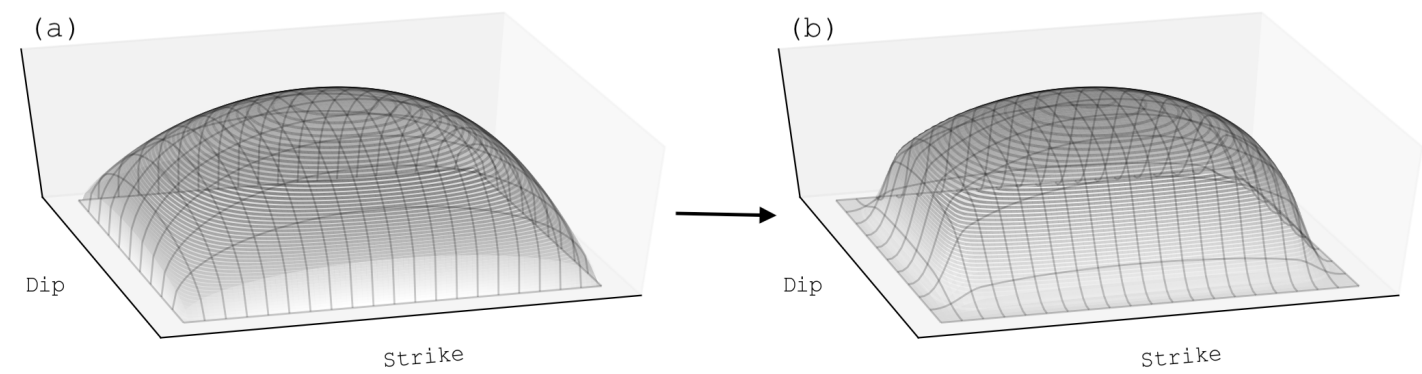

Figure 2.15: The 2D ellipsoidal distribution (a) and the same distribution with cosine tapering (b). 


\subsubsection{Coulomb failure stress change}

In an elastic half-space with the displacement field $\mathbf{U}(x, y, x)$, each point $(x, y, z)$ has a displacement given by $\left(u_{1} \hat{\mathbf{x}}_{1}, u_{2} \hat{\mathbf{x}}_{2}, u_{3} \hat{\mathbf{x}}_{3}\right)$, where $u_{i}$ describes the amount of displacement along direction $\hat{\mathbf{x}}_{i}$. The displacement differentials show different 3D displacements of neighbour points within infinitesimal volume $d V$ that lead to changes in distances and angles between them. For a volume $d V$, the change in length along $\hat{\mathbf{x}}_{i}$ and per length unit is called the normal strain $e_{i i}$. Considering a linear, elastic medium, the normal strain along $\hat{\mathbf{x}}_{i}$ is $e_{i i}=\frac{\partial u_{i}}{\partial x_{i}}$ (Stacey et al., 1977). Thus, the total amount of change in an unit of volume is $e=e_{11}+e_{22}+e_{33}$. In addition, the total angular change in the right-angle defined in the $\hat{\mathbf{x}}_{i} \hat{\mathbf{x}}_{j}$ plane is $\frac{\partial u_{i}}{\partial x_{j}}+\frac{\partial u_{j}}{\partial x_{i}}$. The shear strain $e_{i j}(i \neq j)$ is defined as the half of this angular change and $e_{i j}=e_{j i}$ (Stacey et al., 1977). For instance, if prior to the deformation, the position of points $\mathrm{D}, \mathrm{A}$ and $\mathrm{B}$ in $\hat{\mathbf{x}}_{1} \hat{\mathbf{x}}_{2}$ plane makes an angle of $\pi / 2$ (Figure 2.16) and after the deformation they make an angle of $\theta$, then the total shear strain in $\hat{\mathbf{x}}_{1} \hat{\mathbf{x}}_{2}$ plane is $2 e_{i j}=\pi / 2-\theta$. Indeed, strains are fractional changes (dimensionless) in the three dimensions of each volume element $d V$ inside the rock body that experiences the displacement field $\mathbf{U}$.

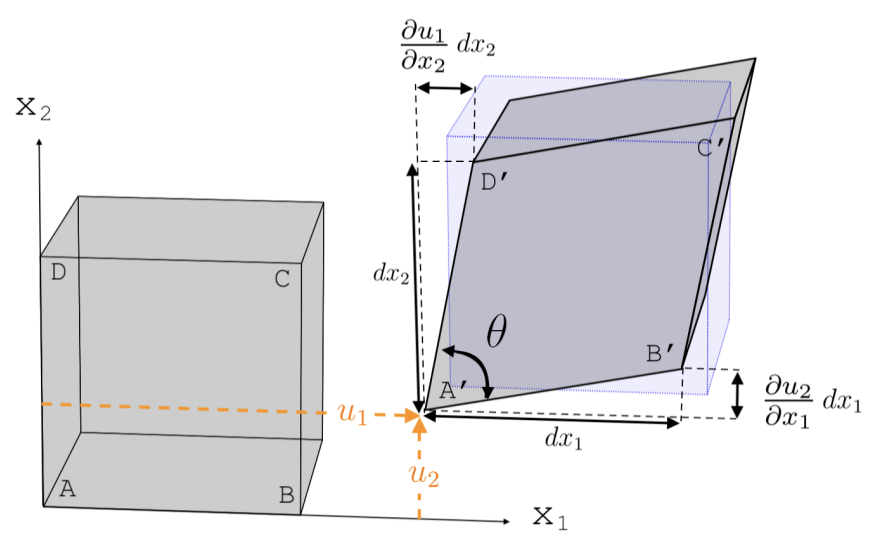

Figure 2.16: Displacements because of shear strains

For an isotropic material, two independent moduli are required to relate nine strain coefficients with nine stress coefficients and to conform the stress tensor. The definition of stress tensor is given in Box 2.5. This tensor defines the amount of normal and shear stress to the six sides of a volume element $d V$. The stress tensor prior to the displacement field $\mathbf{U}$ is called $\mathrm{T}$. The field $\mathrm{U}$ produces its own stress tensor $\mathrm{T}_{\mathrm{U}}$ that changes the overall stress state and can be estimated through elastic moduli (equation (2.65)).

$$
\begin{aligned}
\sigma_{11} & =\lambda e+2 \mathrm{G} e_{11} \\
\sigma_{22} & =\lambda e+2 \mathrm{G} e_{22} \\
\sigma_{33} & =\lambda e+2 \mathrm{G} e_{33} \\
\sigma_{12} & =\sigma_{21}=2 \mathrm{G} e_{12} \\
\sigma_{13} & =\sigma_{31}=2 \mathrm{G} e_{13} \\
\sigma_{23} & =\sigma_{32}=2 \mathrm{G} e_{23},
\end{aligned}
$$

where $\lambda$ is called Lamé parameter and can be estimated using elastic modulus G and Poisson's 
ratio $\nu$ as in equation (2.66) (Stacey et al., 1977) and:

$$
\lambda=\frac{2 \mathrm{G} \nu}{(1-2 \nu)} .
$$

Where $G$, is the rigidity or shear modulus and describes the ratio of shear stress to shear strain, therefore it has units of stress (pascals: $\mathrm{Nm}^{-2}$ ). The Poisson ratio or $\nu$ describes the change in the lateral ${ }^{9}$ dimension of the material. The Poisson ratio is given by the negative ratio of the lateral strain to the longitudinal strain.

Now, the stress tensor $\mathrm{T}_{\mathbf{U}}=\left[\sigma_{i j}\right]$ can be resolved onto any surface increment $\overrightarrow{d A}=d A \hat{\mathbf{n}}$ where, $d A$ is the area of the surface element and $\hat{\mathbf{n}}$ is the unit normal and outward to $d A$, thus:

$$
\mathrm{T}_{\mathbf{U}} \cdot \overrightarrow{d A}=\overrightarrow{\Delta \sigma_{s}}+\overrightarrow{\Delta \sigma_{n}}
$$

where $\overrightarrow{\Delta \sigma_{s}}$ and $\overrightarrow{\Delta \sigma_{n}}$ are respectively the shear stress and the perpendicular normal stress to $\overrightarrow{d A}$ ( $\Delta \sigma_{n}$ is positive for extension) produced by $\mathrm{T}_{\mathbf{U}}$. Applying the equation (2.64), the amount of change in the Coulomb failure stress $(\triangle \mathrm{CFS})$ due to the stress tensor $\mathrm{T}_{\mathrm{U}}$ can be estimated.

$$
\Delta \mathrm{CFS}=\Delta \tau_{0}+\mu_{f}\left(\Delta \sigma_{n}+\Delta \mathrm{p}_{\text {pore }}\right)
$$

where $\Delta \tau_{0}=\left|\Delta \sigma_{s}\right|$ or the absolute change in the shear stress for rupturing along same $\overrightarrow{\Delta \sigma_{s}}$ direction (zero angle with $\overrightarrow{\Delta \sigma_{s}}$ ). Hence, for a rupture (on plane $\overrightarrow{d A}$ ) in the direction that makes an angle of $\alpha$ with $\overrightarrow{\Delta \sigma_{s}}$ :

$$
\Delta \mathrm{CFS}=\Delta \tau_{\alpha}+\mu_{f}\left(\Delta \sigma_{n}+\Delta \mathrm{p}_{\text {pore }}\right)
$$

where $\Delta \tau_{\alpha}=\left|\Delta \sigma_{s}\right| \cos \alpha$, that can be positive or negative and the term $\left(\Delta \sigma_{n}+\Delta \mathrm{p}_{\text {pore }}\right)$ is the amount of change in extensional stress (positive if the compression is reduced and negative if the compression is increased). Indeed, the stress tensor $T_{U}$, can facilitate or not the shearing over $\overrightarrow{d A}$, and it depends on the shearing direction over $\overrightarrow{d A}$. Clearly, $\Delta \tau_{\alpha} \leq\left|\Delta \sigma_{s}\right|$, which means that the direction that has the most favourable shear stress change over $\overrightarrow{d A}$ is the $\overrightarrow{\Delta \sigma_{s}}$ direction. Accordingly $\triangle \mathrm{CFS}$ over $\overrightarrow{d A}$ will be maximum for a rupture along the $\overrightarrow{\Delta \sigma_{s}}$ direction.

Following a change in the stress perturbation within an isotropic poroelastic material, the pore pressure changes according to $\Delta \mathrm{p}_{\text {pore }}=-B \frac{\Delta \sigma}{3}$, in which $B$ is the Skempton coefficient for rock (Rice \& Cleary, 1976). $\sigma=\sigma_{11}+\sigma_{22}+\sigma_{33}$ is the volumetric stress, and if we assume that the fault zone materials are more ductile than the surrounding materials, then $\frac{\Delta \sigma}{3} \approx \Delta \sigma_{n}$ (Rice, 1992), thus we can rewrite equation (2.68) using the apparent coefficient of friction or $\mu_{f}^{*}=\mu_{f}(1-B)$ :

$$
\Delta \mathrm{CFS}=\Delta \tau_{\alpha}+\mu_{f}^{*}\left(\Delta \sigma_{n}\right)
$$

The coefficient $B$ theoretically ranges from 0 (for dry soil) to 1 (for fully saturated soil) (Skempton, 1954). Measurements show that $B$ varies between 0.47 to 1 and it is mostly reported to be between 0.7 and 1 (Harris, 2000). It is not easy to decide what value of $B$ to apply and relate the

\footnotetext{
${ }^{9}$ For uniaxial stress, lateral dimension is the dimension with no normal stress and the longitudinal dimension is the dimension with non-zero normal stress
} 
apparent friction to static friction over a fault (Scholz, 2019). The applied coefficient of friction $\mu_{f}$ in Coulomb stress studies is usually adopted form laboratory experimental values which are obtained for variety of rock types under different water content. For instance, Dieterich \& Conrad (1984) showed that dry conditions lead to rise a in $\mu_{f}$. Hence, according to the rock type and humidity condition of the target rupture, different coefficients of friction may be applied.

\section{$\Delta$ CFS for a specific earthquake rupture}

The stress tensor $\mathrm{T}_{\mathrm{U}}$ can be computed to evaluate the influence of earthquake $E Q 1$ for a given slip angle or rake $\lambda$ over a given plane $\overrightarrow{d A}$. A given rake over a given plane defines a hypothetical earthquake $E Q 2$. If the change in shear stress $\overrightarrow{\Delta \sigma_{s}}$, along a given rake $\lambda$ has a size of $\Delta \tau$ (can be positive or negative), then the change in the Coulomb failure stress ( $\triangle$ CFS) for the target earthquake $E Q 2$ is:

$$
\Delta \mathrm{CFS}=\Delta \tau+\mu_{f}^{*} \Delta \sigma_{n}
$$

If the cohesion $\mathbf{S}_{0}$ is constant over time, a positive change in CFS advances (in time) this hypothetical rupture of $E Q 2$ and a negative change in CFS delays it. The amount of this influence is more important when target earthquake $E Q 2$ is close to the earthquake $E Q 1$, because close to a ruptured fault, the ratio of the regional tectonic stress is comparable with $\triangle \mathrm{CFS}$. Far from earthquake $E Q 1$, the occurrence of hypothetical earthquake $E Q 2$ is more controlled by the regional stress and state of the $E Q 2$ 's rupture plane within its interseismic cycle. However, if $E Q 2$ stays very close to its natural failure point, static stress changes as low as 0.1 bar can trigger it (Reasenberg \& Simpson, 1992; King et al., 1994).

This method is commonly used for understanding the influence of an earthquake in triggering other earthquakes nearby, such as an offspring or aftershock with known focal mechanism. Nevertheless, most of the aftershock population have relatively small magnitudes with no available data about their focal mechanism. King et al. (1994) explains that if there are enough number of faults in all orientations, the small aftershocks are most likely to occur on optimally orientated faults. Hence, it is common to explore the influence of earthquake EQ1 on $\triangle \mathrm{CFS}$ over the optimum rupture orientations too.

\section{$\triangle$ CFS for optimum ruptures}

Faults are surfaces of weakness where the physical condition along them makes their rupture easier. Fault orientations are the result of tectonic stresses, which directions usually persist during millions of years. These tectonic movements have caused numerous ruptures on optimally oriented planes which can be active or not today (Stacey et al., 1977). Those faults that are no longer active, indicate the direction of tectonic stresses that caused their failures many years ago. An optimally oriented plane $\overrightarrow{d A}_{\text {opt }}$ in a confined rock, is a plane for which the amount of CFS is maximum and the shear stress would overcome the cohesion on this plane before along any other plane. Equation (2.64) describes that the rupture occurs over such $\overrightarrow{d A}_{\text {opt }}$, if CFS $\geq \mathbf{S}_{\mathbf{0}}$ or:

$$
\left|\sigma_{s}\right|+\mu_{f}^{*} \sigma_{n} \geq \mathbf{S}_{\mathbf{0}}
$$

It is known from laboratory experiments, that a confined rock under principal stresses $\sigma_{1}, \sigma_{2}$ and $\sigma_{3}$ (where $\sigma_{1}$ is the maximum, $\sigma_{2}$ the intermediate and $\sigma_{3}$ is the minimum principal stresses) 
breaks over a plane parallel to the $\sigma_{2}$ axis, because it experiences the largest shear stress (Stacey et al., 1977). The definition of principal stresses and their representative axes $\hat{\mathbf{x}}_{1}^{\prime}, \hat{\mathbf{x}}_{2}^{\prime}$ and $\hat{\mathbf{x}}_{3}^{\prime}$ are given in Box 2.6.

The angle that an optimum rupture plane makes with the axes of $\sigma_{1}$ and $\sigma_{3}$, directly depends on the effective coefficient of friction $\mu_{f}^{*}$. Figure 2.17 shows the shear and normal stresses on a plane that has a positive angle ${ }^{10}$ of $\beta$ with the direction of $\hat{\mathbf{x}}_{1}$ in both compression-positive and extension-positive coordinate systems.
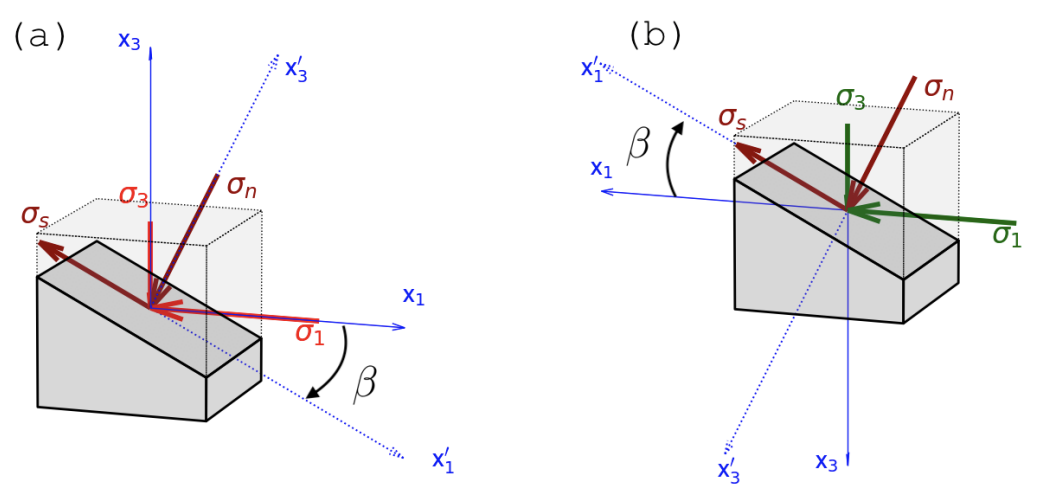

Figure 2.17: Shear and normal stresses on a plane that has a positive angle of $\beta$ with the direction of $\hat{\mathbf{x}}_{1}$ for extension-positive (a), and compression-positive (b) coordinate systems.

\section{Box 2.6}

\section{Principal stresses}

For any stress tensor in an isotropic material, it is possible to find three orthogonal unit areas n1, $\hat{\mathbf{n} 2}$ and $\hat{\mathbf{n} 3}$ across which, the stress tensor impose no tangential components or:

$$
\text { Thì }=\hat{l n i}
$$

that leads to solving the cubic equation below:

$$
\left|\begin{array}{ccc}
\sigma_{11}-l & \sigma_{12} & \sigma_{13} \\
\sigma_{21} & \sigma_{22}-l & \sigma_{23} \\
\sigma_{31} & \sigma_{32} & \sigma_{33}-l
\end{array}\right|=0 .
$$

The result of the above equation gives three values for $l$ that are: $l_{1}, l_{2}$ and $l_{3}$. Applying $l_{1}$ into equation (B.73), together with the property of unit area vector for $\hat{\mathbf{n}} \mathbf{1}$ or: $n 1_{1}^{2}+$ $n 1_{2}^{2}+n 1_{3}^{2}=1$, we can find $\hat{\mathbf{n} 1}$. The same way we can find $\hat{\mathbf{n} 2}$ and $\hat{\mathbf{n} 3}$. These three unit areas where tensor $\mathrm{T}$ have only normal component across them, are indicating the three principal stress directions in the material (Stacey et al., 1977). Vectors ni are usually denoted in a way that $\left|\overrightarrow{\mathbf{T}}_{\hat{\mathbf{n}} 1}\right|>\left|\overrightarrow{\mathbf{T}}_{\hat{\mathbf{n}} 2}\right|>\left|\overrightarrow{\mathbf{T}}_{\hat{\mathbf{n}} 3}\right|$. Thus, if the Cartesian coordinate system $\left(\hat{\mathbf{x}}_{1}, \hat{\mathbf{x}}_{2}, \hat{\mathbf{x}}_{3}\right)$ is transformed into a new coordinate system with unit vectors $\left(\hat{\mathbf{x}}_{1}^{\prime}=\hat{\mathbf{n}}\right.$,

\footnotetext{
${ }^{10}$ Based on the right-hand rule for rotations around $\hat{\mathbf{x}}_{2}$, a positive angle in $\hat{\mathbf{x}}_{1} \hat{\mathbf{x}}_{3}$ plane is clockwise
} 
$\hat{\mathbf{x}}_{2}^{\prime}=\hat{\mathbf{n} 2}, \hat{\mathbf{x}}_{3}^{\prime}=\hat{\mathbf{n} 3}$ ), the transformed stress tensor $\mathrm{T}$ is:

$$
\mathbf{T}^{\prime}=\left[\begin{array}{ccc}
\sigma_{1} & 0 & 0 \\
0 & \sigma_{2} & 0 \\
0 & 0 & \sigma_{3}
\end{array}\right]
$$

Tensor $T^{\prime}$ is called principal stress tensor.

\section{Regional principal stress}

There are different ways that can be applied for estimating the orientation of regional principal stresses. The world stress map (WSM) project ${ }^{\mathrm{I}}$ compiles information about crustal stress field over the world and provides a global map with the regional stress orientation (Figure 2.18).

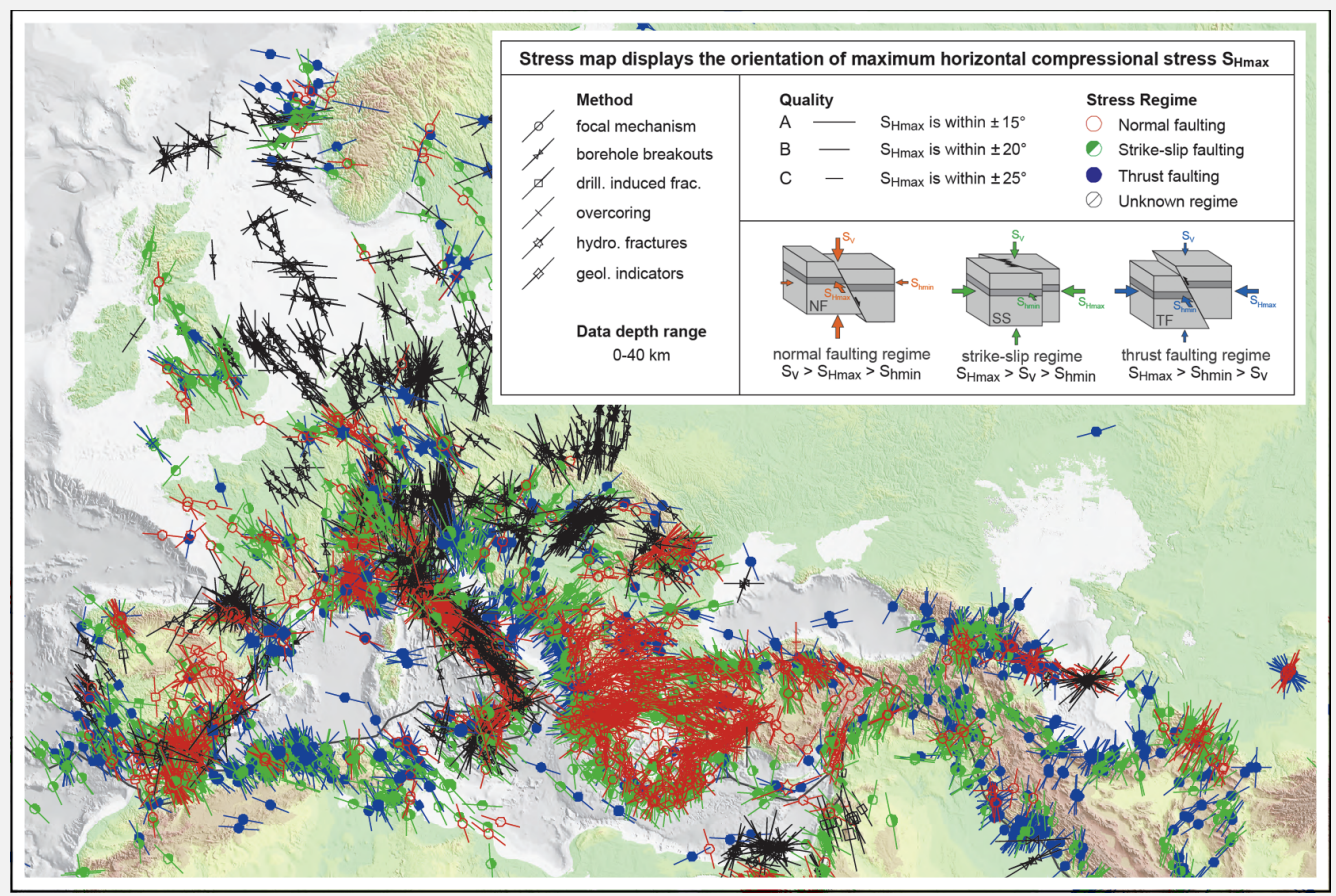

Figure 2.18: A cropped image form the latest production of WSM project which was published in 2016 (Heidbach et al., 2016, 2018). As it is shown in the legend, different type of data were used for extracting the information on the regional stress orientations.

\footnotetext{
Ihttp://www.world-stress-map.org
}

Regardless of the coordinate system, the normal stress to the plane is compressional with value of $\left(\frac{\sigma_{1}+\sigma_{3}}{2}-r \cos 2 \beta\right)$ and the shear stress on this plane is $r \sin 2 \beta$ (see Box 2.7), where $r=\frac{\sigma_{1}-\sigma_{3}}{2}$. The Mohr circle (Figure 2.19) is a diagram for expressing the normal and shear stresses across a hypothetical rupture plane, with respect to the magnitude and direction of principal stresses. The Mohr circle in Figure 2.19 encapsulates the size and direction of stresses in Figure 2.19b. Moreover, this diagram shows that the Coulomb condition can be fulfilled on two specific planes. One has a positive angle of $\beta_{1}$ with $\hat{\mathbf{x}}_{1}$ resulting a positive shear stress on this plane. The other 
one has a negative angle of $\beta_{2}=-\beta_{1}$ and results a negative shear stress on it. For both planes the absolute size of shear stress is the same $\left|\sigma_{s}\right|=\mathbf{S}_{\mathbf{0}}+\mu_{f}^{*} \sigma_{n}\left(\sigma_{n}\right.$ is positive) and its sign does not affect the amount of CFS but it determines the direction of slip over the rupture plane. The Mohr circle in Figure 2.19 shows that the geometrical solution for $\beta$ which fulfils the Coulomb condition over $\overrightarrow{d A}_{\text {opt }}$ is:

$$
\beta=\frac{1}{2}\left(\frac{\pi}{2}-\tan ^{-1} \mu_{f}^{*}\right)
$$

and it only depends on the effective coefficient of friction $\mu_{f}^{*}$.

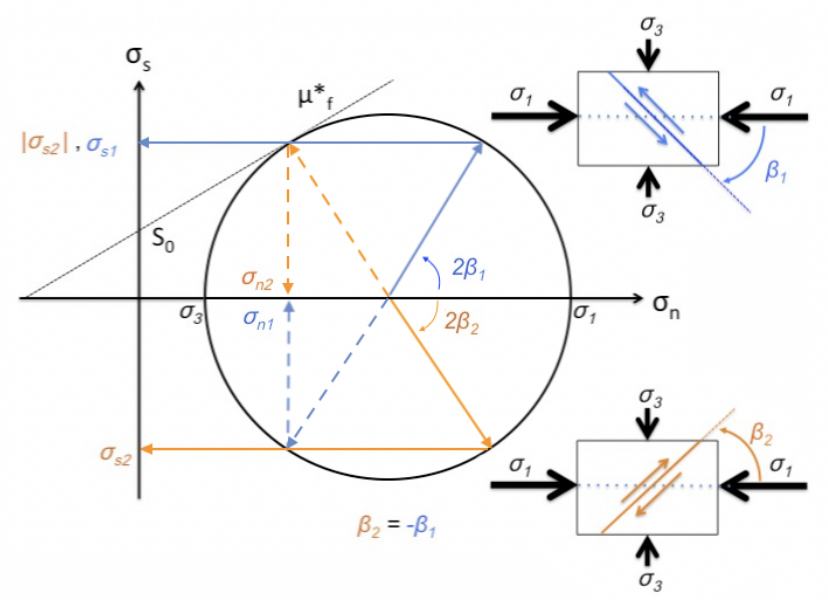

Figure 2.19: The Mohr circle can be used for showing the amount of shear and normal stresses for different plane orientations. The positive rotation in the Mohr circle is always anticlockwise.

For the extension-positive system the circle and black line in Figure 2.19 has to be mirrored with respect to the $\sigma_{s}$ axis. The Coulomb condition for extension-positive system implies $\left|\sigma_{s}\right| \geq \mathbf{S}_{\mathbf{0}}-\mu_{f}^{*} \sigma_{n}$ (same as equation (2.72)).

Anderson (1905) summarized these conclusions for classifying different faulting criteria. In his classification, the absence of normal and shear stresses across a free horizon surface is basic. This implies that one of the principal stress directions is vertical. Sibson \& Xie (1998) and Jackson \& White (1989) used the Anderson criteria and applied the relation (2.73) for different coefficients of friction to determine optimum faulting mechanism in regions where one of the principal stress directions in vertical.

Indeed, finding the optimum rupture planes in an area, requires data on principal stress directions. One of the methods for determining the regional tectonic stress directions (see Box 2.6) is analysing the faulting mechanism in the zone. However, when an earthquake occurs, at very close distances to its ruptured area, the associated $T_{U}$ produces very high stresses of about the size of the regional stress. Therefore, for regions that are highly influenced by pre-existing displacement fields, the principal stress directions have to be recalculated.

Finally, by estimating the optimum rupture orientations in an area, it is possible to evaluate the influence of earthquake $E Q 1$ in $\triangle \mathrm{CFS}$ along them. Thus, $\triangle \mathrm{CFS}$ is calculated for $\boldsymbol{\lambda}=90^{\circ},-90^{\circ}$, $0^{\circ}$ and $180^{\circ}$ on the normal, reverse, right-lateral and left-lateral strike-slip rupture mechanism 
respectively. Such estimations help us analysing the spatial distribution of aftershocks. It has been observed that the aftershock population is increased in regions of positive $\triangle$ CFS and is decreased in regions of negative $\Delta$ CFS (e.g., Reasenberg \& Simpson, 1992; Toda et al., 1998).

\section{Box 2.7}

\section{Stress tensor for optimum ruptures}

The stress tensor acting on a plane $\overrightarrow{d A}$ parallel to $\hat{\mathbf{x}}_{2}^{\prime}$, where the plane constructs an angle of $\beta$ with $\hat{\mathbf{x}}_{1}^{\prime}$, can be derived by rotating principal matrix $\mathrm{T}^{\prime}$ around $\hat{\mathbf{x}}_{2}^{\prime}$ by an angle of $\beta$. In general:

$$
\mathrm{T}_{i}^{\prime \prime}=R_{i} \cdot \mathrm{T}^{\prime} \cdot R_{i}^{T}
$$

where $R_{i}$ is the rotation matrix for positive rotation around $\hat{\mathbf{x}}_{i}^{\prime}$, and $\mathrm{T}_{i}^{\prime \prime}$ is the transpose stress tensor. A positive rotation around $\hat{\mathbf{x}}_{2}^{\prime}$ with $R_{2}=\left[\begin{array}{cc}\cos \beta & -\sin \beta \\ \sin \beta & \cos \beta\end{array}\right]$ yields:

$$
\mathrm{T}_{2}^{\prime \prime}=R_{2} \cdot\left[\begin{array}{cc}
\sigma_{1} & 0 \\
0 & \sigma_{3}
\end{array}\right] \cdot R_{2}^{T}=\left[\begin{array}{cc}
\frac{\sigma_{1}+\sigma_{3}}{2}+r \cos 2 \beta & r \sin 2 \beta \\
r \sin 2 \beta & \frac{\sigma_{1}+\sigma_{3}}{2}-r \cos 2 \beta
\end{array}\right],
$$

where $r=\frac{\sigma_{1}-\sigma_{3}}{2}, \sigma_{n}=\left(\frac{\sigma_{1}+\sigma_{3}}{2}-r \cos 2 \beta\right)$ and $\sigma_{s}=r \sin 2 \beta$.

A positive rotation around $\hat{\mathbf{x}}_{1}^{\prime}$ with $R_{1}=\left[\begin{array}{cc}\cos \beta & \sin \beta \\ -\sin \beta & \cos \beta\end{array}\right]$ gives $\sigma_{n}=\left(\frac{\sigma_{2}+\sigma_{3}}{2}-r \cos 2 \beta\right)$ and $\sigma_{s}=-r \sin 2 \beta$, where $r=\frac{\sigma_{2}-\sigma_{3}}{2}$.

A positive rotation around $\hat{\mathbf{x}}_{3}^{\prime}$ with $R_{3}=\left[\begin{array}{cc}\cos \beta & \sin \beta \\ -\sin \beta & \cos \beta\end{array}\right]$ yields $\sigma_{n}=\frac{\sigma_{1}+\sigma_{2}}{2}-r \cos 2 \beta$ and $\sigma_{s}=-r \sin 2 \beta$, where $r=\frac{\sigma_{1}-\sigma_{2}}{2}$. The state of stress at depth in the Earth is nearly always compressional, and therefore all three normal principal stresses are negative in an extension-positive system Lay \& Wallace (1995).

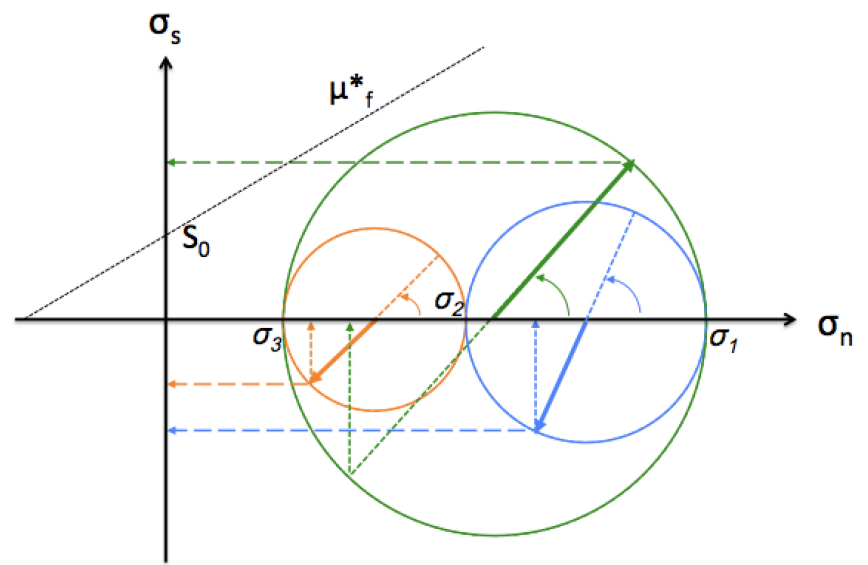

Figure 2.20: The Mohr circle for the compression-positive system. For the extension-positive system the circles and the black line has to be mirrored with respect to the $\sigma_{s}$ axis. The positive angle in the Mohr circle is always anticlockwise and the sign of $r$ is respected (presented in bold arrow) for finding the normal and shear stresses (dotted arrows and dashed arrows respectively). 
Figure 2.20 shows the relations above for a compression-positive system with the Mohr diagram. This figure shows that the shear stress over a plane parallel to $\hat{\mathbf{x}}_{2}^{\prime}$ can gets larger in comparison with shear stresses over planes parallel to $\hat{\mathbf{x}}_{1}^{\prime}$ or $\hat{\mathbf{x}}_{3}^{\prime}$. For an extension-positive system the circle and black line has to be mirrored with respect to the $\sigma_{s}$ axis. In any case, the positive rotation is always anticlockwise and the sign of $r$ determines the sign of shear stress.

\subsection{Probabilistic seismic hazard estimation}

Probabilistic seismic hazard assessment searches for the exceedance probabilities of a strong ground motion level in a given exposure time. In section 1.2.4, the general idea about the exceedance rate of the strong ground motion in unit of time is described.

This rate $\Lambda$ gives the number of times that the ground motion $\psi$ exceeds some specific level $\psi^{*}$, in an unit of time. If this number of exceedances only depends on the duration of the exposure interval $\Delta t$ and not on the starting and ending time of this period, then for small $\Lambda \Delta t$, the probability for the number of exceedances being equal to $\mathrm{K} \in \mathbb{N}$ is given by the stationary Poisson distribution (for mathematical proofs see "Stationary rate" in section 2.1.2):

$$
P(\mathrm{~K}=k)=\frac{(\Lambda \Delta t)^{k} e^{-\Lambda \Delta t}}{k !} .
$$

The probability of at least one exceedance in $t$ unit of time is $P(\mathrm{~K}>0)=1-P(\mathrm{~K}=0)$, hence:

$$
P\left(\psi>\psi^{*}\right)=1-e^{-\Lambda \Delta t} .
$$

If the exposure time is equal to unit of time and $\Lambda \ll 1$, then $P\left(\psi>\psi^{*}\right)=1-e^{-\Lambda} \cong \Lambda$. In another words, for a stationary exceedance rate, if the exceedance rate is very small, the probability of exceedance in unit of time is equal to the exceedance rate. However, most of the time we search for the seismic hazard assessments in larger exposure time (years, decades or more), so we actually require that $\Lambda \Delta t \ll 1$ to reach $P\left(\psi>\psi^{*}\right) \cong \Lambda \Delta t$.

The occurrence of an earthquake sequence updates the earthquake catalog. The added data might involve a change in the long-term average of number of events in a unit of time or earthquake rate $\lambda$. Moreover, an earthquake sequence usually produces non-uniform variations in the earthquake density rates for different magnitudes $\dot{\rho}(m, x, y, z)$, where $x, y$ and $z$ are the spatial coordinates of the seismogenic area. The implication of these changes for probabilistic seismic hazard can be considered through updated estimation of $\Lambda\left(\psi>\psi^{*}\right)$.

In that regard, a zone-less approach is applied in this dissertation to estimate the exceedance probability $\Lambda\left(\psi>\psi^{*}\right)$ in equation (1.6). The basic methodology is adopted from Woo (1996a) and I programmed the computations in Python (named as GEREK1.0 ${ }^{11}$ program). I hereby explain the algorithm in which I used, though modified, the KERFRACT program by Woo (1996b).

\footnotetext{
${ }^{11}$ Ground motion Exceedance Rate Estimation using Kernel function for the spatial distribution of earthquake
} 


\subsubsection{Earthquake epicentre density rate estimation}

Initially, earthquake density rates associated with the earthquake activity inside a limited area $\Phi$ and during a limited period of time $T$, are calculated priorly. For that purpose, the methodology in section 2.1.5 is used and the magnitude and epicentre uncertainties are also implemented in the calculations.

The continuous magnitude range of the earthquakes $\left[m_{c}, m_{\max }\right]$ is discretized into $J$ magnitude classes $\mathbb{M}_{J}=\left\{m_{1}, m_{2}, \ldots, m_{J}\right\}$, where each magnitude class $m_{j}$ stands for all magnitudes inside $\left[m_{j}-\frac{\Delta m}{2}, m_{j}+\frac{\Delta m}{2}\right)$ and is associated with given completeness period $T_{m_{j}} \leq T$ for which events with magnitude $m_{j}$ are fully recorded. The number of events with magnitude class $m_{j}$ during $T$ units of time are given by $\mathbb{N}_{J}=\left\{n_{m_{1}}, n_{m_{2}}, \ldots, n_{m_{J}}\right\}$. Therefore, there are $n_{m_{j}}$ earthquakes with magnitude $m_{j}$ and epicentral coordinates $\left(E X_{j i}, E Y_{j i}\right)$, where $i=1,2, \ldots$, $n_{m_{j}}$. Applying equation (2.42), the total earthquake epicentre density rate for magnitude $m_{j}$ at coordinates $(x, y)$ is:

$$
\dot{\rho}\left(m_{j}, x, y\right)=\frac{\sum_{i=1}^{n_{m_{j}}} \mathbf{K}\left(\mathrm{x}_{j i}, \mathrm{y}_{j i} \mid m_{j}\right)}{T_{m_{j}}},
$$

where $\mathrm{x}_{j i}=x-E X_{j i}$ and $\mathrm{y}_{j i}=y-E Y_{j i}$. For the bivariate Kernel function $\mathbf{K}$, the function in equation (2.51) is used. This function computes the spatial probability density of an earthquake with exact magnitude of $m_{j}$ at exact epicentre coordinates of $\left(E X_{j i}, E Y_{j i}\right)$.

However, both magnitude $m_{j}$ and epicentral coordinates $\left(E X_{j i}, E Y_{j i}\right)$ of each event $E_{j i}$ in the earthquake catalog have uncertainties to consider in the estimation of $\dot{\rho}\left(x, y, m_{j}\right)$. These uncertainness are usually related to the quality of seismic instruments and their spatiotemporal coverage. Next, it is explained how the Kernel function $\mathbf{K}$ is used to compute the earthquake density rates taking into account the catalog errors in the estimation of magnitude and epicentre coordinates:

\section{- Epicentre error implementation:}

The epicentre errors in an earthquake catalog are usually given as $\pm \Delta_{E X}$ along longitude and $\pm \Delta_{E Y}$ along latitude. To take into account these uncertainties, nine probable coordinates are considered at combination of $\left\{E X_{j i}-\Delta_{E X}, E X_{j i}, E X_{j i}+\Delta_{E X}\right\}$ and $\left\{E Y_{j i}-\Delta_{E Y}, E Y_{j i}, E Y_{j i}+\Delta_{E Y}\right\}$ for longitude and latitude respectively (e.g., event $E_{j 2}$ in Figure 2.21) shows these nine possibilities. The earthquake spatial probability density function at $(x, y)$ and due to event $E_{j i}$ is estimated as the average of the Kernel value for all nine possibilities:

$$
p d f\left(\mathrm{x}_{j i}, \mathrm{y}_{j i} \mid m_{j}\right)=\sum_{l=1}^{9} \mathbf{K}\left(\mathrm{x}_{j i l}, \mathrm{y}_{j i l} \mid m_{j}\right) / 9 .
$$

The relation above gives the probability density function that the earthquake $E_{i}$ with exact magnitude $m_{j}$ is located at distance $\mathrm{x}_{j i}$ along longitude, $\mathrm{y}_{j i}$ along latitude. Nevertheless, the earthquake magnitude is not an exact value and its estimation involves systematic errors. 


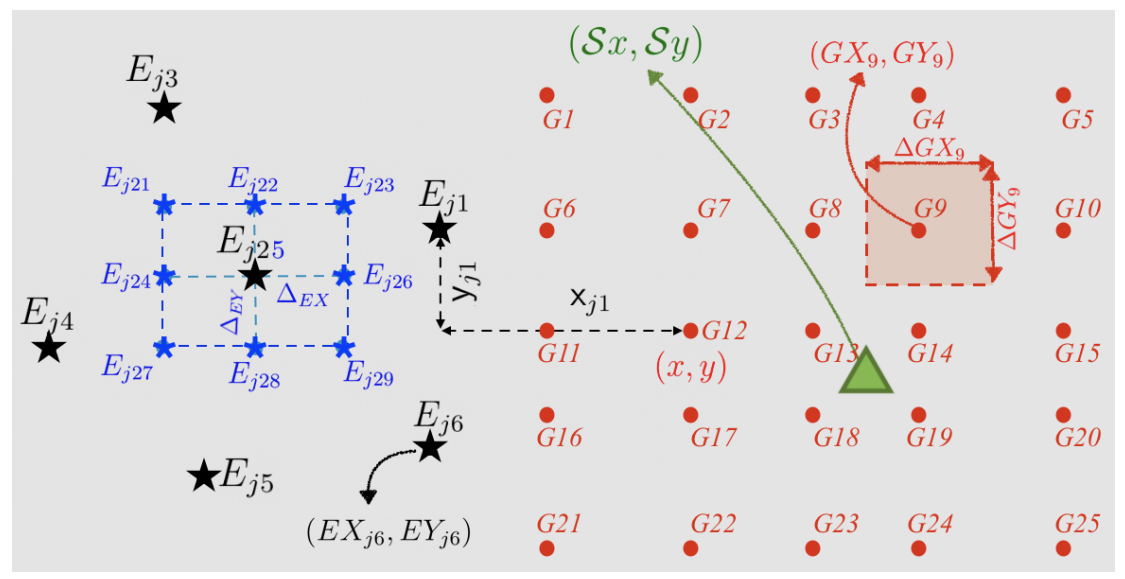

Figure 2.21: The black stars presents an example of six events with magnitude $m_{j}$, where the epicentre error and other eight probable location of event $E_{j 2}$ are shown in blue stars. The red dot presents an example of 25 grid points $G_{k}$ around calculation site $\mathcal{S}$ shown as a green triangle. The coordinate $(x, y)$ is equation (2.79) is set for $G 12$ as an example. The increment area related to grid point $G 9$ is shown as an instance.

\section{- Magnitude error implementation:}

The magnitude $m_{j}$ usually have normal distribution $f_{m_{j}, \sigma_{m}}(m)=\frac{1}{\sqrt{2 \pi \sigma_{m}^{2}}} \exp \left(\frac{-\left(m-m_{j}\right)^{2}}{2 \sigma_{m}^{2}}\right)$. The probability that a given magnitude $m$ in the earthquake catalog represents an event with magnitude $m_{j}$ is equal to $f_{m_{j}, \sigma_{m}}(m) \Delta m$. This probability has to be multiplied with the spatial probability density function at $(x, y)$ and due to event $E_{j i}$. Thus, the probability density function that the earthquake $E_{i}$ is located at distance $\mathrm{x}_{j i}$ along longitude, $\mathrm{y}_{j i}$ along latitude and its magnitude is $m_{j}$ is:

$$
p d f\left(\mathrm{x}_{j i}, \mathrm{y}_{j i}, m_{j}\right)=p d f\left(\mathrm{x}_{j i}, \mathrm{y}_{j i} \mid m_{j}\right) f_{m_{j}, \sigma_{m}}\left(m_{j}\right) \Delta m
$$

The estimation of density rates is carried out separately for each magnitude class $m_{j}$. To do that, a range of magnitudes $m_{j h}=\left\{m_{j} \pm \eta \Delta m\right\}$ is considered to contribute $(\eta \in \mathbb{N})$. If we set $\eta=2$, then we have $h=1,2, \ldots, 5$ and the density rate for the magnitude class $m_{j}$ at $(x, y)$ is:

$$
\begin{aligned}
\dot{\rho}\left(x, y, m_{j}\right)= & \sum_{h=1}^{5} \frac{\sum_{i}^{n_{m_{j h}}} p d f\left(\mathrm{x}_{j h i}, \mathrm{y}_{j h i}, m_{j h}\right)}{T_{m_{j h}}} \\
& =\sum_{h=1}^{5} \frac{\sum_{i}^{n_{m_{j h}}}\left[f_{m_{j}, \sigma_{m}}\left(m_{j h}\right) \Delta m \sum_{l=1}^{9}\left[\mathbf{K}\left(\mathrm{x}_{j h i l}, \mathrm{y}_{j h i l} \mid m_{j h}\right) / 9\right]\right]}{T_{m_{j h}}}
\end{aligned}
$$

After the estimation of density rates for all magnitude increments in $\mathbb{M}_{J}$, the GEREK1.0 program enters to the next step for estimation of exceedance rate of the strong ground motion $\psi^{*}$.

\subsubsection{Exceedance rate of the strong ground motion}

To find the exceedance probability of the strong ground motion $\psi$ from a specific value $\psi^{*}$ in a calculation site at $(\mathcal{S} x, \mathcal{S} y)$, we use a ground motion prediction equation (GMPE). These 
equations describe the attenuation of the ground motion due to an earthquake with magnitude $m_{j}$ with earthquake-to-site distance of $r_{k}$.

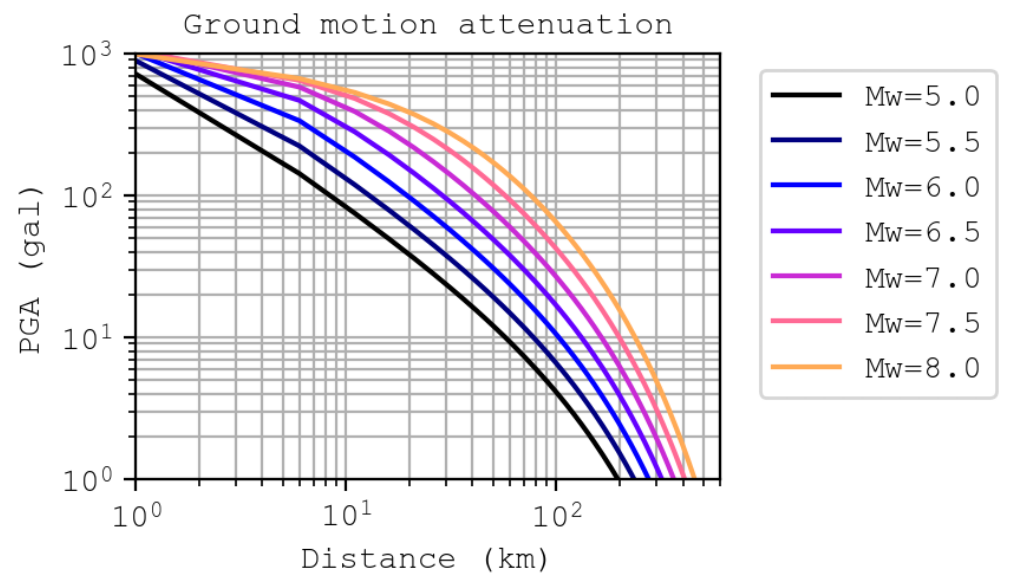

Figure 2.22: The attenuation of peak ground acceleration (PGA) by distance and for magnitudes between $M_{\mathrm{w}} 5.0$ and $M_{\mathrm{w}} 8.0$ using the ground motion prediction equation proposed by Arroyo et al. (2010) for Mexican interplate earthquakes.

Around each computation site a rectangular grid of $n_{G}$ points $\left(G X_{k}, G Y_{k}\right)\left(k=1,2, \ldots, n_{G}\right)$ is considered with a grid point separation of $\Delta G X_{k}$ and $\Delta G X_{k}$ along longitude and latitude respectively. For each grid point, the associated depth $Z_{k}$ on the source is determined and then the distance of $\left(G Y_{k}, G Y_{k}, Z_{k}\right)$ to the site is calculated as $r_{k}=\left[\left(G X_{k}-\mathcal{S} x\right)^{2}+\left(G Y_{k}-\mathcal{S} y\right)^{2}+\right.$ $\left.Z_{k}^{2}\right]^{\frac{1}{2}}$. Sometimes the source does not comprise specific geometry (e.g., subduction interface in GEREK1.0 program) (e.g., a source that comprises all the crustal earthquakes in an area and down to some specific depth without associating the earthquakes to known faults or rupture planes). Therefore, the depth of grid points $Z_{k}$, is determined with respect to the magnitude class $m_{j}$ for which we are estimating the ground motion. A histogram of depth distribution versus magnitude is shown in Figure 2.23. For each magnitude class $m_{j}$, the average depth $\bar{Z}_{m_{j}}$ can be used as $Z_{k}$ of all the grid points.

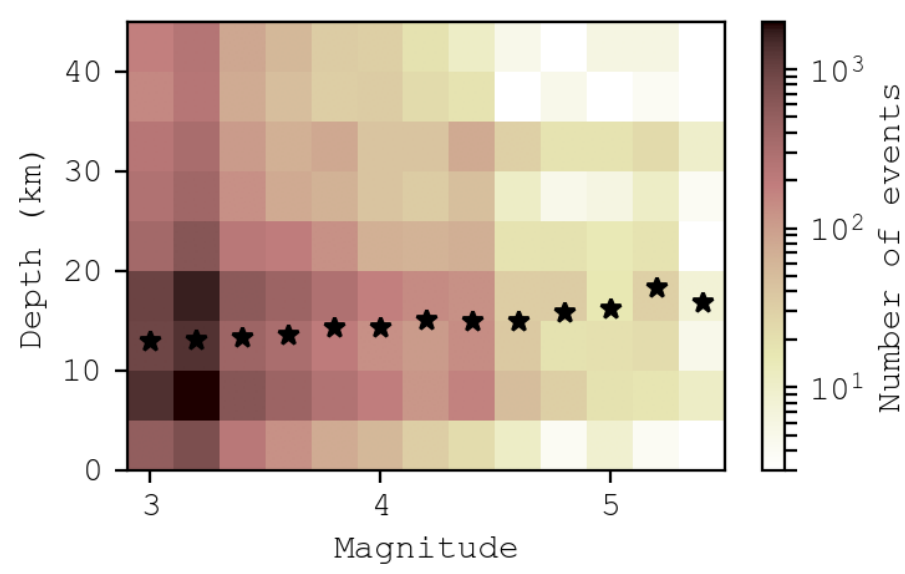

Figure 2.23: Depth distribution versus magnitude for shallow earthquakes (depth $\leq 45 \mathrm{~km}$ ) with magnitudes between $M_{\mathrm{w}} 2.9$ and $M_{\mathrm{w}} 5.5$ and within latitudes $\left[15^{\circ}, 20^{\circ}\right]$ and longitudes $\left[96^{\circ}, 105^{\circ}\right]$ in Mexican catalog. The black stars show the average depth versus magnitude. 
In order optimize the program runtime, the dimension of our grid is magnitude-dependent. The Figure 2.22 shows how the peak ground acceleration (PGA) decreases with hypocentral distance for different magnitudes. The effective distance to reach a significant strong ground motion (e.g., PGA $\sim 0.01 \mathrm{~g}$ Wald et al., 1999) in the Earth surface depends on the magnitude. In GEREK1.0 program, the grid is expanded equally along latitude and longitude up to distances of $\pm 40, \pm 50$, $\pm 80, \pm 100$ and $\pm 200 \mathrm{~km}$ for magnitude ranges $4.5<m_{j} \leq 5.0,5.0<m_{j} \leq 5.5,5.5<m_{j} \leq 6.0$, $6.0<m_{j}<6.5$ and $6.5 \leq m_{j}$ respectively.

Applying an appropriate ground motion prediction equation (GMPE), the strong ground motion for magnitude $m_{j}$ at hypocentral distance $r_{k}$ is estimated: $\Psi_{j k}$. This value either exceeds $\psi^{*}$ or not. However, GMPE are empirical models which error is usually represented by a log-normal distribution with an associated $\sigma_{\psi}$ (equation 1.3) that is given together with the model parameters. Applying this error, the exceedance probability of $\psi^{*}$ is:

$$
\begin{aligned}
P\left(\Psi_{j k}>\psi^{*}\right)= & \int_{\psi^{*}}^{\infty} f_{\Psi_{j k}, \sigma_{\psi}}(\psi) d \psi \\
& =\int_{\psi^{*}}^{\infty} \frac{1}{\sqrt{2 \pi \sigma_{\psi}^{2}}} \exp \left(-\frac{\left(\psi-\Psi_{j k}\right)^{2}}{2 \sigma_{\psi}^{2}}\right) d \psi
\end{aligned}
$$

and the exceedance rate of $\psi^{*}$ because of earthquakes with magnitude $m_{j}$ can be computed:

$$
\Lambda\left(\psi>\psi^{*} \mid m_{j}\right)=\sum_{k=1}^{n_{G}} P\left(\Psi_{j k}>\psi^{*}\right) \dot{\rho}\left(G X_{k}, G Y_{k}, m_{j}\right) \cdot \Delta A_{k}
$$

where $\dot{\rho}\left(G X_{k}, G Y_{k}, m_{j}\right)$ is the epicentral density rate of magnitude class $m_{j}$ at each grid point $\left(G X_{k}, G Y_{k}\right)$ and $\dot{n}\left(m_{j}, G X_{k}, G Y_{k}, Z_{k}\right)=\dot{\rho}\left(G X_{k}, G Y_{k}, m_{j}\right) \cdot \Delta A_{k}$, where $\Delta A_{k}$ is the area size of source element associated to the point $\left(G X_{k}, G Y_{k}, Z_{k}\right)$. If the source does not comprise geometry in 3D space then $d A_{k}=\Delta G X_{k} \cdot \Delta G Y_{k}$, otherwise this area can easily be estimated by $\left(\Delta G X_{k} \cdot \Delta G Y_{k}\right) / \cos (\delta)$, where $\delta$ is the dip angle of the source element (e.g., the angle that subduction interface area element constructs with the surface). Considering that some GMPE apply epicentral distance and depth as separate inputs, the algorithm in GEREK1.0, same as in KERFRACT program by Woo (1996b), first estimates the exceedance probability $P\left(\Psi_{j k}>\psi^{*}\right)$ associated to earthquakes over $2 \mathrm{D}$ surface elements, though in 3D space, and next it incorporates the systematic errors in depth estimations as explained below:

\section{- Depth error implementation:}

Depth errors in an earthquake catalog are usually given as $\pm \Delta_{E Z}$. To count these uncertainties, three depths are considered at combination of $Z_{k l}=\left\{Z_{k}-\Delta_{E Z}, Z_{k}, Z_{k}+\Delta_{E Z}\right\}$.

$$
P\left(\Psi_{j k}>\psi^{*}\right)=\sum_{l=1}^{3} P\left(\psi>\psi^{*} \mid m_{j}, r_{k l}\right) / 3
$$

In GEREK1.0 program for each grid point $\left(G X_{k}, G Y_{k}\right)$, the depth of the seismicity is the subduction interface depth. All the earthquake used for estimation of $\dot{\rho}\left(G X_{k}, G Y_{k}, m_{j}\right)$ are associated to the subduction interface seismicity. To select these earthquakes all catalog 
hypocentres within +5 and $-15 \mathrm{~km}$ vertical distance to the subduction interface are picked up and they are all projected into the surface for estimation of subduction earthquakes density rates. Therefore instead of using relation (2.82), I associate the estimated density rates to four depth values $Z_{k l}=\left\{Z_{k}-5, Z_{k}, Z_{k}+5, Z_{k}+15\right\} \mathrm{km}$ and weighted them with $w_{l}=\{25 \%, 35 \%, 25 \%, 15 \%\}$ respectively to count the depth error for subduction interface earthquakes.

$$
P\left(\Psi_{j k}>\psi^{*}\right)=\sum_{l=1}^{4} P\left(\psi>\psi^{*} \mid m_{j}, r_{k l}\right) w_{l}
$$

Finally, the total exceedance rate of the strong ground motion is the sum of values in equation (2.81) for all magnitudes $\in \mathbb{M}_{J}$.

$$
\Lambda\left(\psi>\psi^{*}\right)=\sum_{j=1}^{J} \sum_{k=1}^{n_{G}} P\left(\Psi_{j k}>\psi^{*}\right) \dot{\rho}\left(G X_{k}, G Y_{k}, m_{j}\right) \cdot \Delta A_{k}
$$


Chapter 3

\section{Swarm-Type Sequence}

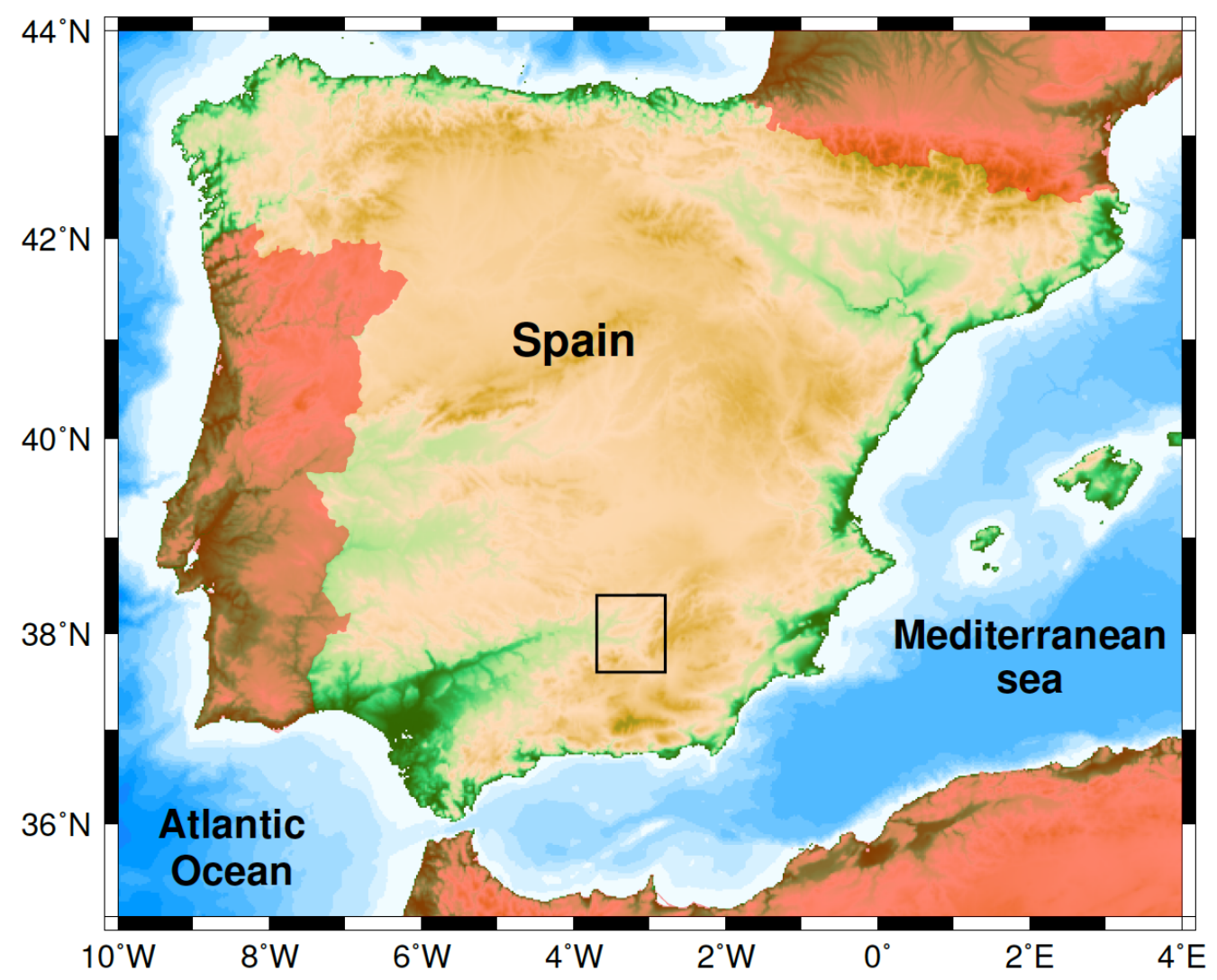

2012-2013 Torreperogil-Sabiote earthquake sequence 



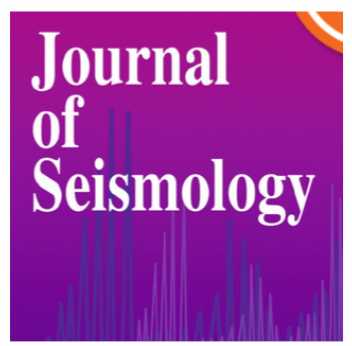

\section{Statistical analysis of the 2012-2013 Torreperogil-Sabiote seismic series, Spain}

\section{Pouye Yazdi (D) - Sebastian Hainzl • \\ Jorge M. Gaspar-Escribano}

\begin{abstract}
The study of earthquake swarms and their characteristics can improve our understanding of the transient processes that provoke seismic crises. The spatio-temporal process of the energy release is often linked with changes of statistical properties, and thus, seismicity parameters can help to reveal the underlying mechanism in time and space domains. Here, we study the Torreperogil-Sabiote 20122013 seismic series (southern Spain), which was relatively long lasting, and it was composed by more than 2000 events. The largest event was a magnitude 3.9 event which occurred on February 5, 2013. It caused slight damages, but it cannot explain the occurrence of the whole seismic crises which was not a typical mainshock-aftershock sequence. To shed some light on this swarm occurrence, we analyze the change of statistical properties during the evolution of the sequence, in particular, related to the magnitude and interevent time distributions. Furthermore, we fit a modified version of the epidemic type aftershock sequence (ETAS) model in order to investigate changes of
\end{abstract}

\begin{abstract}
the background rates and the trigger potential. Our results indicate that the sequence was driven by an aseismic transient stressing rate and that the system passes after the swarm occurrence to a new forcing regime with more typical tectonic characteristics.
\end{abstract}

Keywords Earthquake swarm · Statistical seismology · ETAS · Spain 

Chapter 4

\section{Mainshock-aftershock sequence; crustal faulting(I)}

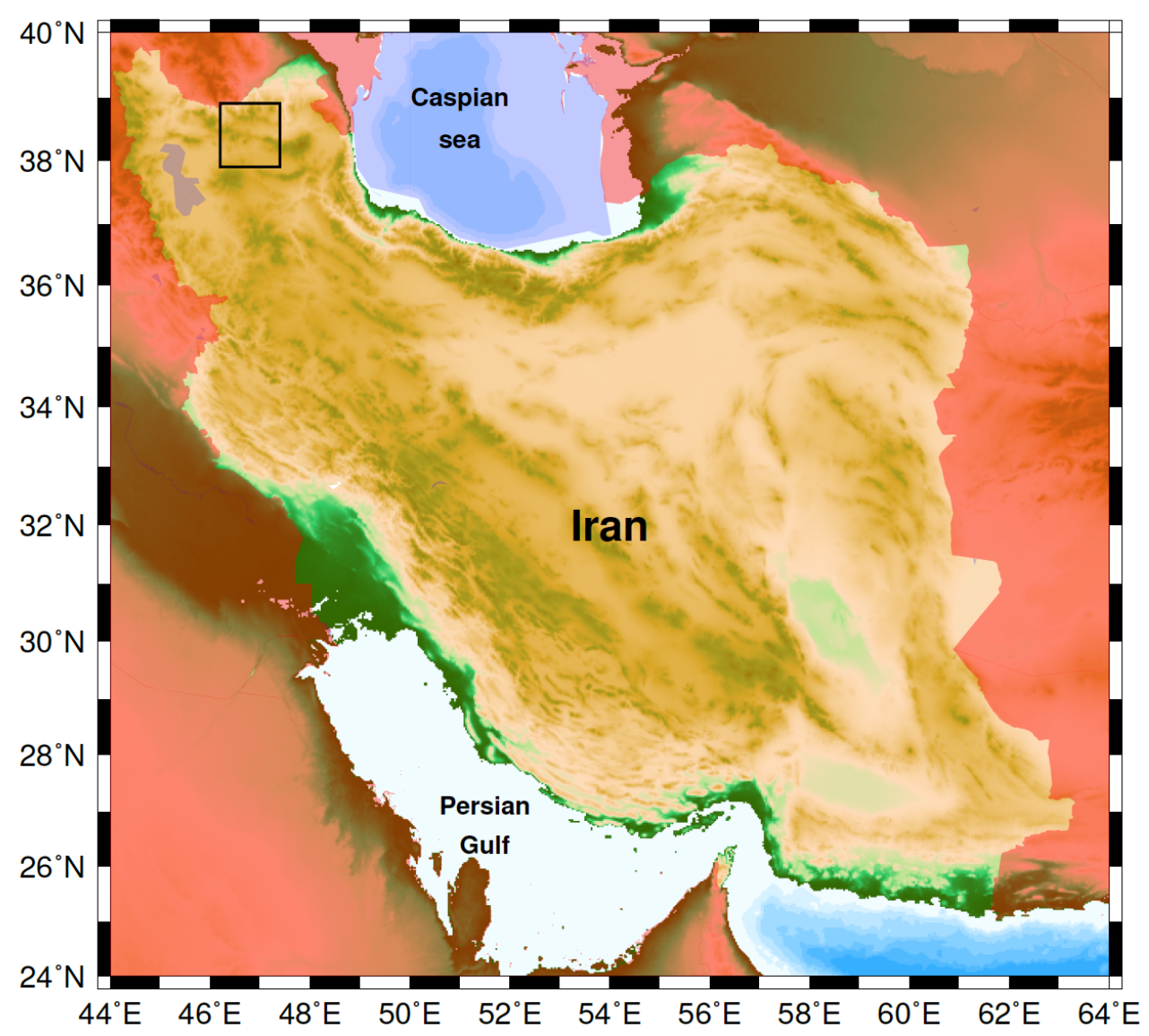

2012 Ahar-Varzeghan earthquake sequence 



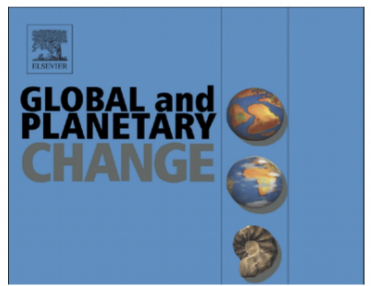

\title{
Analysis of the 2012 Ahar-Varzeghan (Iran) seismic sequence: Insights from statistical and stress transfer modeling
}

\author{
Pouye Yazdi $^{\mathrm{a}, *}$, Miguel Angel Santoyo ${ }^{\mathrm{b}}$, Jorge M. Gaspar-Escribano ${ }^{\mathrm{a}}$
}

${ }^{a}$ Universidad Politécnica de Madrid (UPM), ETSI Topografia, Geodesia y Cartografia, C/Mercator 2, Campus Sur, 28031 Madrid, Spain b Universidad Nacional Autónoma de Mexico (UNAM), Institute of Geophysics in Campus Morelia, 58190 Morelia, Mexico

\section{A R T I C L E I N F O}

\section{Keywords:}

Coulomb stress

Statistical seismology

Background seismicity

Aftershocks

ETAS

Iran

\section{A B S T R A C T}

The 2012 Ahar-Varzeghan (Northwestern Iran) earthquake doublet and its following seismic sequence are analyzed in this paper. First, it is examined the time-varying statistical characteristics of seismic activity since the occurrence of the doublet (two large events with $\mathrm{Mw}=6.4$ and 6.2) that initiated the sequence on 11 August 2012. A power law magnitude-frequency distribution $(1.9 \leq \mathrm{M} \leq 6.4)$ is obtained, with relatively low b-values for the complete series indicating the existence of relatively large magnitudes and high-stress level in the area. The Omori-Utsu model of the aftershock population decay with time shows a moderate decrease in activity rate. An epidemic-type aftershock sequence model that separates background seismicity from triggered aftershocks is then used to describe the temporal evolution of the seismicity during the period following the occurrence of the doublet. Results for the entire series (above cutoff magnitude $\mathrm{Mc}=1.9$ ) indicate a relatively low productivity related to the earthquake-earthquake triggering. Indeed, the majority of these events seems to be generated by underlying transient or aseismic processes, which might be added to the tectonic loading stress. The proportion of aftershock events significantly increases when the analysis is limited to larger events $(M \geq 3.0)$ suggesting that the triggered large aftershocks entail a substantial portion of the energy released.

In order to analyze the spatial distribution of the sequence, new source models are proposed for the two main shocks. For the first shock, the coseismic slip distribution is constrained by the available data on surface ruptures. A Coulomb failure stress transfer model produced by the first event along optimally-oriented planes allows identifying the areas with positive stress loads where the rupture of the subsequent aftershocks may have occurred. The positive $\Delta C F S$ areas are compared for two depth intervals: $3-10 \mathrm{~km}$ and $15-22 \mathrm{~km}$ overlapping over 350 relocated hypocenters, giving arguments supporting the interpretation of $\triangle$ CFS as a main mechanism for aftershock triggering in deeper zones of the upper crust.

Yazdi et al. (2018)

DOI:10.1016/j.gloplacha.2017.12.007 

Chapter 5

\section{Mainshock-aftershock sequence; crustal faulting (II)}

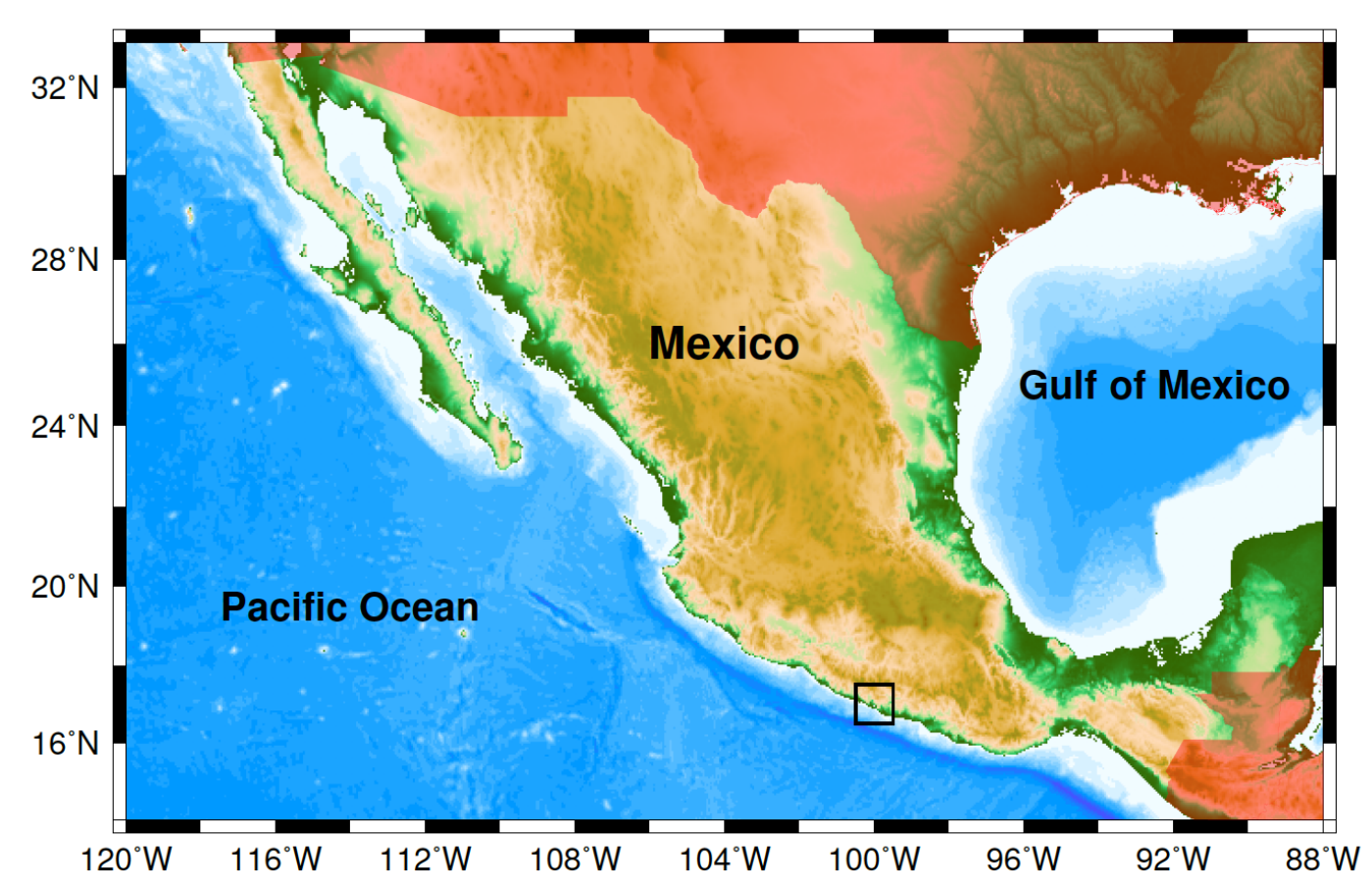

2001 Coyuca earthquake sequence 



\section{The 2001 Coyuca seismic sequence}

In this chapter, I study a crustal earthquake sequence that started on $8^{\text {th }}$ October 2001 with a mainshock of moderate magnitude $M_{\mathrm{w}} 5.8$, with a normal faulting near the town of "Coyuca de Benítez" in southern Guerrero, Mexico. This research involves an application of Coulomb failure stress change ( $\triangle \mathrm{CFS}$ ) computation. The objective is to better understand the influence of static stress accumulation/release processes related to subduction of the Cocos plate beneath the North American (NA) plate in the occurrence of this shallow crustal sequence.

\subsection{Introduction}

The 2001 Coyuca earthquake sequence is located inland and approximately $70 \mathrm{~km}$ away from the Middle American Trench (MAT) in the central coast of the Guerrero. This area is characterized by a very low crustal seismic activity so far (e.g., Iglesias et al., 2004; Pacheco \& Singh, 2010). The mainshock with magnitude $M_{\mathrm{w}} 5.8$ and the following aftershock sequence occurred at shallow depths of about $8-15 \mathrm{~km}$, in the continental crust above the slab interface between the Cocos and the NA tectonic plates (with top of the slab at depths of $\sim 20 \mathrm{~km}$ ). This sequence lasted several months (up to April 2002) and includes over 3000 earthquakes. Zhang et al. (2009) mention a previously unacknowledged Coyuca fault as the possible source for the 2001 Coyuca mainshock. They describe some apparent surface expressions of this fault which suggest its southward dipping that also agrees with the distribution of aftershock series (Iglesias et al., 2004). Table 5.1 shows the location and focal mechanism solution for the Coyuca mainshock.

\begin{tabular}{c|cllccc}
\multicolumn{2}{c}{ Strike } & Dip & Rake & Latitude & Longitude & Depth $(\mathrm{km})$ \\
\hline SSN & $101 / 281$ & $33 / 57$ & $-90 /-90$ & 16.98 & -100.10 & 8 \\
CMT & $104 / 263$ & $53 / 39$ & $-76 /-107$ & 17.32 & -99.89 & 15
\end{tabular}

Table 5.1: Hypocentre location and focal mechanism solutions for $M_{\mathrm{w}} 5.8$ Coyuca mainshock on $8^{\text {th }}$ October 2001 by Servicio Sismólgico Nacional (SSN) of Mexico and Global Centroid Moment Tensor (CMT) catalog.

The upper crustal activity in the central coast of Guerrero can be summarized as composed by occasional earthquakes (with unknown recurrence period) of mainly small magnitude and normal focal mechanism. This area of scant crustal earthquake activity is interestingly located along the Guerrero seismic gap, where the last large earthquake occurred in December 1911 with M7.5. Nishenko \& Singh (1987) determine a recurrence period of 60 to 70 years for earthquakes with M7.7 in this area. This hypothesis implies a "delay" of about 30 years, which implies a higher level of accumulated stress over the subduction interface $(\sim 10 \mathrm{~km}$ below to the mainshock hypocentre) at the time and hypocentral location of the Coyuca sequence. In the following section, the mentioned "delay" and the seismotectonics of this area are explained in more detail. Next, I seek to better understand the influence of subduction processes on the 2001 Coyuca and its aftershock sequence. 


\subsection{Seismotectonics setting}

The seismotectonics of the study area is controlled by the subduction of the Cocos oceanic crust below the NA continental crust. This tectonic process has generated numerous earthquakes with moderate to large magnitudes along the central coast of Mexico during history. In the Guerrero area, the Cocos plate moves northeastward relative to the fixed NA plate (at a rate of 5.5 to $6.5 \mathrm{~cm} . \mathrm{y}^{-1}$; DeMets et al., 2010). Large megathrust earthquakes are principally associated to the highly coupled interplate zones at close distances to the MAT, which have shallow depths (inland or offshore) of less than $25 \mathrm{~km}$ along the coast. Pacheco \& Singh (2010) study the focal mechanism of the earthquakes in Guerrero during a time period of about 13 years (since 1995) and show that for deeper levels (below $25 \mathrm{~km}$ ) the Cocos plate begins to unbend and produces both normal and thrust inslab earthquakes. They believe that at a depth of about $\sim 40(\sim 120$ $\mathrm{km}$ away from MAT) the plate becomes horizontal and involves episodic slow-slip events (see section 5.2.2). The same study finds less intense crustal activity that is characterized by small events with a predominant normal faulting focal mechanism. These events mostly occur after the Coyuca mainshock and close to the epicentral area of this sequence (Pacheco \& Singh, 2010).

\subsubsection{Guerrero seismic gap}

Figure 5.1 shows the ruptured area by subduction earthquakes in central Guerrero presented in studies by UNAM Seismology Group (2015). The absence of large earthquakes since at least 1911 between longitudes $\sim-100^{\circ}$ and $\sim-101^{\circ}$ defines the north-western section of Guerrero seismic gap, where the epicentres of 1899 and 1911 earthquakes have large uncertainty (UNAM Seismology Group, 2015).
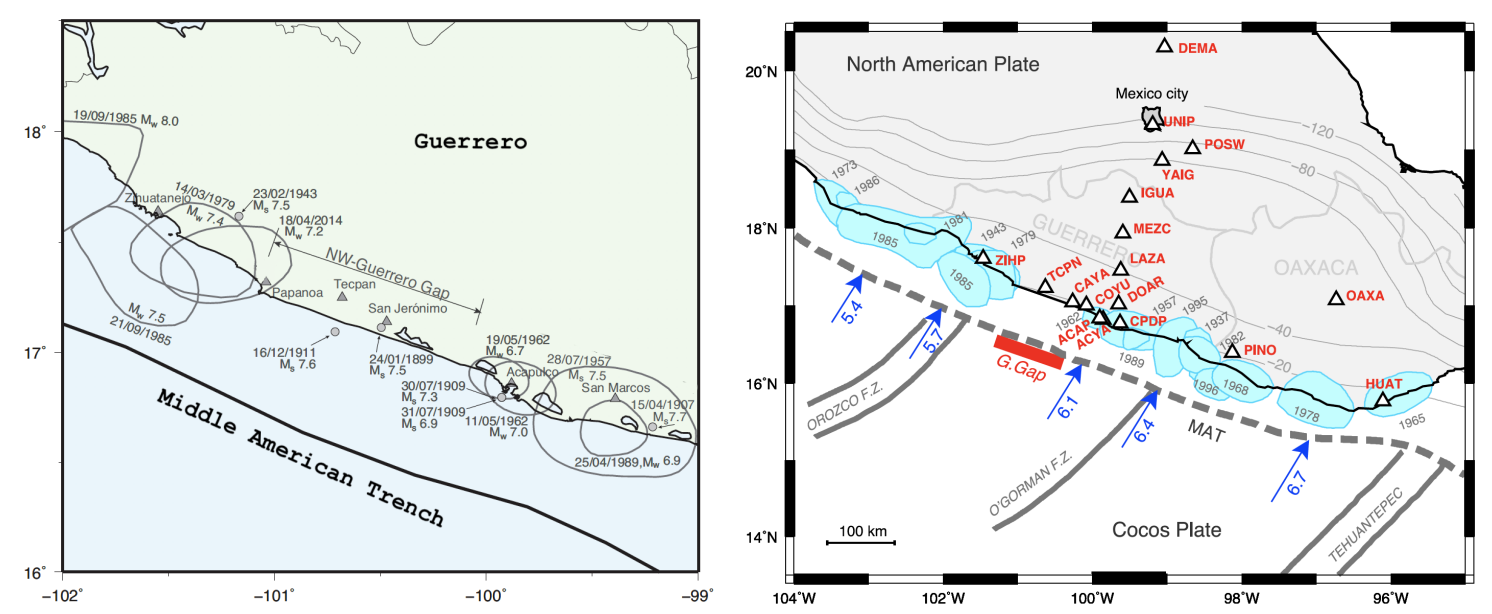

Figure 5.1: Tectonic setting and Cocos plate subducting velocities are shown together with the epicentre and estimated ruptured areas by large megathrust earthquakes near the Guerrero segment in the Mexican subduction. The left caption is adopted from UNAM Seismology Group (2015) and the right caption is from Radiguet et al. (2012).

Graham et al. (2015) and Radiguet et al. (2016) suggest that the Guerrero seismic gap, with a length of about $100 \mathrm{~km}$, is associated with a zone of low coupling in the subduction interface between the Cocos and the NA plates. A low-coupling area presents lower resistance to interface 
slip than a high-coupling under the same stress field. GPS observations on the surface of the NA plate, expose a smaller northward displacement in the Guerrero seismic gap, compared to the rest of Guerrero subduction. This can be due to some downward slip of the Cocos plate at bottom, that does not transmit the entire northward motion to the NA plate. Indeed, the stress accumulation is slower in low-coupling areas and therefore an additional time is required to accumulate the stress necessary to meet the Coulomb criterion (see section 2.3.2), leading to an earthquake rupture. This hypothesis can explain the absence of large earthquakes since a long time, as in Guerrero gap.

\subsubsection{Slow-slip events in Guerrero}

An aseismic slow-slip event (SSE), slides the subduction interface during days to months. These events do not radiate energy in the seismic spectrum. Nowadays, these aseismic phenomena are known thanks to GPS developments. GPS observations show a nearly 4-year periodic northeastward motion in central Guerrero (e.g., Radiguet et al., 2016). The first recognized cycle occurred in mid-1998 and the following cycle began by the end of 2001 reversing its motion with a more rapid velocity towards the southwest in early 2002, associated with the occurrence of an aseismic SSE in 2001-2002 (Franco et al., 2005). SSEs in Guerrero subduction are among the best studied and largest observed in the world. The released energy in a single SSE in this subduction zone maybe in some cases is equivalent to an earthquake with $M_{\mathrm{w}} \sim 7.4-7.6$. Their duration is usually from 6 months up to a year (e.g., Kostoglodov et al., 2003; Franco et al., 2005).

\subsubsection{1-2002 slow-slip event}

The 2001-2002 SSE has been detected by GPS stations around October 2001 in the same area that $1998 \mathrm{SSE}$ and it generated a very large horizontal surface displacement up to $6.6 \mathrm{~cm}$ at GPS station CAYA (Radiguet et al., 2012) in the middle of the Guerrero Gap. The location of GPS stations are shown in Figure 5.1. Kostoglodov et al. (2003) suggest that this SSE lasted for 6 to 7 months (between October 2001 and May 2002) and extended over an area of $\sim 550 \times 250$ $\mathrm{km}^{2}$ with an equivalent moment magnitude of $\sim 7.5$. However, Franco et al. (2005) estimate that the 2001-2002 SSE duration was longer than its apparent active phase. They use long baseline tiltmeters (LBT) that are more sensitive than GPS stations to the onset of SSE. The nearest LBT data to the Coyuca mainshock, close to the ACAP station (with $40 \mathrm{~km}$ distance to Coyuca mainshock), show that the onset of SSE at $40 \mathrm{~km}$ distance, was early October 2001, which is about two months earlier than the onset that GPS data show at ACAP station (i.e., early December 2001). Moreover, the same study reveals a huge anisotropy in the propagation of the slip. Apparently, the SSE started simultaneously at stations CAYA and IGUA, which are located along a perpendicular line to the MAT, and it later arrived to the ACAP station. The SSE near to the Coyuca mainshock (CAYA station) began around mid-August 2001 or early September, and not in October 2001 (as GPS data indicate). Franco et al. (2005) also estimated that the duration of 2001-2002 SSE was not homogeneous over the Guerrero zone and it varied between 9.5 to 11.5 months in the Guerrero gap. Indeed, the estimation of the onset and the duration of SSE are dependent on the selection of the median time of the SSE anomalous displacement toward the 
southwest, yet in this study I consider that 2001-2002 SSE started at least one month prior the 2001 Coyuca sequence, in early September.

\subsection{Data}

Between $8^{\text {th }}$ October 2001 and $31^{\text {st }}$ December 2001, over 3700 aftershocks were recorded by permanent and temporal stations of SSN-National Seismological Service of UNAM ${ }^{1}$ in Mexico. However, this high number of registered events does not construct a complete and homogeneous database because it includes data with missing phases and does not present any event for some days in the middle of the sequence activity period. The temporary stations were installed after 22 days since the start of sequence from $30^{\text {th }}$ October 2001 to $4^{\text {th }}$ December 2001. Moreover, records of some days between this 35-day period are missed and unusable due to damages on the hard discs. Overall, the temporal inhomogeneity in the seismic record precludes any temporal analysis (e.g., Anta-Sánchez, 2017), and hence they are not included in this thesis.

The working database in this study comprises $>1300$ events with hypocentre location. Among them, more than 1100 events can be assigned a magnitude. I attempted to relocate all events of this database using the relocation program hypoDD by Waldhauser \& Ellsworth (2000). Phase data from both temporal and local stations are included up to a radius of $300 \mathrm{~km}$ from the mainshock epicentre. The velocity model by Iglesias et al. (2010) and a velocity rate of $V_{p} / V_{s}=1.73$ are applied. Despite the low restricted conditions used for relocation (minimum of 4 links for the construction of Double-Difference equations), only 235 earthquakes could be relocated (Figure 5.2), and mainly because of the insufficiency in the captured phase data. Setting a low value for the maximum observation per earthquake-pair leads to the involvement of more events in cluster formation and prevents tight clustering. As a result, only two large clusters are identified (Figure 5.8).

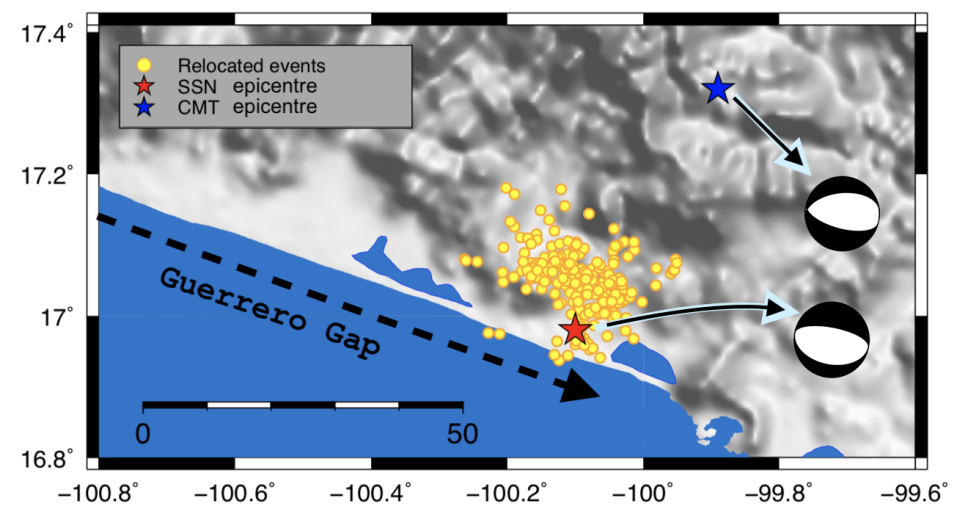

Figure 5.2: Relocated events between $8^{\text {th }}$ October and $31^{\text {st }}$ December 2001. Focal mechanism are from Table 5.1.

In section 5.4, I explain the interplate slip models that I applied for the calculation of $\triangle \mathrm{CFS}$ at the hypocentral location of the Coyuca mainshock and the relocated aftershocks.

\footnotetext{
${ }^{1}$ Universidad Nacional Autonoma de México
} 


\subsection{Subduction interface slip modelling}

This study involves the slip modelling for two consecutive but naturally-different phases of the subduction process at the interplate area between the Cocos and the NA plates. The phaseI is related to an inter-SSE process that starts in mid-1998, and ends with the beginning of the phase-II, that is 2001-2002 SSE. Because of an unclear beginning date associated to the 2001-2002 SSE and its closeness to the 2001 Coyuca sequence (see section 5.2.3), the $\Delta$ CFS calculations are carried out first for phase-I and subsequently for a combination of both (phases-I and phase-II). The applied 3D model for the subduction interface is adopted from Radiguet et al. (2012) (see isolines in the right caption of Figure 5.1) and modified by a downward shift of 1.0 km (Kostoglodov et al., 1996).

\subsubsection{Phase-I: back-slip modelling}

In order to investigate the stress-triggering impact of phase-I on the 2001 Coyuca mainshock, we can estimate the $\triangle \mathrm{CFS}$ associated to the inter-SSE that started prior to this sequence. To model the deformation field because of the Cocos plate movement toward the northeast, I first discretize the subduction interface into patches of $5 \times 5 \mathrm{~km}^{2}$. For simplicity it is assumed that if an interface patch is lowly-coupled or $I \sim 0.0$ (where $I$ is the index of coupling between Cocos and NA plates), then the $0.5 \mathrm{~cm} \cdot \mathrm{mo}^{-1}$ shortening of Cocos plate along the MAT (see right caption in Figure 5.1), entirely transfers as a $0.5 \mathrm{~cm} . \mathrm{mo}^{-1}$ downward motion of the Cocos plate beneath the NA plate, setting a negligible deformation field. In turn, a highly-coupled patch with $I \sim 1.0$ causes a deformation field because it resists the downward slip of the Cocos plate and moves attached to the Cocos plate, toward northwest. This deformation field is equivalent as if this patch experiences a southwestward $0.5 \mathrm{~cm}$ normal slip $\left(\right.$ rake $\left.=-90^{\circ}\right)$. Therefore, I assume that each patch experiences a pure normal slip (rake $\left.=-90^{\circ}\right)$ of size $(0.5 \times I) \mathrm{cm} \cdot \mathrm{mo}^{-1}$. The inter-SSE coupling index is adopted from Radiguet et al. (2016) and smoothed as in Figure 5.3. Because such hypothetical normal slip is in opposite direction of the usual slipping along the subduction interface (thrust slip with rake $=90^{\circ}$ ), this method is called back-slip modelling.

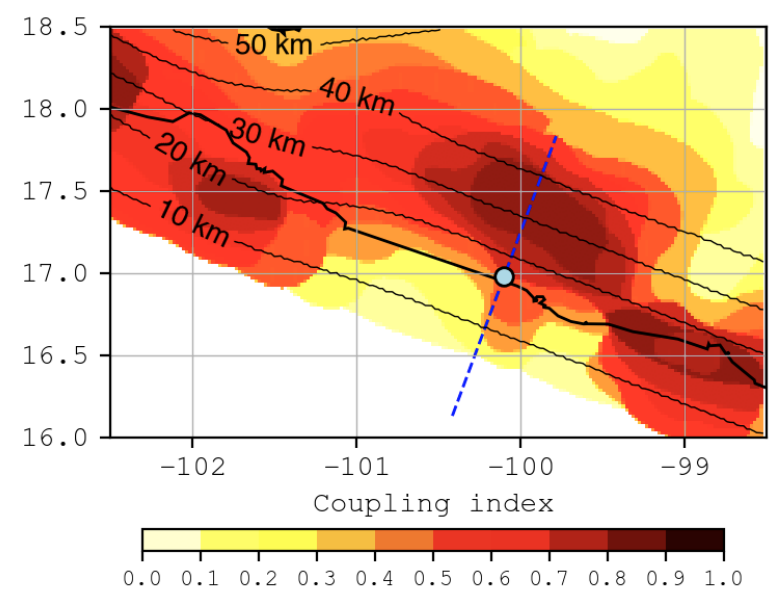

Figure 5.3: Inter-SSE coupling index by Radiguet et al. (2016). The blue circle and the yellow triangle indicate the epicentre of the Coyuca mainshock given by SSN and CMT, respectively. Dashed blue line represents a 200-km long cross section perpendicular to the MAT and passing by these epicentral locations. Think black contours represent the depth to the interface surface. 
The beginning of inter-SSE is set to mid-1998 ( $1^{\text {st }}$ July 1998). The total amount of back-slip during $\mathrm{N}$ months has a size of $\mathrm{N} \times I \times 0.5 \mathrm{~cm}$ over each patch. Therefore, the back-slip distribution maintains the same spatial pattern of the coupling index distribution (Figure 5.3). For the $\triangle \mathrm{CFS}$ calculation of phase-I, the end of inter-SSE period is set on $1^{\text {st }}$ September 2001 (about one month prior to $8^{\text {th }}$ October 2001). Thus, with $\mathrm{N}=38$, I count for the possibility that the 2001-2002 SSE starts before the Coyuca sequence. Alternative possibilities with $\mathrm{N}$ values close to 38 (e.g., the inter-SSE starts one month later than the assumption, the inter-SSE continues until $8^{\text {th }}$ October 2001, and etc.) would change neither the pattern in slip amount (Figure 5.4) nor areas with positive and negative $\triangle$ CFS maps resulting from the back-slip modelling. Such alternative $N$ values (close to 38) will only cause small and uniform variations in the values of slip and hence in the $\triangle \mathrm{CFS}$ distribution, which are not significant to the objective and conclusions in this study.

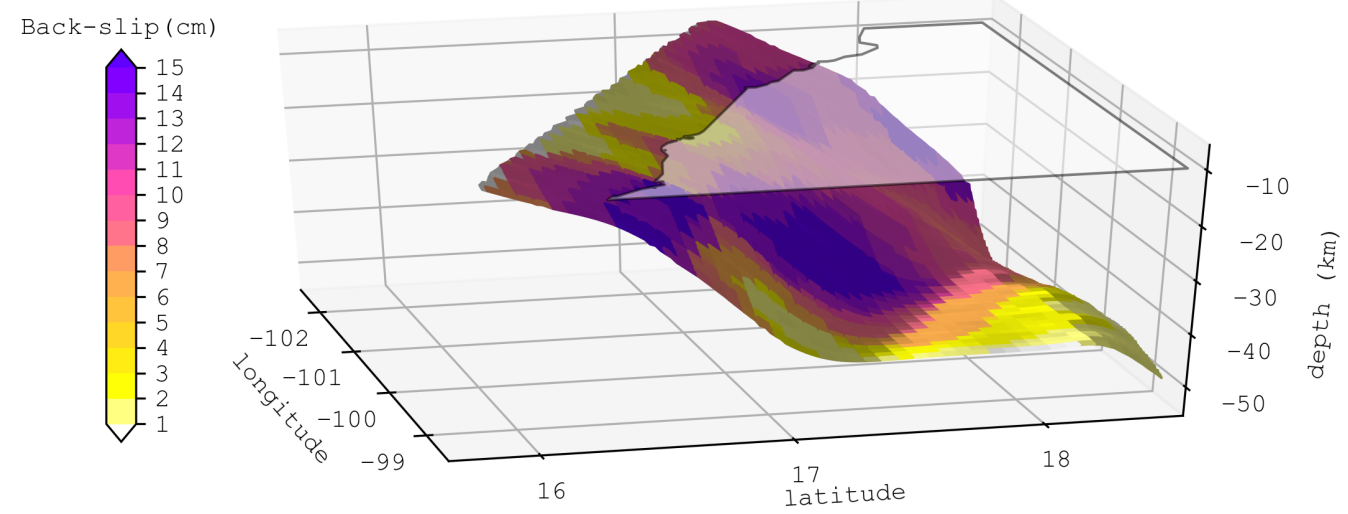

Figure 5.4: Back-slip $\left(\right.$ rake $\left.=-90^{\circ}\right)$ amount during 38 months and applying a constant shortening of 0.5 $\mathrm{cm} . \mathrm{mo}^{-1}$ between longitudes $-98.5^{\circ}$ and $-102.5^{\circ}$. Back-slip $=38 \times 0.5 \times$ coupling index (in Figure 5.3) in $\mathrm{cm}$.

\subsubsection{Phase-II: slow-slip modelling}

Radiguet et al. (2012) studied three SSE phases (2001-2002, 2006 and 2009-2010) in the Guerrero

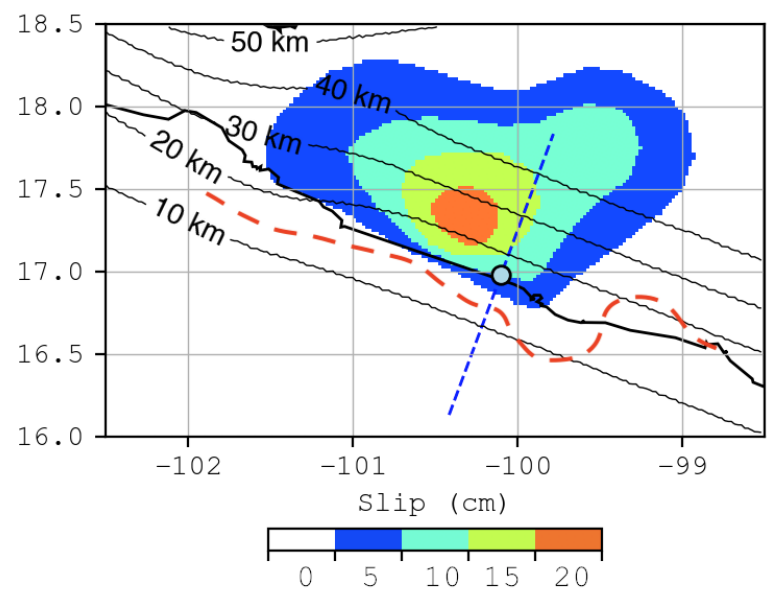

Figure 5.5: A simplified model of the total slip distribution in 2001-2002 SSE, where each zone, represented by different colour, is assigned by its minimum slip value in the original model by Radiguet et al. (2012). The red dashed line shows a total slip of $\sim 0 \mathrm{~cm}$ along the coast as in the original model. Rest of the symbols are as in Figure 5.3. 
subduction zone and modelled the slip velocities with the help of GPS data inversions. They found that the 2001-2002 SSE had an extent of about $<500 \mathrm{~km}$ along the coast and a slip rake of $95^{\circ}$. Figure 5.5 is an adopted and a simplified version of 2001-2002 SSE for modelling the phase-II in this study. Radiguet et al. (2012) estimate a total slip amount of $\sim 20 \mathrm{~cm}$ on the subduction interface in the Guerrero gap. If we assume that the 2001-2002 SSE occurred with a constant rate and with rake angle of $95^{\circ}$ in the subduction interface, and its duration was 10 to 12 months, then one month of slow-slip is equivalent to $10 \%$ to $8.3 \%$ of the total slip in Figure 5.5, respectively. Evidently, applying higher percentages of total slip values yield stronger values of the $\triangle$ CFS in the slow-slip modelling but its pattern will not differ.

\subsection{3 $\Delta$ CFS calculations}

The distribution of $\triangle \mathrm{CFS}$ is calculated for two slip models. Model-I comprises 38 months of back-slip related to the phase-I starting in mid-1998 and ending by September 2001. Model-II is made of $10 \%$ of total slow-slip in phase-II to incorporate the possibility that the 2001-2002 SSE initiates before the Coyuca mainshock, in early September (see section 5.2.3). Then, the $\triangle \mathrm{CFS}$ resulting from both models, are added-up to show the overall triggering influence of 38 months of back-slip in phase-I plus $10 \%$ of slow-slip in phase-II. I apply two apparent coefficients of friction friction $\mu^{*}=0.4$ and $\mu^{*}=0.6$, standing for less and more dryness conditions, respectively. However, all the $\triangle$ CFS figures I provide in this chapter are calculated with $\mu^{*}=0.4$ and rigidity modulus $\mathrm{G}=38 \mathrm{GPa}$. The influence of $\mu^{*}=0.6$ is not significant for the results, and hence not shown in all the figures. The epicentre by CMT is far away $(\sim 30 \mathrm{~km})$ from the aftershock grouping (Figure 5.2), thus I only calculate the $\triangle \mathrm{CFS}$ for the southward nodal plane of the focal mechanism solutions by SSN in Table 5.1.

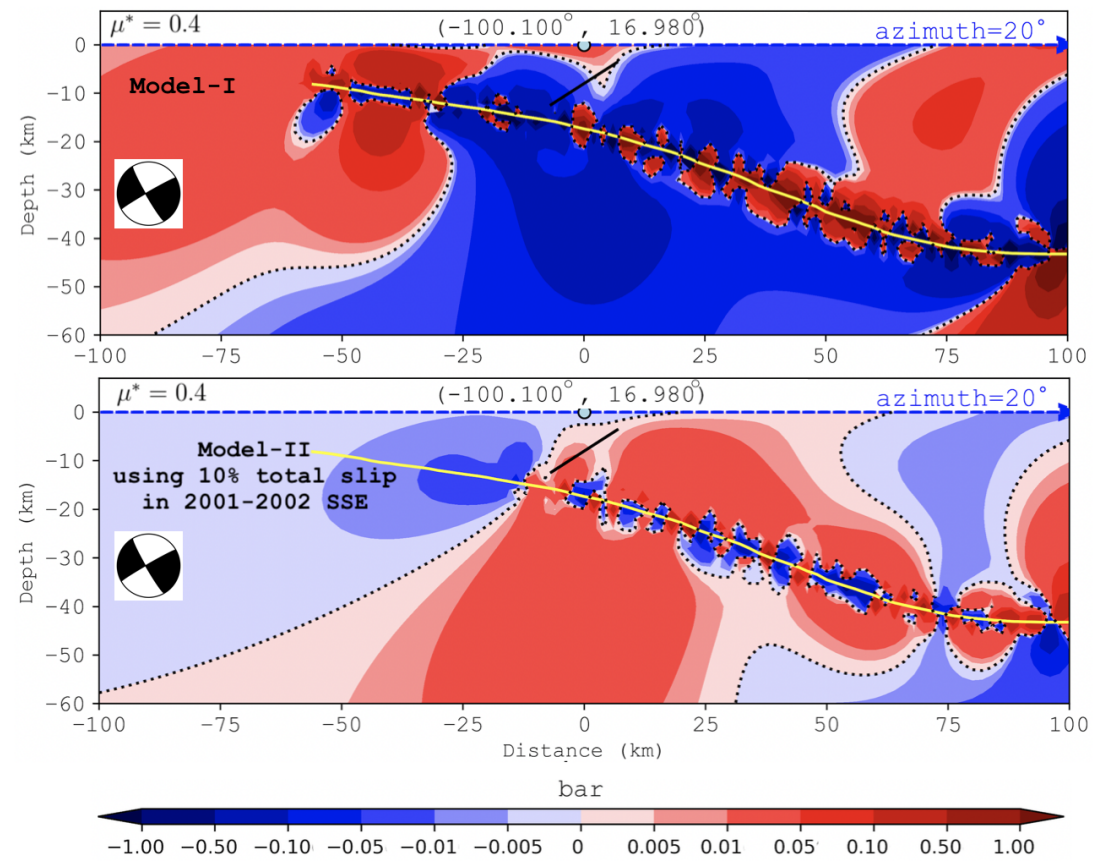

Figure 5.6: The $\triangle \mathrm{CFS}$ for the southward nodal plane by SSN, on the cross section in Figure 5.3 and applying model-I (up), and $10 \%$ of total slip in model-II (down). Dashed black line indicates $\triangle \mathrm{CFS}=0$. 
Figure 5.6 shows the results for model-I and model-II and Figure 5.7 shows their sum together with the re-located aftershocks. To indicate the approximated cross section of rupture plane with $\triangle$ CFS calculation profiles, the proposed rupture plane by Iglesias et al. (2004) is added to the $\triangle \mathrm{CFS}$ maps. This rupture plane has a length of $\mathcal{L}=12 \mathrm{~km}$ and a width of $\mathcal{W}=16 \mathrm{~km}$ and I set the mainshock hypocentre at the centre of this rectangular geometry. As it is shown in Figure 5.6, the back-slip modelling of inter-SSE period since mid-1998 (model-I) does not show an evident triggering impact due to the tectonic stress accumulation in this period. In turn, a $10 \%$ of total slow-slip during 2001-2002 SSE (model-II) exhibits static stress change with a maximum of $\sim+0.01$ bars at the hypocentral location of the mainshock. However, Figure 5.7 shows that the triggering influence of this early stage of 2001-2002 SSE, is too small to change the overall distribution of $\triangle \mathrm{CFS}$ with respect to the result of model-I. At a depth of $8 \mathrm{~km}$, which is given as the hypocentral depth (Iglesias et al., 2004, SSN), the $\Delta$ CFS is almost zero. At a shallower depth of $\sim 5 \mathrm{~km}$, it becomes positive, though it is small $(<+0.01$ bars $)$, and at a higher depth close to $10 \mathrm{~km}$ it is $\sim-0.1$ bars.

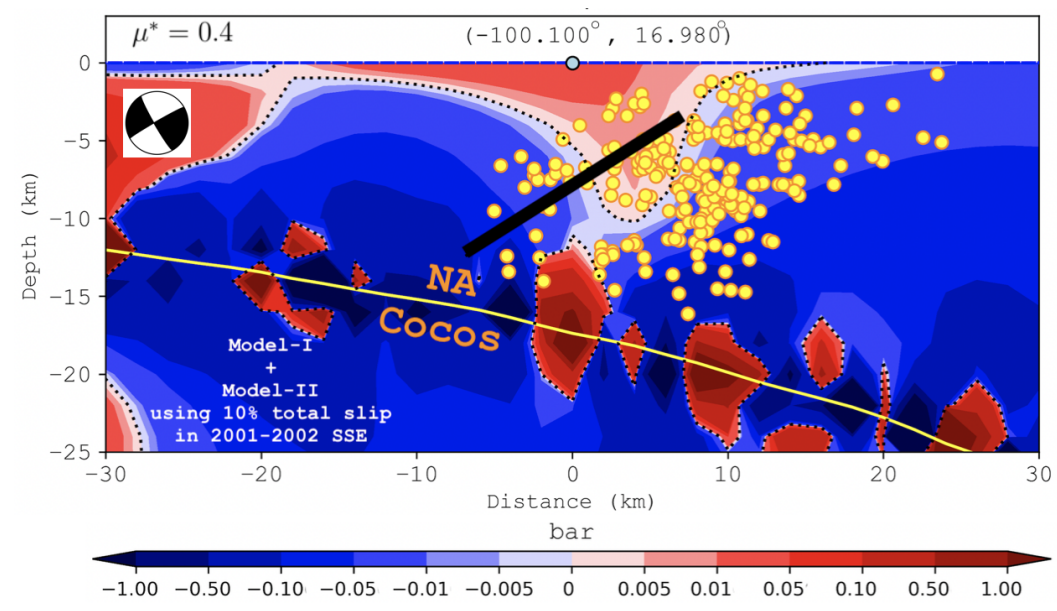

Figure 5.7: The $\triangle$ CFS for the southward nodal plane by SSN, on the cross section in Figure 5.3 (here is zoomed) and applying the summation of slip model in model-I and $10 \%$ of total slip in model-II. Yellow dots show the relocated events in Figure 5.2. Dashed black line indicates $\triangle \mathrm{CFS}=0$.

The re-location of the aftershock sequence leads to two main clusters in space dimension: clusterI and cluster-II with 99 and 103 events, respectively. As it is mentioned in section 5.3, the temporal discontinuousness of earthquake recording during this sequence prevents identifying their clustering behaviour in time. With respect to these two clusters, two 100-km long vertical cross sections (or profiles) perpendicular and parallel to the MAT are considered for $\triangle \mathrm{CFS}$ estimation applying the model-II (Figure 5.9). The cross section parallel to the MAT is passes through the cluster-I, and the cross section perpendicular to the MAT is passed through the cluster-II. These new profiles cross at coordinates $100.05^{\circ} \mathrm{W}$ and $17.05^{\circ} \mathrm{N}$. 

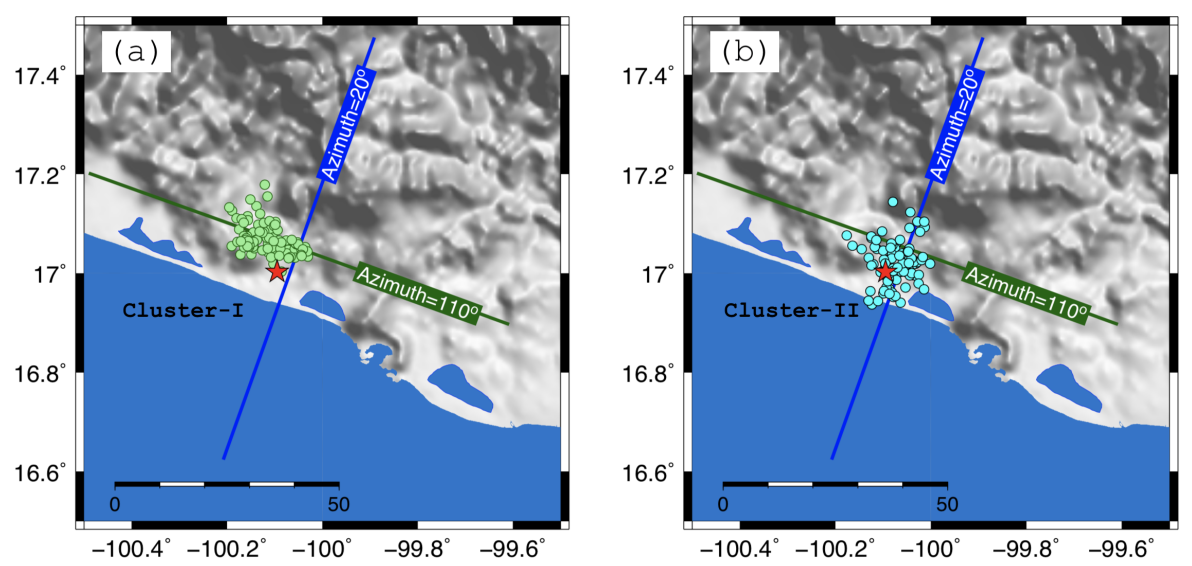

Figure 5.8: The two clusters after re-location of aftershocks. Cluster-I has 99 earthquakes (a) and clusterII has 103 earthquakes (b). Both clusters have a southwestward dipping. Blue and green lines represent $100-\mathrm{km}$ long cross sections perpendicular and parallel to the MAT, respectively.

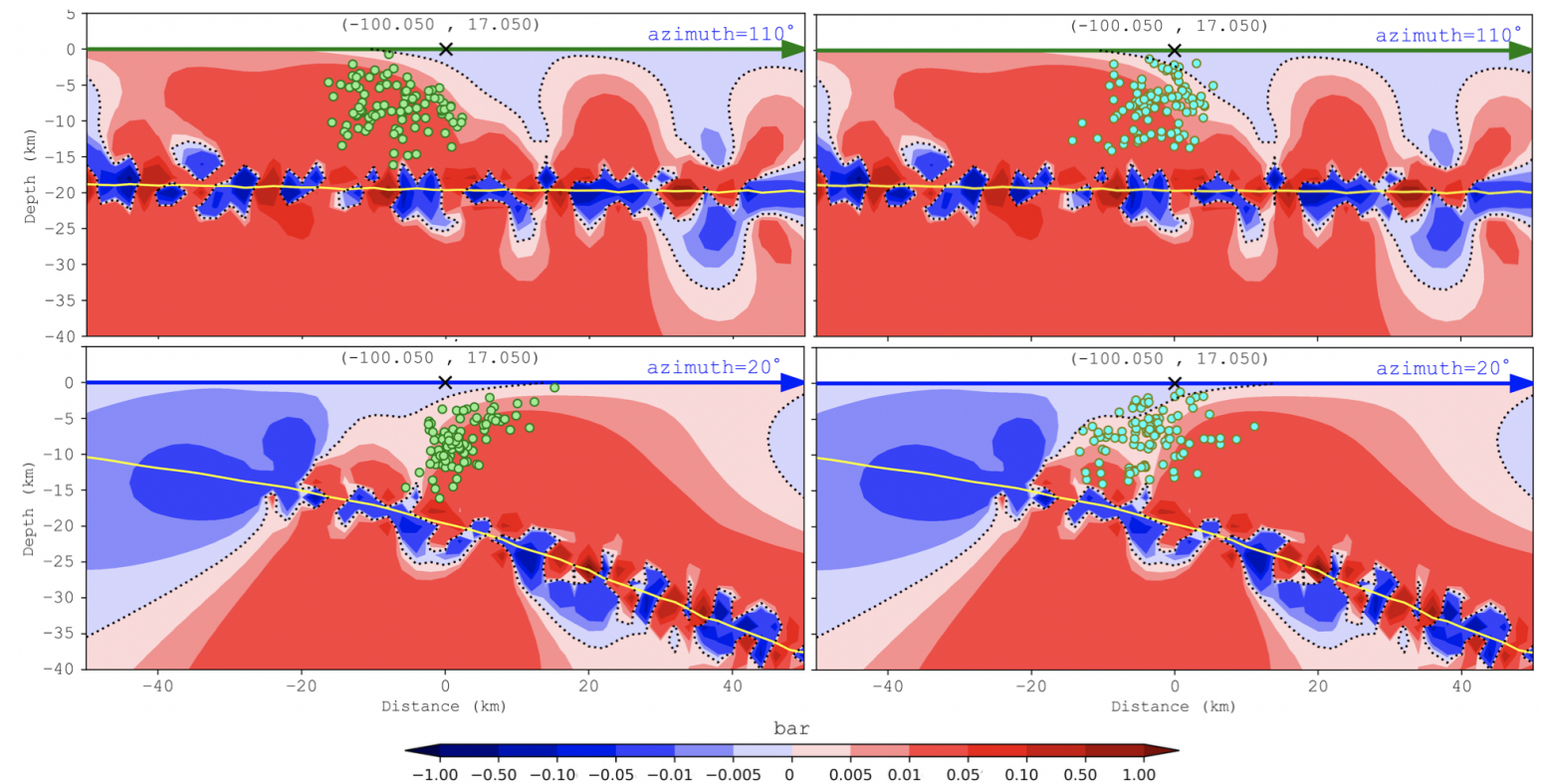

Figure 5.9: Distribution of $\Delta$ CFS for model-II with $10 \%$ of slow-slip in 2001-2002 with rake of $95^{\circ}$, and for focal mechanism $101^{\circ} / 33^{\circ} /-90^{\circ}$ (SSN), on the two cross sections in Figure 5.8. The hypocentral location of cluster-I (left) and cluster-II (right) are projected on both profiles.

I did carry out the $\triangle$ CFS computation for both nodal planes by SSN and along the perpendicular profiles of Figure 5.8. The result is consistent for both nodal planes, thus I only present the $\triangle \mathrm{CFS}$ result for the southward mechanism $\left(101^{\circ} / 33^{\circ} /-90^{\circ}\right)$ in Figure 5.9. This figure confirms the favouring influence of slow-slip beginning on the occurrence of Coyuca aftershock sequence as these events have same focal mechanism as the mainshock. Moreover, the horizontal projection of hypocentres in the cluster-I, conforms very well with the spatial pattern of positive $\triangle \mathrm{CFS}$, in both profiles (left captions in Figure 5.9). 


\subsection{Discussion and conclusions}

The objective of this study is to determine the possible triggering influence of subduction processes on the occurrence of the 2001 Coyuca shallow crustal earthquake sequence with a mainshock of $M_{\mathrm{w}} 5.8$ and normal mechanism. The applied physics-based approach involves the computation of changes in the static stress due to two different aseismic activities: (1) tectonic loading, and (2) slow-slip event in the Mexican subduction zone.

The $\triangle$ CFS resulting form tectonic stress accumulation since mid-1998, over the subduction interface of the Cocos and the NA plates, does not reveal an evident aseismic triggering influence on the 2001 Coyuca mainshock. Actually, the mainshock rupture plane by Iglesias et al. (2004) lies in the area of transition between negative and small positive $\triangle$ CFS changes (lower and upper parts of the rupture plane, respectively). In turn, the beginning of a slow-slip event, only few weeks before this mainshock, discloses a static stress triggering impact for normal rupturing at the shallow crust near the Coyuca sequence. Moreover, the positively charged areas during these few weeks, highly coincide with the hypocentral distribution of re-located aftershocks. Nevertheless, this resulting stress distribution does not make a significant change when its effect is added to the effect of back-slip modelling.

A hypothesis would be that the Coyuca mainshock is triggered following the start of 2001-2002 SSE, where a rapid change occurs in the NA crust northeastward motion to the southwest, and it increases the Coulomb stress at shallower depths and ruptures the Coyuca fault. This interpretation requires to assume that the pre-existing southward normal fault (i.e., Coyuca fault) was very close to rupture just before the previous inter-SSE began in mid-1998. Otherwise, as it is shown in lower caption of Figure 5.6, the $\triangle \mathrm{CFS}$ is too small to launch a casual extensional mechanism in an area of a generally tensional regime.

With respect to the given hypothesis, the inter-SSE period alleviates the stress accumulation over the Coyuca fault, and by the beginning of an upward (to southwest) slip in the NA, the Coyuca fault meets the Coulomb criterion at the shallow depth and ruptures. It is worth remarking that the slip model of the Coyuca mainshock by Iglesias et al. (2004) shows that the highest slip or the centroid has a depth of $5 \mathrm{~km}$, which supports this hypothesis. The continuation of the aftershock sequence and their hypocentral distribution is also consistent with the completion of SSE period. The exact date of inversion in the direction of the NA's movement, can determine a better estimation of $\triangle \mathrm{CFS}$ values. If we assume that a $40 \%$ of the SSE took place by the end of 2001 , then the $\triangle$ CFS because of model-I and model-II at the end of 2001 would be as in Figure 5.10. The two captions show a comparison between results for less saturated conditions with $\mu^{*}=0.6$. This figure displays that the spatial expansion of the positively charged areas coincides with the hypocentral distribution of aftershocks. This result is relevant with the given hypothesis. 


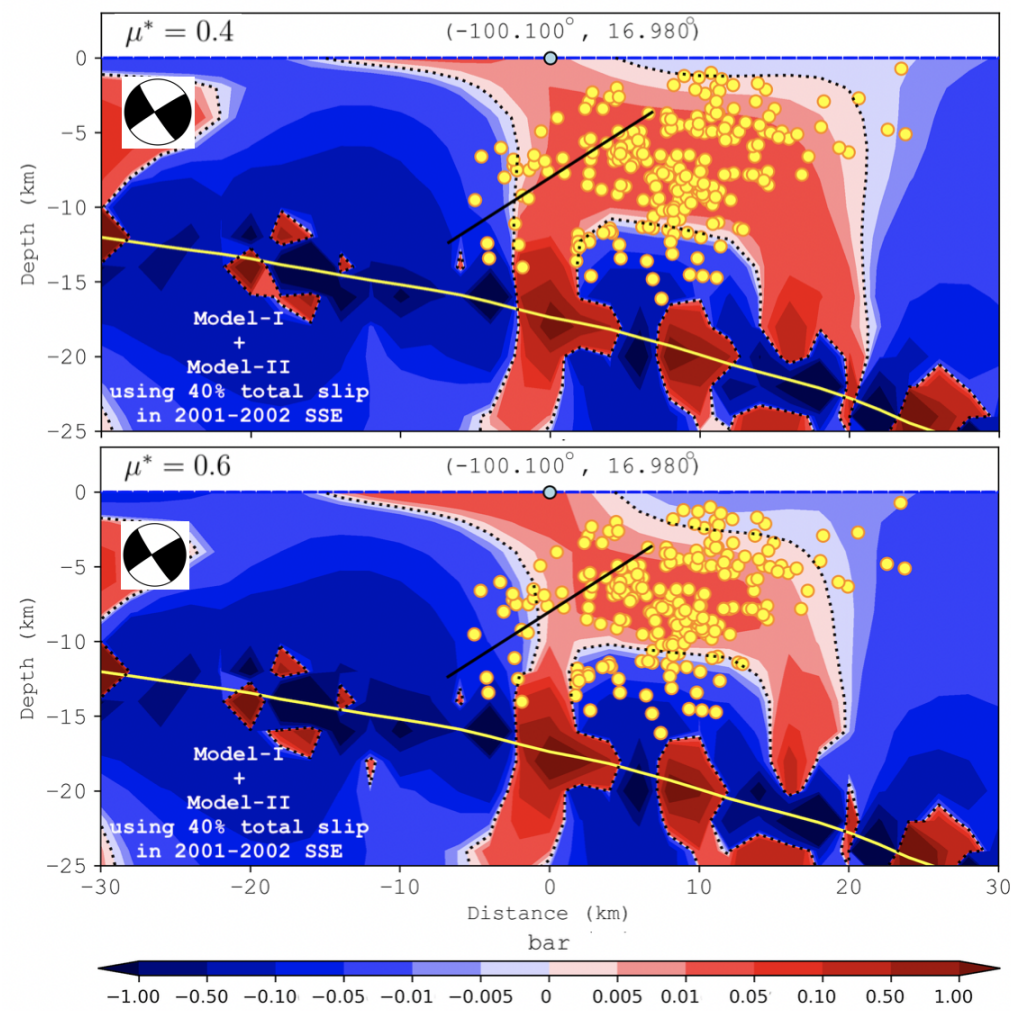

Figure 5.10: The $\triangle \mathrm{CFS}$ for southward nodal plane by SSN, on the cross section in Figure 5.3 (here is zoomed) and applying the summation of slip model in model-I and $40 \%$ of total slip in model-II. Rest of the symbols are as in Figure 5.7.

Overall, the results of this study significantly rely on the distribution of the applied coupling index, slow-slip model and the 3D geometry of the subduction interface. To show that, I repeat the calculation shown in the upper caption of Figure 5.10 for some parallel cross sections at 5 , 10,15 and $20 \mathrm{~km}$ to the east and to the west of the initial profile.

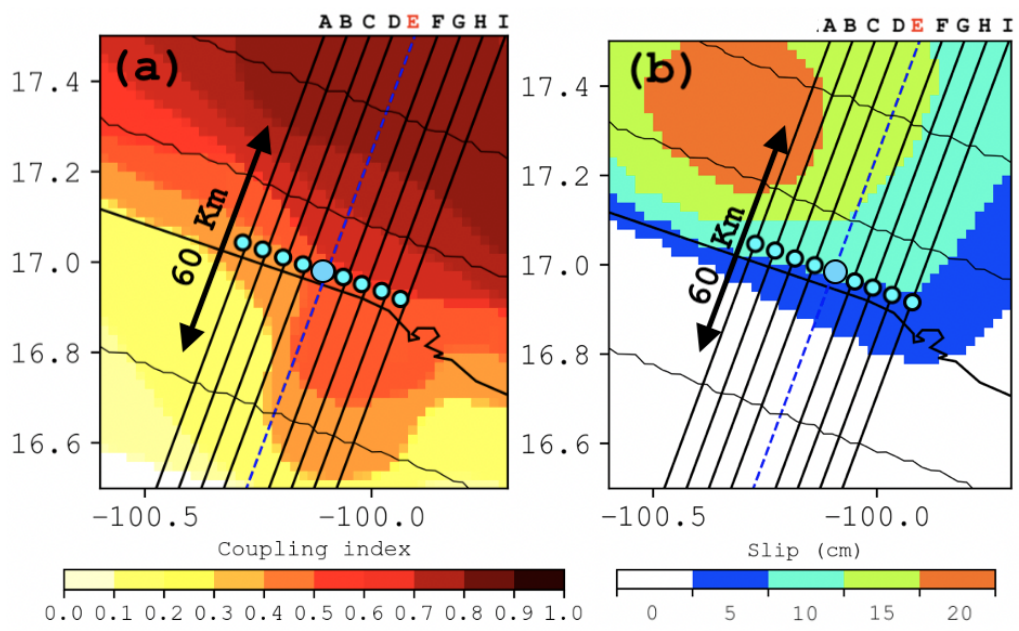

Figure 5.11: A zoomed caption of Figure 5.3 (a), and Figure 5.5 (b), for a better resolution in showing the west-side cross sections (profiles A, B, C and D) and the east-side cross sections (profiles I, H, G and F), with horizontal distances of $20,15,10$ and $5 \mathrm{~km}$, respectively, to the main cross section (the same as in Figures 5.3 and 5.5) or profile E. Blue dots indicate the upper centre of each profile. 
The considered profiles in Figure 5.11 have an azimuth of $20^{\circ}$ and are placed with $5 \mathrm{~km}$ separation along an orientation of $110^{\circ}$ (along the MAT).

The $\triangle$ CFS for Coyuca mainshock southward nodal plane (as given by SSN) is estimated for the cross sections in Figure 5.11. Figure 5.12 shows the result within $\pm 30 \mathrm{~km}$ from the upper centre of each profile and down to a depth of $25 \mathrm{~km}$. The results illustrate considerable gradual changes in the Coulomb stress pattern along the azimuth of $110^{\circ}$.

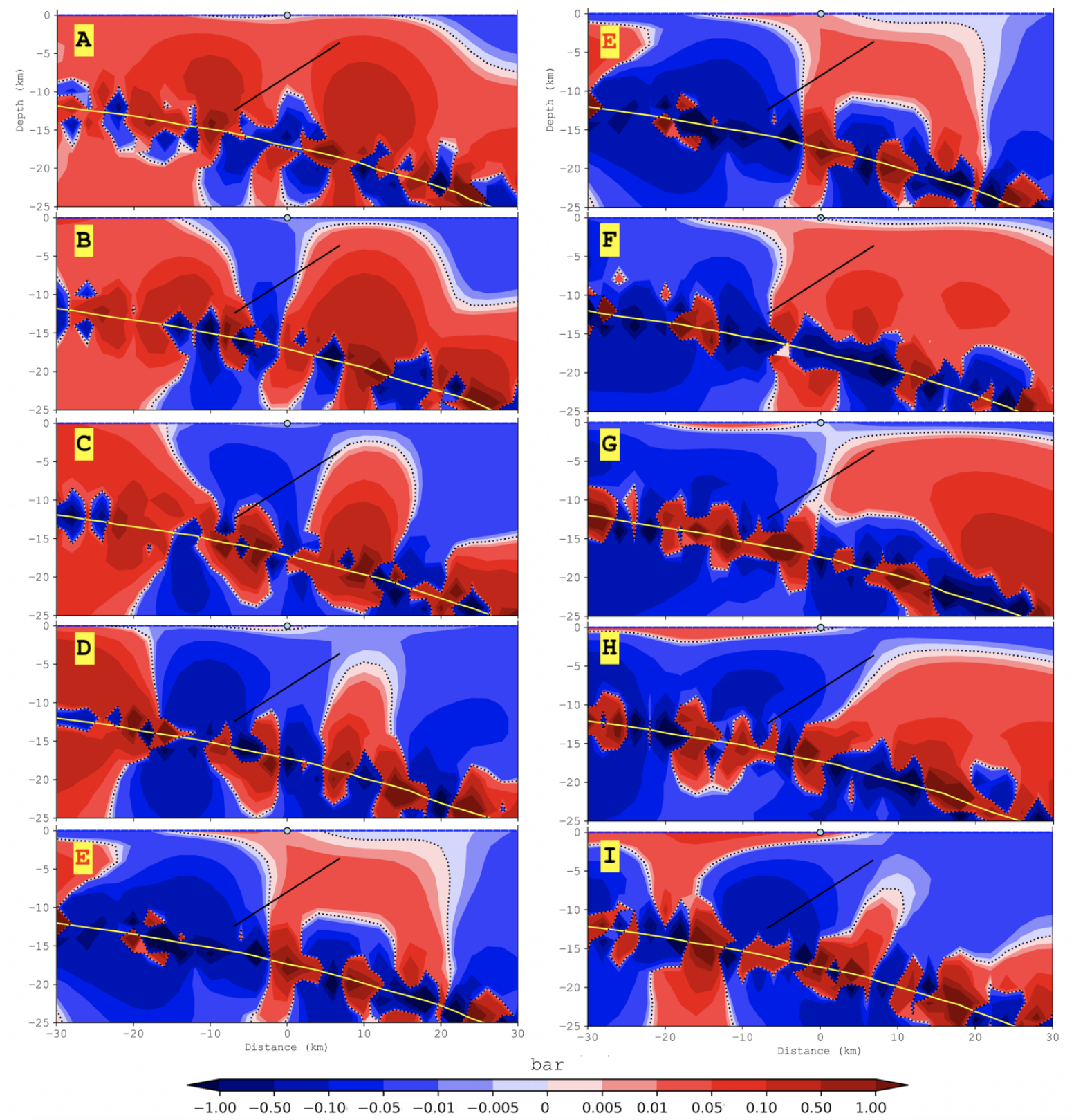

Figure 5.12: The $\triangle \mathrm{CFS}$ for southward nodal plane by SSN, on the cross sections in Figure 5.11 and within the zoomed window as in Figure 5.10. The applied slip models is the summation of slip in model-I and $40 \%$ of total slip in model-II. Dashed black line indicates $\Delta \mathrm{CFS}=0$.

Profiles A, B , C and D run across the areas where the coupling index distribution is not significantly variant, but the $\triangle \mathrm{CFS}$ distributions in these profiles show evident changes that are likely due to the reduction of slow-slip amount at the northern part of the profiles (from $20 \mathrm{~cm}$ to 
$15 \mathrm{~cm}$ in its total value). The main cross section (profile E) coincides with higher coupling index in the offshore, which results the expansion of negative $\triangle \mathrm{CFS}$ values toward the south. The coupling index and slow slip distribution for profiles F, G, H and I present slight changes that are consistent with the $\triangle \mathrm{CFS}$ maps for profiles F, G and H. However, the slight southward progress in the offshore slow-slip ( $5 \mathrm{~cm}$ in its total value) in the eastern cross sections (profiles $\mathrm{G}, \mathrm{H}$ and I) could be responsible for the expansion of positive $\triangle$ CFS at shallow depths. The geometry of the subduction interface and the applied patches for the $\triangle$ CFS estimation are rather constant in this area. Therefore, the observed changes in the $\triangle \mathrm{CFS}$ distribution in Figure 5.12 are related to the variation in the used slip distribution, which is composed by back-slip in Model-I and a percentage of slow-slip in Model-II.

In fact, the achieved $\triangle$ CFS maps in Figure 5.12 highlight the importance of uncertainties in the input data for the slip modelling. The evident influence of slow-slip contribution in the $\triangle \mathrm{CFS}$ along cross sections with alike back-slip distribution remarks the sensibility of the triggering impact to gradients of this contribution. Indeed, the introduced hypothesis is conceivable, yet should not be extrapolated to the shallow crust over the Guerrero gap without evident information about the presence of active normal faulting. 

Chapter 6

\section{Subduction sequence; large thrust earthquake}

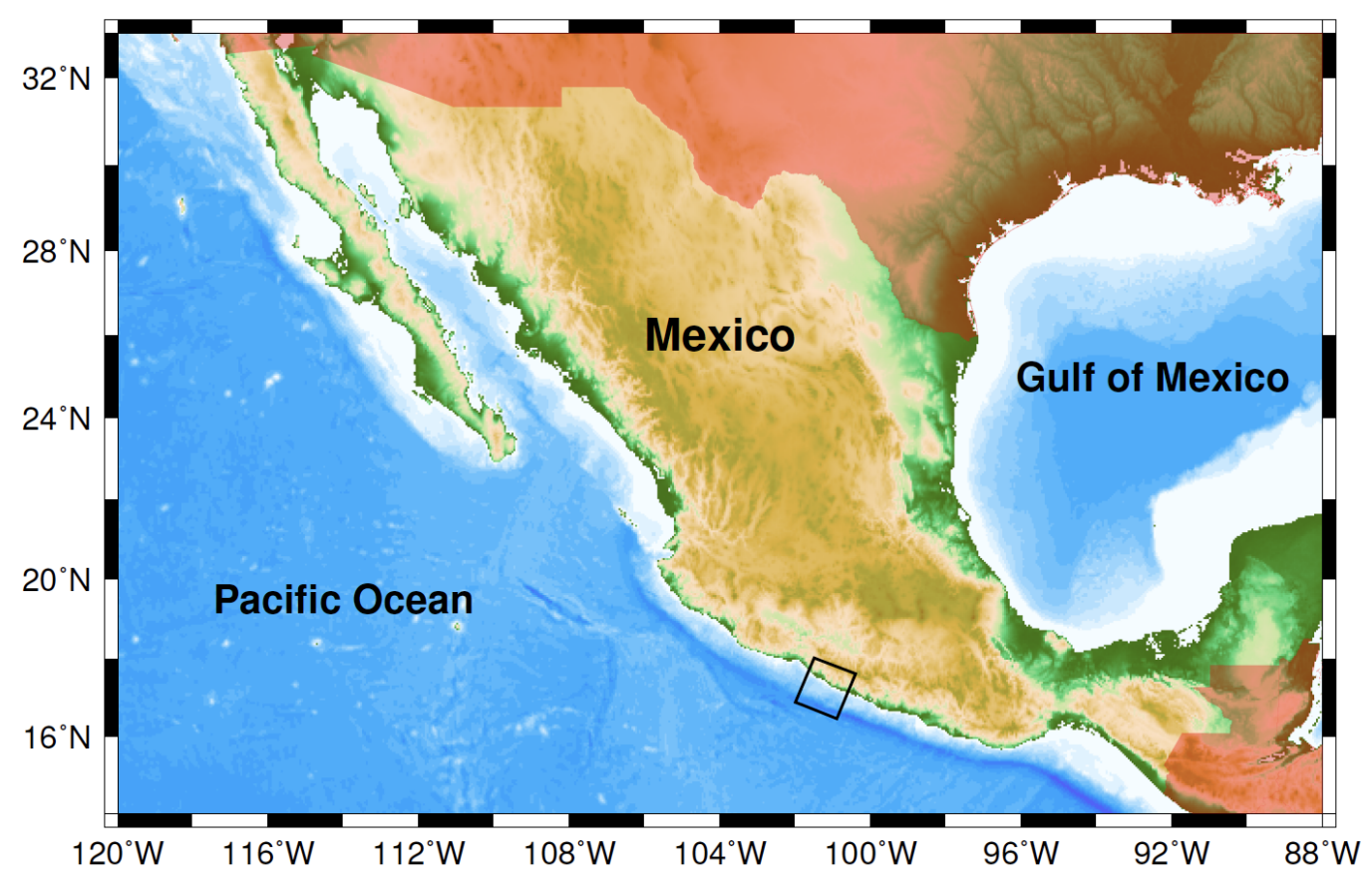

2014 Papanoa earthquake sequence 



\title{
Analysis of the $2014 M_{\mathrm{w}}$ 7.3 Papanoa (Mexico) Earthquake: Implications for Seismic Hazard Assessment
}

by Pouye Yazdi, Jorge M. Gaspar-Escribano, Miguel A. Santoyo, and Alejandra Staller

\begin{abstract}
We analyze the 2014 Papanoa seismic series, located in the Guerrero, Mexico, subduction interface. First, Coulomb failure stress changes because of the mainshock, and the two largest aftershocks are calculated along the interface. The location of aftershock epicenters largely coincides with areas showing positive stress changes. In addition, epidemic-type aftershock sequence modeling of the series indicates aftershock productivity was not especially large and suggests an increase of background activity rates after the occurrence of the 2014 Papanoa series. This information is used to infer the magnitudedependent, smoothed densities of background rates for the study area. Ground-motion exceedance rates because of interface sources are computed considering different seismicity rate densities and applying the declustering approach. Results show a slight increase of expected accelerations for different exceedance probabilities.
\end{abstract}





\section{Chapter 7}

\section{Summary and Conclusions}

The four earthquake sequences studied in this dissertation reflect the result of my survey through the analysis of earthquake sequences in some distinct geographical zones with different seismotectonics. These analyses share similar approaches for investigating the addressed aspects of the earthquake sequences.

The statistical approach is applied to analyse the earthquake sequences assuming that they are outcomes of a random point process that generates earthquakes (points) with random magnitude, time and location. This approach searches for the laws that best rule the earthquake process and tends to identify the probability distributions of earthquake parameters by performing and testing the stochastic models in finite time and space windows. The extrapolation of the modelled activity rates beyond this windows does not contemplate any changes along the process of earthquake generation. Nevertheless, this approach can be insightful in analysing the causality of the earthquake sequences, which is an essential input towards earthquake forecasting effort.

For all the studied sequences, I initially take the distribution of earthquake magnitudes as the main key for classifying the earthquake sequences into mainshock-aftershock or swarm types. In chapters 3, 4 and 6, I perform a magnitude-frequency analysis using the Gutenberg-Richter law and I use the Epidemic Type Aftershock Sequence (ETAS) model to characterize each sequence. This model considers that the earthquakes in a sequence epidemically induce a new cascade of aftershocks. Thus, I inquire its applicability and performance for understanding the contribution of aseismic and seismic forcings in the generation of the earthquake sequences. Figure 7.1 gathers the ETAS model prediction for the accumulative number of events versus time in the three mentioned chapters with a very close adjustment to the observations, showing that the ETAS model is highly capable of predicting the observed activities.

A brief conclusion about what I have achieved through statistical analyses of the introduced sequences in chapters 3, 4 and 6 is listed below: 

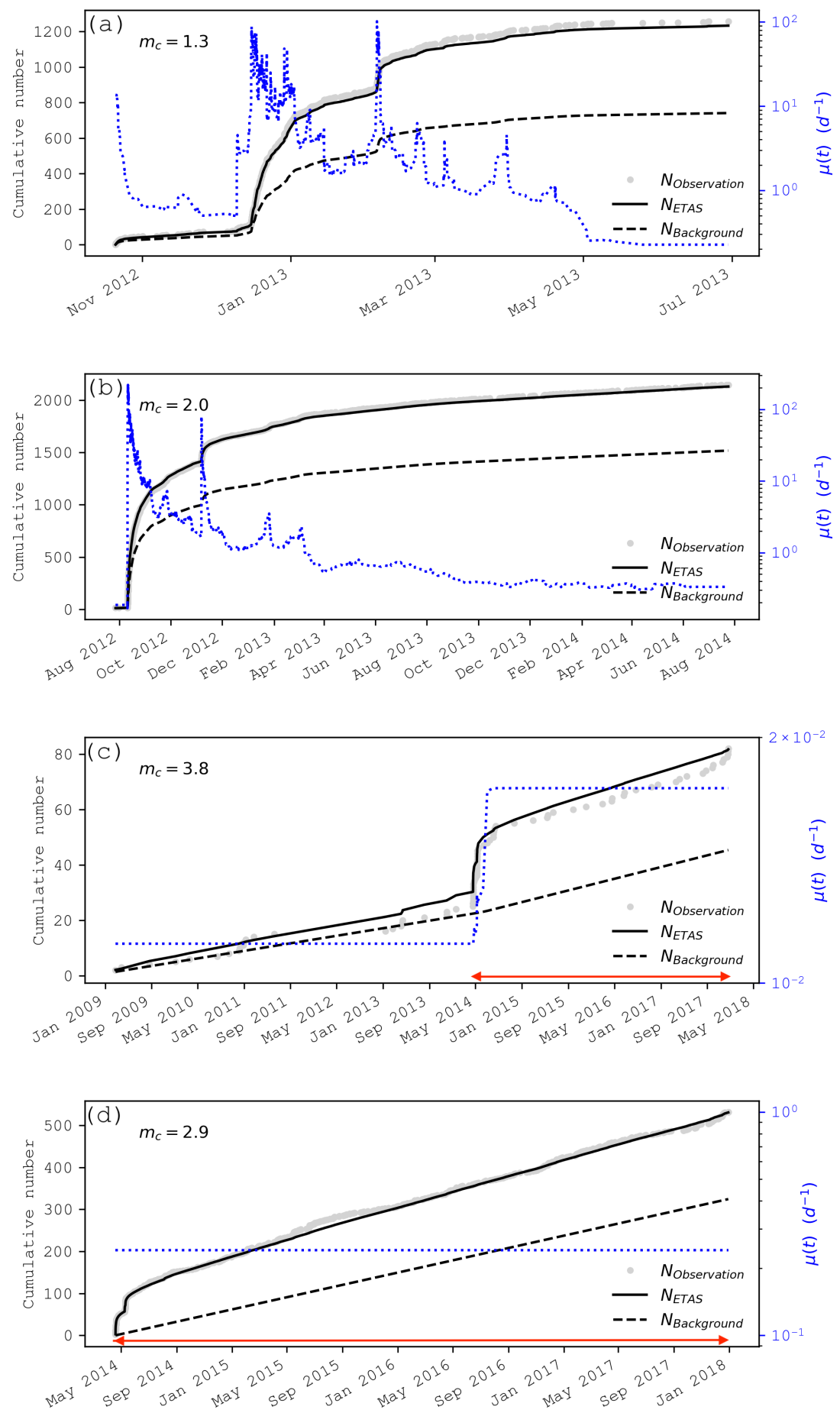

Figure 7.1: Results of the ETAS modelling in the time domain for the earthquake sequences of 2012-2013 Torreperogil-Sabiote: main activity phase (a), 2012 Ahar-Varzeghan (b), and 2014 Papanoa (c and d).

In Yazdi et al. (2017), we model the activity rates related to the swarm series of 2012-2013 Torreperogil-Sabiote, during the pre-, main- and post-activity phases of this long-lasting, highly-clustered sequence (chapter 3). We examine the temporal variations in the background activity rate $\mu(t)$ and we identify the aseismic forcing as the leading process for the earthquake generation in all three phases. The temporal 
ETAS modelling shows that during the main-activity phase, $\mu(t)$ significantly rises from an average of $\sim 0.1$ to $\sim 2.9$ events per day. Consequently, the aftershock contribution also rises from $\sim 20 \%$ to $\sim 40 \%$ in this phase. On the other hand, the productivity parameter $\alpha=0.975$ during this phase (with $m_{c}=1.3$ ), is smaller than its typical values $(\gtrsim 1.5)$ for mainshock-aftershock sequences (e.g., Hainzl \& Ogata, 2005; Hainzl et al., 2013) and agrees with a swarm-type activity. The low contribution of earthquake-earthquake triggering does not support the tectonic loading (source of the tectonic earthquakes that are usually followed by numerous aftershocks) as the only aseismic source in the generation of background events. We suggest that the observed rise in background activity might be influenced by some additional forcing, apart from the tectonic loading and internal stress interactions. The GR modelling shows a significant decrease of $b$-value from 1.5 to 1.1 in the main-activity phase, which is due to the occurrence of bigger events (including the largest earthquake of the sequence with $M_{\mathrm{w}}=3.9$ ). During the post-activity phase, the sequence displays a lower $b$-value of 0.8 and a higher $\alpha \sim 1.5$, which respectively point to a lower proportion of small magnitude events and more aftershock productivity. This change can indicate a possible transition to some higher stress level due to a continuous aseismic forcing since the pre-activity period.

$\boldsymbol{\nabla}$ In Yazdi et al. (2018), we study the 2012 Ahar-Varzeghan earthquake sequence (chapter 4). We analyse a series of aftershocks that are not associated to a single mainshock, but to a doublet of $M_{\mathrm{w}} 6.4$ and $M_{\mathrm{w}} 6.2$ earthquakes with a very close spatiotemporal separation. The result of temporal ETAS modelling with cut-off magnitude of 2.0, shows that the aftershock proportion is relatively small and about $30 \%$ (with $\alpha=1.48$ ). In turn, applying bigger cut-off magnitudes of 2.5 and 3.0 results in an increase in the aftershock productivity parameter $\alpha$, to 1.67 and 1.88 , respectively. This implicates that the earthquake-earthquake triggering constructs the majority of the earthquake population (Figure 5 in chapter 4) with magnitudes $\geq 2.5$.

The GR modelling shows that the $b$-value does not vary significantly during the sequence and stays below 1.0 (Figure 4 in chapter 4 ), showing a considerable contribution of larger magnitudes.

Overall, the incomplete catalog records of low-magnitude events for this sequence, and our choice for a relatively high cut-off magnitude of 2.0, have a considerable influence on the result of ETAS modelling. Accordingly, the less energetic lowmagnitude events are mainly associated with the background activity. Some authors (e.g., Djamour et al., 2011; Ghods et al., 2015) indicate that despite the absence of important earthquake activity during the recent past, tectonic stresses are actively accumulating in the study area. Our results support that in addition to the elastic stress release due to the mainshock-aftershock mechanism and the build-up tectonic stress on the faults, an ongoing aseismic forcing with yet-undefined source contributes to the generation of background earthquakes with small magnitudes. 
$\boldsymbol{\nabla}$ In addition to the two crustal earthquake sequences mentioned above, in Yazdi et al. (2019) we apply the ETAS model to the subduction interface earthquake activity during almost two decades in the area where the 2014 Papanoa (Mexico) earthquake sequence occurred (chapter 6). The observed sudden increase in the overall population of earthquakes since the Papanoa mainshock with $M_{\mathrm{w}} 7.3$ was modelled and the variations of activity rate $\lambda\left(t \mid \mathcal{H}_{t}\right)$ were analysed.

For a relatively high $m_{c}=3.8$, we found a very small fluctuation in the background rate $\mu(t)$, where it mostly stays around $0.011 d^{-1}$, and then it jumps up to $0.017 d^{-1}$ following this large thrust earthquake. However, the $m_{c}$ since 2014 has a lower value of 2.9 for which $\mu(t)$ remains constant again (Figure 7.1d).

The achieved results point to a long term or lasting (at least until the end of 2017) rise in the state of aseismic forcing following the Papanoa mainshock. Apparently, the subduction interface in this area is experiencing a higher contribution of tectonic earthquakes with magnitudes $\geq 3.8$ in comparison to the pre-mainshock period. Table 2 in chapter 6 shows that $\alpha$-value does not exhibit a considerable aftershock productivity as it is usually expected for a large thrust earthquake (Hainzl et al., 2010). We also attempt to apply the spatiotemporal version of ETAS modelling with constant background rate $\mu$ (Lombardi, 2017). The obtained difference between the spatial distribution of the background activity before and after the Papanoa mainshock is consistent with the location of the mainshock and two other large events with $M_{\mathrm{w}} 6.6$ and $M_{\mathrm{w}} 6.2$ on $8^{\text {th }}$ and $10^{\text {th }}$ May 2014 , respectively.

Other critical parameter in statistical analysis of aftershock sequence is the power of the aftershock rate decay or $p$. We have seen that the $p$ value stays close to 1.1 (tables 2 in both chapters 4 and 6) for both mainshock-aftershock sequences, which is perfectly within the given range $[0.8,1.2]$ by Utsu \& Ogata (1995).

In summary, the results of applying ETAS modelling on these mainshock-aftershock sequences, does not represent a high aftershock population due to earthquake-earthquake triggering as it is expected for large magnitude mainshocks. Hainzl et al. (2013) discuss that the magnitudedependent aftershock productivity (or $\alpha$ ) can be underestimated in the ETAS modelling if the catalog limitations and aseismic transients are ignored. In modellings presented above, the aseismic transients are not neglected in the parameter estimations, as we consider time-dependent background rates. In turn, missing small magnitude records in our catalogs influences the relatively low productivity values for $\alpha$ that would be compensated, via the process of parameter optimization, by abrupt increases in the background rate $\mu(t)$. Indeed, overcoming the deficiency of the earthquake database, notwithstanding the statistical model we use, can lead to more definitive conclusions and further implications, as for the seismic hazard and risk.

I also apply the physics-based approach in this dissertation. This approach is implemented by computing the changes in Coulomb failure stress ( $\triangle \mathrm{CFS}$ ) following a slip over a rupturing surface which can be a fault plane or subducting interface. For $\triangle$ CFS modelling, it is essential to have a reliable rupture model, which gives the slip distribution over the ruptured surface. Moreover, an improved accuracy in the determination of aftershocks hypocentral location is 
crucial for interpreting a cause-effect relation between mainshock and aftershocks. A summary of the achieved conclusions is listed below:

$\boldsymbol{\nabla}$ In Yazdi et al. (2018), we survey the triggering impact of the first mainshock on the occurrence of the second one for which no clear evidence about its rupture plane orientation was previously found. We design a multiple-strike rupture plane for the first shock with $M_{\mathrm{w}} 6.4$ and compute its triggering impact on the second shock with $M_{\mathrm{w}} 6.2$, at its hypocentral location (Ghods et al., 2015), using the focal mechanism solutions given by CMT. We also analyse the spatial relation of the 2012 Ahar-Varzeghan doublet and its aftershocks distribution, which exhibits a mainly EW epicentral alignment, though the largest aftershock with $M_{\mathrm{w}} 5.6$ on $7^{\text {th }}$ November, is accompanied by a NS orientated cluster.

The result suggests that if the first shock triggered the second one, it is more likely that the second shock occurred along an almost perpendicular geometry $\left(10^{\circ} / 50^{\circ} / 36^{\circ}\right)$ to the first shock. This result supports the interpretation of some other studies such as Donner et al. (2015), and contradicts the findings of Ansari (2016) and Momeni \& Tatar (2018) about the rupturing plane for the second shock.

Applying the obtained result about the focal mechanism of the doublet, we compute $\triangle$ CFS maps for optimum thrust and strike-slip ruptures in the area. To reduce the adverse influence of high depth uncertainties, the $\triangle$ CFS maps are estimated for two depth ranges of $5 \mathrm{~km}$ thickness $([4,9] \mathrm{km}$ and $[16,21] \mathrm{km})$ and the averaged value in each range is compared with the spatial distribution of relocated aftershocks within that depth range. The result exhibits considerable consistency of the positively charged area with the aftershock distribution for the depth range [16, 21] $\mathrm{km}$ and both optimum thrust and strike-slip mechanisms. However, the shallow cluster of the relocated aftershocks does not present a significant coincidence with the positively charged areas for none of the rupture mechanisms.

$\boldsymbol{\nabla}$ In Yazdi et al. (2019), we analyse the consistency of aftershock distribution with the positive $\triangle$ CFS pattern due to the Papanoa earthquake with $M_{\mathrm{w}} 7.3$ and the next two largest events of the sequence with $M_{\mathrm{w}} 6.6$ and 6.2. The $\Delta \mathrm{CFS}$ is estimated for pure thrust slip over the subduction interface. To account for depth errors of the aftershock hypocenter, we consider earthquakes with $\pm 5 \mathrm{~km}$ vertical distance to the subduction interface with a thickness of $10 \mathrm{~km}$. The result shows that the $\triangle \mathrm{CFS}$ map computed using the slip model by Mendoza \& Martínez López (2017) highly agrees with the distribution of relocated aftershocks.

$\boldsymbol{\nabla}$ Chapter 5 presents another application of $\triangle \mathrm{CFS}$ calculation, which I use for investigating the causality of mainshock-aftershock earthquake sequence of the 2001 Coyuca in the Guerrero seismic gap. In this study, I program a 3D slip model over an expanded area of Guerrero subduction zone in Mexico. This model uses the short-term coupling index between the Cocos and the North American plates that is estimated using GPS inversions for inter-SSE periods of $\sim 4$ years by Radiguet et al. (2016). I consider a constant velocity for the Cocos plate shortening along the 
middle American trench and simulate the back-slip over patches of $5 \times 5 \mathrm{~km}^{2}$ based on a $3 \mathrm{D}$ model of coupling index. The $\triangle \mathrm{CFS}$ maps due to the subduction interface back-slip model do not show a clear triggering influence of 1998-2001 inter-SSE on the occurrence of $8^{\text {th }}$ October 2001 Coyuca mainshock. The result indicates a small positive $\Delta \mathrm{CFS} \sim+0.005$ bars on the shallower depth of $5 \mathrm{~km}$, on a southward rupture plane given by Iglesias et al. (2004). However, at higher depth $\Delta \mathrm{CFS} \sim-0.1$ bars. On the contrary, applying only 10\% of slip during 2001-2002 SSE can cause a positive $\triangle \mathrm{CFS}$ up to +0.05 bars on the hypocentral area of the Coyuca series, and the hypocentral locations of aftershocks highly coincide with the positively charged areas. A combination of 1998-2001 inter-SSE back-slip and 2001-2002 SSE total slip modelling does not show a significant change in the inconclusive result from back-slip modelling. Nevertheless, considering the existence of a southward normal fault, which was very close to rupture since long before, the triggering influence of early SSE phase is not unlikely. Specially, because the continuation of the 2001-2002 SSE expands the positive $\triangle \mathrm{CFS}$ area and coincides with the hypocentral distribution of aftershocks.

Yet, it is worth remarking that in all three sequences in chapters 4, 5 and 6, the relocated hypocentres comprise a fraction of the whole aftershock activity and the available data we have contain considerable depth uncertainties. Moreover, on the grounds that there are not enough focal mechanism solutions for the aftershock earthquakes, we assume that their focal mechanism share similar rupture slip directions. However, studies have shown that for crustal earthquakes (e.g., 2012 Ahar-Varzeghan and 2001 Coyuca earthquake sequences), many of the aftershocks might have dissimilar mechanisms (e.g., Santoyo et al., 2005). Overcoming the above defects would lead to much more accurate and strong conclusions.

In the chapter 6, the implication of spatiotemporal variation in the long term rate of earthquake activity for the PSHA in is analysed as below:

$\boldsymbol{\nabla}$ In Yazdi et al. (2019), we apply the result from our statistical analysis (in the time domain), which exhibits an increase in the rate of background earthquakes. Thus, we use spatiotemporal ETAS modelling for estimation of the spatial probability distribution of the background earthquakes since January 1992 up to (I) April 2014 and (II) January 2018. The achieved spatial probability distributions are applied for extracting the background density rate from the smoothed density rate of the total observed activity in subduction interface (for both periods I and II). The outputs are used as spatial distributions (in time unit) of de-clustered earthquake data in periods I and II, to compute the exceedance probability of the strong ground motion $\psi^{*}$, in one year. Similar calculations are carried out for density rate of the total observed activity (as clustered earthquake data) too.

Applying a minimum magnitude of $M_{\mathrm{w}} 5.0$, the PSHA results show that for background density rate, the changes in the ground motion exceedance rates are too small and not really significant. Whereas, using total observed density rate, we see that nearby the epicentre of Papanoa mainshock on $18^{\text {th }}$ April 2014, the peak ground 
acceleration (PGA) rises up to $7 \%$ to $0.5 \mathrm{~g}$ for annual exceedance probability of $0.2 \%$, and prompts the importance of implementing updated earthquake rates and influence of clustered data in PSHA.

Overall, the researches summarized above make an endeavor to shed more light on characterizing earthquakes with different sources and tackle a reliable earthquake rate modelling in time and space domains. Future studies can extend the implication of earthquake sequences for the PSHA further. With respect to the study in Yazdi et al. (2017), although the magnitude range of this swarm sequence is small, a time-dependent PSHA study would help updating the exceedance probabilities of the ground motion in short-term and near-field. Thus, we can consider the estimation of exceedance rate $\Lambda$, for smaller time frames of approximately constant earthquake rates, and monitor the changes in the PSHA (e.g., Convertito et al., 2012). Likewise, the ETAS modellings carried out in Yazdi et al. $(2018,2019)$ provide updated earthquake rates associated to background and aftershock activities, which construct part of the source component for future updates in PSHA studies for north-western Iran and central coast of the Guerrero, and would imply modification of PSHA in long-term. Moreover, one of the interesting questions that later could be addressed in the $\triangle \mathrm{CFS}$ modelling is about the influence of coseismic stress changes in the spatiotemporal distribution of earthquake rate. Some studies introduce models that combine other physics-based approaches like rate- and state-dependent friction laws and $\triangle \mathrm{CFS}$ to compute the spatiotemporal variations in the earthquakes rates during a period of sequential events which can produce considerable coseismic stress changes (e.g., Dieterich \& Conrad, 1984; Catalli et al., 2008; Chan et al., 2010). The result of such models would have direct implication for time-dependent PSHA studies and should be considered to explore in future researches. Ultimately, for providing more applicable PSHA results, the hybrid models that count for different time horizons should be taken into account (e.g., Rhoades, 2013; Gerstenberger et al., 2016).

I hope this effort, despite all the shortcomings, will trigger future research in the analysis of earthquake sequences and their influence on seismic hazard assessments in short-term to long-terms. 



\section{References}

Aki, K. (1966). Generation and propagation of $G$ waves from the Niigata earthquake of June 16, 1964. Part 1. A statistical analysis. Bull. Earthq. Res. Inst., 44, 23-72.

Anderson, E. M. (1905). The dynamics of faulting. Transactions of the Edinburgh Geological Society, 8(3), 387-402. doi: 10.1144/transed.8.3.387

Ansari, S. (2016). Co-seismic stress transfer and magnitude-frequency distribution due to the 2012 Varzaqan-Ahar earthquake doublets (Mw 6.5 and 6.4), NW Iran. J. Asian Earth Sci., $132,129-137$.

Anta-Sánchez, F. (2017). Análisis de la serie sísmica de Coyuca (México, 2001). Trabajo Fin de Grado, E.T.S.I. en Topografía, Geodesia y Cartografía (UPM), Madrid, Spain. Retrieved from http://oa.upm.es/47874/

Arroyo, D., García, D., Ordaz, M., Mora, M. A., \& Singh, S. K. (2010). Strong ground-motion relations for Mexican interplate earthquakes. J. Seismol., 14(4), 769-785.

Båth, M. (1965). Lateral inhomogeneities of the upper mantle. Tectonophysics, 2(6), 483-514.

Benito, B., \& Gaspar-Escribano, J. M. (2007). Ground motion characterization and seismic hazard assessment in Spain: Context, problems and recent developments. J. Seismol., 11(4), $433-452$.

Bommer, J. J., Stafford, P. J., Alarcón, J. E., \& Akkar, S. (2007). The influence of magnitude range on empirical ground-motion prediction. Bull. Seismol. Soc. Am., 97(6), 2152-2170.

Cantavella, J., Morales, J., \& Martinez-Solares, J. (2013). La serie sísmica de la comarca de La Loma (Jaén). In Informe del grupo de trabajo interinstitucional sobre la actividad sísmica en la comarca de la Loma (Jaén): Antecedentes, distribución temporal, localización y mecanismo focal, Jaén (pp. 25-38). Ministerio de Fomento, Madrid.

Carmona, E., Stich, D., Ibañez, J. M., \& Saccorotti, G. (2009). Multiplet focal mechanisms from polarities and relative locations: The Iznajar swarm in southern Spain. Bull. Seismol. Soc. Am., 99(6), 3421-3429. 
Catalli, F., Cocco, M., Console, R., \& Chiaraluce, L. (2008). Modeling seismicity rate changes during the 1997 Umbria-Marche sequence (central Italy) through a rate-and state-dependent model. J. Geophys. Res.: Solid Earth, 113(B11).

Chan, C.-H., Sørensen, M. B., Stromeyer, D., Grünthal, G., Heidbach, O., Hakimhashemi, A., \& Catalli, F. (2010). Forecasting Italian seismicity through a spatio-temporal physical model: Importance of considering time-dependency and reliability of the forecast. Ann. Geophys, 53(3), 129-140.

Chouet, B. A. (1996). Long-period volcano seismicity: Its source and use in eruption forecasting. Nature, 380(6572), 309.

Console, R., Murru, M., \& Lombardi, A. M. (2003). Refining earthquake clustering models. J. Geophys. Res.: Solid Earth, 108(B10).

Converse, G. (1973). Equations for the displacements and displacement derivatives due to a rectangular dislocation in a three-dimensional elastic half-space. User's Manual For DIS3D, $U, 119-148$

Convertito, V., Maercklin, N., Sharma, N., \& Zollo, A. (2012). From induced seismicity to direct time-dependent seismic hazard. Bull. Seismol. Soc. Am., 102(6), 2563-2573.

Convertito, V., \& Zollo, A. (2011). Assessment of pre-crisis and syn-crisis seismic hazard at campi flegrei and mt. vesuvius volcanoes, campania, southern italy. Bull. Volcanol., 73(6), 767-783.

Copley, A., Faridi, M., Ghorashi, M., Hollingsworth, J., Jackson, J., Nazari, H., Oveisi, B., \& Talebian, M. (2013). The 2012 August 11 Ahar earthquakes: Consequences for tectonics and earthquake hazard in the Turkish-Iranian Plateau. Geophys. J. Int., 196(1), 15-21.

Cornell, C. A. (1968). Engineering seismic risk analysis. Bull. Seismol. Soc. Am., 58(5), 1583-1606.

Crespo Álvarez, M. J., Martínez Cutillas, F. J., \& Marti Rodriguez, J. (2013). Seismic hazards of the Iberian Peninsula-evaluation with Kernel functions. Nat. Hazard Earth Sys.(1), 37633811 .

de Arcangelis, L., Godano, C., Grasso, J. R., \& Lippiello, E. (2016). Statistical physics approach to earthquake occurrence and forecasting. Phys. Rep., 628, 1-91.

Deichmann, N., \& Garcia-Fernandez, M. (1992). Rupture geometry from high-precision relative hypocentre locations of microearthquake clusters. Geophys. J. Int., 110(3), 501-517.

DeMets, C., Gordon, R. G., \& Argus, D. F. (2010). Geologically current plate motions. Geophys. J. Int., 181(1), 1-80.

Dieterich, J. H., \& Conrad, G. (1984). Effect of humidity on time-and velocity-dependent friction in rocks. J. Geophys. Res.: Solid Earth, 89(B6), 4196-4202. 
Djamour, Y., Vernant, P., Nankali, H. R., \& Tavakoli, F. (2011). NW Iran-eastern Turkey present-day kinematics: Results from the Iranian permanent GPS network. Earth Planet Sci. Lett., 307(1-2), 27-34.

Donner, S., Ghods, A., Krüger, F., Rößler, D., Landgraf, A., \& Ballato, P. (2015). The AharVarzeghan Earthquake Doublet (M w 6.4 and 6.2) of 11 August 2012: Regional Seismic Moment Tensors and a Seismotectonic Interpretation. Bull. Seismol. Soc. Am., 105(2A), 791-807.

Erickson, L. (1987). User's manual for DIS3D: A three-dimensional dislocation program with applications to faulting in the Earth, Geomechanics. Applied Earth Science Department, Stanford University, Stanford, California.

Fasola, S. L., Brudzinski, M. R., Holtkamp, S. G., Graham, S. E., \& Cabral-Cano, E. (2019). Earthquake swarms and slow slip on a sliver fault in the Mexican subduction zone. P. Natl. Acad. Sci. USA, 116(15), 7198-7206. doi: 10.1073/pnas.1814205116

Felzer, K. R., Abercrombie, R. E., \& Ekström, G. (2004). A common origin for aftershocks, foreshocks, and multiplets. Bull. Seismol. Soc. Am., 94(1), 88-98.

Franco, S. I., Kostoglodov, V., KM, L., Manea, M., \& JA, S. (2005). Propagation of the 2001-2002 silent earthquake and interplate coupling in the Oaxaca subduction zone, Mexico. Earth Planets Space, 57(10), 973-985.

Gerstenberger, M. C., Rhoades, D. A., \& McVerry, G. H. (2016). A hybrid time-dependent probabilistic seismic-hazard model for Canterbury, New Zealand. Seismol. Res. Lett., 87(6), 1311-1318.

Ghods, A., Shabanian, E., Bergman, E., Faridi, M., Donner, S., Mortezanejad, G., \& Aziz-Zanjani, A. (2015). The Varzaghan-Ahar, Iran, earthquake doublet (Mw 6.4, 6.2): Implications for the geodynamics of northwest Iran. Geophys. J. Int., 203(1), 522-540.

Global Centroid Moment Tensor. (2019). https: / /www.globalcmt.org/CMTsearch . html. (last accessed; October 2019)

Got, J. L., Fréchet, J., \& Klein, F. W. (1994). Deep fault plane geometry inferred from multiplet relative relocation beneath the south flank of Kilauea. J. Geophys. Res.: Solid Earth, 99(B8), $15375-15386$.

Graham, S., DeMets, C., Cabral-Cano, E., Kostoglodov, V., Rousset, B., Walpersdorf, A., Cotte, N., Lasserre, C., McKaffrey, R., \& Salazar-Tlaczani, L. (2015). Slow slip history for the Mexico subduction zone: 2005 through 2011. In Geodynamics of the Latin American Pacific margin (pp. 3445-3465). Springer.

Gutenberg, B., \& Richter, C. F. (1944). Frequency of earthquakes in California. Bull. Seismol. Soc. Am., 34(4), 185-188.

Hainzl, S., Brietzke, G. B., \& Zöller, G. (2010). Quantitative earthquake forecasts resulting from static stress triggering. J. Geophys. Res.: Solid Earth, 115(B11). 
Hainzl, S., \& Fischer, T. (2002). Indications for a successively triggered rupture growth underlying the 2000 earthquake swarm in Vogtland/NW Bohemia. J. Geophys. Res.: Solid Earth, 107(B12), ESE-5.

Hainzl, S., \& Marsan, D. (2008). Dependence of the Omori-Utsu law parameters on main shock magnitude: Observations and modeling. J. Geophys. Res.: Solid Earth, 113(B10). doi: 10.1029/2007JB005492

Hainzl, S., \& Ogata, Y. (2005). Detecting fluid signals in seismicity data through statistical earthquake modeling. J. Geophys. Res.: Solid Earth, 110(B5). doi: 10.1029/2004JB003247

Hainzl, S., Zakharova, O., \& Marsan, D. (2013). Impact of aseismic transients on the estimation of aftershock productivity parameters. Bull. Seismol. Soc. Am., 103(3), 1723-1732.

Hanks, T. C., \& Kanamori, H. (1979). A moment magnitude scale. J. Geophys. Res.:Solid Earth, 84(B5), 2348-2350.

Harris, R. A. (1998). Introduction to special section: Stress triggers, stress shadows, and implications for seismic hazard. J. Geophys. Res.: Solid Earth, 103(B10), 24347-24358.

Harris, R. A. (2000). Earthquake stress triggers, stress shadows, and seismic hazard. Curr Sci INDIA, 79(9), 1215-1225.

Harris, R. A. (2017). Large earthquakes and creeping faults. Rev. Geophys., 55(1), 169-198.

Heidbach, O., Rajabi, M., Cui, X., Fuchs, K., Müller, B., Reinecker, J., Reiter, K., Tingay, M., Wenzel, F., \& Xie, F. (2018). The World Stress Map database release 2016: Crustal stress pattern across scales. Tectonophysics, 744, 484-498. doi: 10.1016/j.tecto.2018.07.007

Heidbach, O., Rajabi, M., Reiter, K., \& Ziegler, M. (2016). WSM Team (2016): World Stress Map Database Release 2016. GFZ Data Services. doi: 10.5880/WSM.2016.001

Helmstetter, A., \& Sornette, D. (2002). Subcritical and supercritical regimes in epidemic models of earthquake aftershocks. J. Geophys. Res.: Solid Earth, 107(B10), ESE-10.

Hill, D. P. (1977). A model for earthquake swarms. J. Geophys. Res., 82(8), 1347-1352.

Husen, S., \& Hardebeck, J. (2010). Earthquake location accuracy, community online resource for statistical seismicity analysis. Community Online Resource for Statistical Seismicity Analysis. Retrieved from http: / / www. corssa.org doi: 10.5078/corssa-55815573

Huxley, T. H. (1869). Nature: Aphorisms by Goethe. Nature, 1, 9-11.

Ide, S. (2007). Slip inversion. In Treatise on geophysics: Earthquake seismology (volume editor:H. Kanamori) (Vol. 4, pp. 193-224). Elsevier.

Iglesias, A., Clayton, R., Pérez-Campos, X., Singh, S., Pacheco, J., García, D., \& ValdésGonzález, C. (2010). S wave velocity structure below central México using high-resolution surface wave tomography. J. Geophys. Res.: Solid Earth, 115(B6). doi: 10.1029/2009JB006332 
Iglesias, A., Pacheco, J. F., \& Singh, S. K. (2004). El sismo de Coyuca del 8 de octubre del 2001 $(\mathrm{Mw}=5.8)$ : Una falla normal sobre la brancha sísmics de Guerrero. UNAM, Mexcio.

IGN, \& UPM. (2013). Actualización de mapas de peligrosidad sísmica de España 2012, isbn:978-84-416-2685-0.

Jackson, J., \& White, N. (1989). Normal faulting in the upper continental crust: Observations from regions of active extension. J. Struct. Geol., 11(1-2), 15-36.

Ji, C., Wald, D. J., \& Helmberger, D. V. (2002). Source description of the 1999 Hector Mine, California, earthquake, part I: Wavelet domain inversion theory and resolution analysis. Bull. Seismol. Soc. Am., 92(4), 1192-1207.

Kagan, Y. Y. (1991). Likelihood analysis of earthquake catalogues. Geophys. J. Int., 106(1), $135-148$.

Kagan, Y. Y., \& Jackson, D. D. (1995). New seismic gap hypothesis: Five years after. J. Geophys. Res.: Solid Earth, 100(B3), 3943-3959.

Kagan, Y. Y., \& Jackson, D. D. (1999). Worldwide doublets of large shallow earthquakes. Bull. Seismol. Soc. Am., 89(5), 1147-1155.

Kanamori, H. (1970). The Alaska earthquake of 1964: Radiation of long-period surface waves and source mechanism. J. Geophys. Res., 75(26), 5029-5040.

Kennet, B. (1991). IASPEI 1991 seismological tables. Terra Nova, 3(2), 122-122.

Kijko, A. (2004). Estimation of the maximum earthquake magnitude, M max. Pure Appl. Geophys., 161(8), 1655-1681.

King, G. C. P., \& Cocco, M. (2001). Fault interaction by elastic stress changes: New clues from earthquake sequences. Advances in Geophysics, 44, 1-VIII. doi: 10.1016/S0065-2687(00) 80006-0

King, G. C. P., Stein, R. S., \& Lin, J. (1994). Static stress changes and the triggering of earthquakes. Bull. Seismol. Soc. Am., 84(3), 935-953.

Kostoglodov, V., Bandy, W., Dominguez, J., \& Mena, M. (1996). Gravity and seismicity over the Guerrero seismic gap, Mexico. Geophys. Res. Lett, 23(23), 3385-3388.

Kostoglodov, V., Singh, S. K., Santiago, J. A., Franco, S. I., Larson, K. M., Lowry, A. R., \& Bilham, R. (2003). A large silent earthquake in the Guerrero seismic gap, Mexico. Geophys. Res. Lett., 30(15).

Kramer, S. L. (1996). Geotechnical earthquake engineering. Prentice Hall, New Jersey.

Lay, T., \& Wallace, T. C. (1995). Modern global seismology (Vol. 58). Elsevier, San Diego, California.

Lohman, R. B., \& McGuire, J. J. (2007). Earthquake swarms driven by aseismic creep in the Salton Trough, California. J. Geophys. Res.: Solid Earth, 112(B4). 
Lombardi, A. M. (2017). SEDA: A software package for the Statistical Earthquake Data Analysis. Scientific Reports, 7, 44171.

Mai, P. M., \& Thingbaijam, K. (2014). SRCMOD: An online database of finite-fault rupture models. Seismol. Res. Lett., 85(6), 1348-1357.

Marsan, D., Prono, E., \& Helmstetter, A. (2013). Monitoring aseismic forcing in fault zones using earthquake time series. Bull. Seismol. Soc. Am., 103(1), 169-179.

Martínez-Díaz, J., Rodríguez-Escudero, E., Álvarez-Gómez, J., Insua-Arévalo, J., \& Capote del Villar, R. (2014). Tectonic setting of the recent damaging seismic series in the Southeastern Betic Cordillera, Spain. Bull. Earthq. Eng., 12(5).

Martin Mai, P., Shearer, P., Ampuero, J. P., \& Lay, T. (2016). Standards for documenting finite-fault earthquake rupture models. Seismol. Res. Lett., 87(3), 712-718.

McNutt, S. R. (2005). Volcanic Seismology. Annu. Rev. Earth Planet. Sci., 33(1), 461-491. doi: 10.1146/annurev.earth.33.092203.122459

Mendoza, C., \& Martínez López, M. R. (2017). The Mw 7.3 Papanoa, México earthquake of April 18, 2014: Implications for recurrent $M \geq 7$ thrust earthquakes in western Guerrero. Geofis. Int., 56(1), 13-26.

Mignan, A. (2011). Retrospective on the accelerating seismic release (ASR) hypothesis: Controversy and new horizons. Tectonophysics, 505(1-4), 1-16.

Mignan, A., \& Woessner, J. (2012). Estimating the magnitude of completeness for earthquake catalogs. Retrieved from http://www. corssa.org doi: 10.5078/corssa-00180805

Molnar, P., Anderson, R. S., \& Anderson, S. P. (2007). Tectonics, fracturing of rock, and erosion. J. Geophys. Res.: Earth Surface, 112(F3).

Momeni, S., \& Tatar, M. (2018). Mainshocks/aftershocks study of the August 2012 earthquake doublet on Ahar-Varzaghan complex fault system (NW Iran). Phys. Earth Planet. In., 283, $67-81$.

Morales, J., Azañón, J., Stich, D., Roldán, F., Pérez-Peña, J., Martín, R., Cantavella, J., Martín, J., Mancilla, F., \& González-Ramón, A. (2015). The 2012-2013 earthquake swarm in the eastern Guadalquivir basin (South Spain): A case of heterogeneous faulting due to oroclinal bending. Gondwana Research, 28(4), 1566-1578. doi: 10.1016/j.gr.2014.10.017

Morgan, W. J. (1971). Convection plumes in the lower mantle. Nature, 230(5288), 42.

Musmeci, F., \& Vere-Jones, D. (1992). A space-time clustering model for historical earthquakes. Annu. Inst. Stat. Math., 44(1), 1-11.

Nishenko, S., \& Singh, S. (1987). Conditional probabilities for the recurrence of large and great interplate earthquakes along the Mexican subduction zone. Bull. Seismol. Soc. Am., 77(6), 2095-2114. 
Nur, A., \& Booker, J. R. (1972). Aftershocks caused by pore fluid flow? Science, 175(4024), $885-887$.

Nuttli, O. W. (1983). Average seismic source-parameter relations for mid-plate earthquakes. Bull. Seismol. Soc. Am., 73(2), 519-535.

Ogata, Y. (1988). Statistical models for earthquake occurrences and residual analysis for point processes. J. Am. Stat. Assoc., 83(401), 9-27.

Ogata, Y. (1998). Space-time point-process models for earthquake occurrences. Annu. Inst. Stat. Math., 50(2), 379-402.

Ogata, Y. (2006). Statistical analysis of seismicity-Updated version (SASeis2006). In Computer science monographs (Vol. 33, pp. 1-28). The Institute of Statistical Mathematics Tokyo.

Ogata, Y., \& Zhuang, J. (2006). Space-time etas models and an improved extension. Tectonophysics, 413(1-2), 13-23.

Okada, Y. (1992). Internal deformation due to shear and tensile faults in a half-space. Bull. Seismol. Soc. Am., 82(2), 1018-1040.

Omori, F. (1894). On the aftershocks of earthquakes. Journal of the College of Science, Imperial University of Tokyo, 7, 111-200.

Ouillon, G., \& Sornette, D. (2005). Magnitude-dependent Omori law: Theory and empirical study. J. Geophys. Res.: Solid Earth, 110(B4).

Pacheco, J. F., \& Singh, S. K. (2010). Seismicity and state of stress in Guerrero segment of the Mexican subduction zone. J. Geophys. Res.: Solid Earth, 115(B1). doi: 10.1029/ 2009JB006332

Peláez, J. A., García-Tortosa, F. J., Sánchez-Gómez, M., de Galdeano, C. S., Pérez-Valera, F., \& Hernares-Romero, J. (2013). la serie sísmica de Torreperogil-Sabiote (Jaén). Enseñanza de las Ciencias de la Tierra, 21(3), 336.

Radiguet, M., Cotton, F., Vergnolle, M., Campillo, M., Walpersdorf, A., Cotte, N., \& Kostoglodov, V. (2012). Slow slip events and strain accumulation in the Guerrero gap, México. J. Geophys. Res.: Solid Earth, 117(B4). doi: 10.1029/2009JB006332

Radiguet, M., Perfettini, H., Cotte, N., Gualandi, A., Valette, B., Kostoglodov, V., Lhomme, T., Walpersdorf, A., Cano, E., \& Campillo, M. (2016). Triggering of the $2014 \mathrm{Mw} 7.3$ Papanoa earthquake by a slow slip event in Guerrero, México. Nat. Geoci., 9(11), 829.

Rathbun, S. (1993). Modeling marked spatio-temporal point patterns. Bull. Int. Statist. Inst., 55(2), 379-396.

Reasenberg, P. A., \& Jones, L. M. (1989). Earthquake hazard after a mainshock in California. Science, 243(4895), 1173-1176.

Reasenberg, P. A., \& Jones, L. M. (1994). Earthquake aftershocks: Update. Science, 265(5176), 1251-1253. 
Reasenberg, P. A., \& Simpson, R. W. (1992). Response of regional seismicity to the static stress change produced by the Loma Prieta earthquake. Science, 255(5052), 1687-1690.

Reid, H. F. (1910). The California earthquake of April 18, 1906: Report of the State Earthquake Investigation Commission (Vol. 2). Carnegie institution of Washington.

Reilinger, R., McClusky, S., Vernant, P., Lawrence, S., Ergintav, S., Cakmak, R., ... others (2006). GPS constraints on continental deformation in the Africa-Arabia-Eurasia continental collision zone and implications for the dynamics of plate interactions. J. Geophys. Res.: Solid Earth, 111(B5).

Rhoades, D. A. (2013). Mixture models for improved earthquake forecasting with short-tomedium time horizons. Bull. Seismol. Soc. Am., 103(4), 2203-2215.

Rice, J. R. (1992). Fault stress states, pore pressure distributions, and the weakness of the San Andreas fault. International Geophysics, 51, 475-503. doi: 10.1016/S0074-6142(08)62835-1

Rice, J. R., \& Cleary, M. P. (1976). Some basic stress diffusion solutions for fluid-saturated elastic porous media with compressible constituents. Rev. Geophys., 14(2), 227-241.

Ruiz-Barajas, S., Sharma, N., Convertito, V., Zollo, A., \& Benito, B. (2017). Temporal evolution of a seismic sequence induced by a gas injection in the eastern coast of Spain. Sci. Rep.(72901). doi: 10.1038/s41598-017-02773-2

Santoyo, M. A., Singh, S. K., Mikumo, T., \& Ordaz, M. (2005). Space-time clustering of large thrust earthquakes along the Mexican subduction zone: An evidence of source stress interaction. Bull. Seismol. Soc. Am., 95(5), 1856-1864.

Scholz, C. H. (2019). The mechanics of earthquakes and faulting. Cambridge university press.

Servicio Sismólgico Nacional. (2019). http: / / laxdoru.igeofcu . unam.mx/cmt. (last accessed; October 2019)

Shearer, P. M. (2019). Introduction to seismology. Cambridge university press.

Shimazaki, K., \& Nakata, T. (1980). Time-predictable recurrence model for large earthquakes. Geophys. Res. Lett., 7(4), 279-282.

Sibson, R. H., \& Xie, G. (1998). Dip range for intracontinental reverse fault ruptures: Truth not stranger than friction? Bull. Seismol. Soc. Am., 88(4), 1014-1022.

Silverman, B. W. (1986). Monographs on statistics and applied probability. In Density estimation for statistics and data analysis (Vol. 26). Springer.

Skempton, A. (1954). The pore-pressure coefficients A and B. Geotechnique, 4(4), 143-147.

Spence, W., Sipkin, S. A., \& Choy, G. L. (1989). Measuring the size of an earthquake. Earthquake Information Bulletin (USGS), 21(1), 58-63.

Stacey, F. D., Davis, P. M., et al. (1977). Physics of the earth (Vol. 2). Wiley New York. 
Stein, R. S. (1999). The role of stress transfer in earthquake occurrence. Nature, 402(6762), 605.

Stirling, M., Goded, T., Berryman, K., \& Litchfield, N. (2013). Selection of earthquake scaling relationships for seismic-hazard analysis. Bull. Seismol. Soc. Am., 103(6), 2993-3011.

Toda, S., Stein, R. S., Reasenberg, P. A., Dieterich, J. H., \& Yoshida, A. (1998). Stress transferred by the $1995 \mathrm{Mw}=6.9$ Kobe, Japan, shock: Effect on aftershocks and future earthquake probabilities. J. Geophys. Res.: Solid Earth, 103(B10), 24543-24565.

UNAM Seismology Group. (2015). Papanoa, México earthquake of 18 April 2014 (Mw 7.3). Geofis. Int., 54(4), 363-386.

Utsu, T. (1957). Magnitudes of earthquakes and occurrence of their aftershocks. Zisin, Ser. 2, $10,35-45$.

Utsu, T. (1971). Aftershocks and earthquake statistics (2): Further investigation of aftershocks and other earthquake sequences based on a new classification of earthquake sequences. Journal of the Faculty of Science, Hokkaido University. Series 7, Geophysics, 3(4), 197-266.

Utsu, T., \& Ogata, Y. (1995). The centenary of the Omori formula for a decay law of aftershock activity. J. Phys. Earth, 43(1), 1-33.

Vidale, J. E., \& Shearer, P. M. (2006). A survey of 71 earthquake bursts across southern California: Exploring the role of pore fluid pressure fluctuations and aseismic slip as drivers. J. Geophys. Res.: Solid Earth, 111(B5).

Wald, D. J., Quitoriano, V., Heaton, T. H., \& Kanamori, H. (1999). Relationships between peak ground acceleration, peak ground velocity, and modified Mercalli intensity in California. Earthq. Spectra, 15(3), 557-564.

Waldhauser, F., \& Ellsworth, W. L. (2000). A double-difference earthquake location algorithm: Method and application to the northern Hayward fault, California. Bull. Seismol. Soc. Am., 90(6), 1353-1368.

Weichert, D. H. (1980). Estimation of the earthquake recurrence parameters for unequal observation periods for different magnitudes. Bull. Seismol. Soc. Am., 70(4), 1337-1346.

Wells, D. L., \& Coppersmith, K. J. (1994). New empirical relationships among magnitude, rupture length, rupture width, rupture area, and surface displacement. Bull. Seismol. Soc. Am., 84(4), 974-1002.

Woo, G. (1996a). Kernel estimation methods for seismic hazard area source modeling. Bull. Seismol. Soc. Am., 86(2), 353-362.

Woo, G. (1996b). Seismic hazard program: KERFRACT. Program Documentation.

Yamashita, T. (1999). Pore creation due to fault slip in a fluid-permeated fault zone and its effect on seismicity: Generation mechanism of earthquake swarm. In Seismicity patterns, their statistical significance and physical meaning (pp. 625-647). Springer. 
Yang, X. M., \& Davis, P. M. (1986). Deformation due to a rectangular tension crack in an elastic half-space. Bull. Seismol. Soc. Am., 76(3), 865-881.

Yazdi, P., Gaspar-Escribano, J. M., Martinez-Cuevas, S., \& Chavarria-Meneces, N. A. (2018b). Web-based tool for scenario-based seismic risk assessment. Geophys. Res. Abs., 20 EGU, 1981.

Yazdi, P., Gaspar-Escribano, J. M., Santoyo, M. A., \& Staller, A. (2019). Analysis of the 2014 Mw 7.3 Papanoa (Mexico) earthquake: Implication for seismic hazard assessment. Seismol. Res. Lett., 90, 1801-1811. doi: 10.1785/0220190032

Yazdi, P., Hainzl, S., \& Gaspar-Escribano, J. M. (2017). Statistical analysis of the 2012-2013 Torreperogil-Sabiote seismic series, Spain. J. Seismol., 21(4), 705-717. doi: 10.1007/ s10950-016-9630-4

Yazdi, P., Santoyo, M. A., \& Gaspar-Escribano, J. M. (2018). Analysis of the 2012 AharVarzeghan (Iran) seismic sequence: Insights from statistical and stress transfer modeling. Global. Planet. Change., 161, 121-131. doi: 10.1016/j.gloplacha.2017.12.007

Zhang, C., Lomnitz, C., Shi, Y., \& Ma, L. (2009). Triggering of the 2001-2002 Mexico slow-slip event, mw 7.5, by the shallow normal earthquake of 8 October 2001, Mw 5.8, and its aftershocks. Seismol. Res. Lett, 80(1), 108-118.

Zhuang, J., Chang, C. P., Ogata, Y., \& Chen, Y. I. (2005). A study on the background and clustering seismicity in the Taiwan region by using point process models. J. Geophys. Res.: Solid Earth, 110(B5). doi: 10.1029/2004JB003157

Zhuang, J., Harte, D., Werner, M. J., Hainzl, S., \& Zhou, S. (2012). Basic models of seismicity: Temporal models. Retrieved from http://www.corssa.org doi: 10.5078/corssa $-79905851$

Zhuang, J., Ogata, Y., \& Vere-Jones, D. (2002). Stochastic declustering of space-time earthquake occurrences. J. Am. Stat. Assoc., 97(458), 369-380.

Zhuang, J., Werner, M. J., Hainzl, S., Harte, D., \& Zhou, S. (2011). Basic models of seismicity: Spatiotemporal models. Retrieved from http: / / www. corssa.org doi: 10.5078/corssa $-07487583$ 